\title{
Life Cycle Impact Assessment
}

Rosenbaum, Ralph K.; Hauschild, Michael Zwicky; Boulay, Anne-Marie; Fantke, Peter; Laurent, Alexis; Núñez, Montserrat; Vieira, Marisa

\section{Published in:}

Life Cycle Assessment: Theory and practice

Link to article, DOI:

10.1007/978-3-319-56475-3_10

Publication date:

2018

Document Version

Peer reviewed version

Link back to DTU Orbit

Citation (APA):

Rosenbaum, R. K., Hauschild, M. Z., Boulay, A-M., Fantke, P., Laurent, A., Núñez, M., \& Vieira, M. (2018). Life Cycle Impact Assessment. In Life Cycle Assessment: Theory and practice (pp. 167-270). Springer.

https://doi.org/10.1007/978-3-319-56475-3_10

\section{General rights}

Copyright and moral rights for the publications made accessible in the public portal are retained by the authors and/or other copyright owners and it is a condition of accessing publications that users recognise and abide by the legal requirements associated with these rights.

- Users may download and print one copy of any publication from the public portal for the purpose of private study or research.

- You may not further distribute the material or use it for any profit-making activity or commercial gain

- You may freely distribute the URL identifying the publication in the public portal

If you believe that this document breaches copyright please contact us providing details, and we will remove access to the work immediately and investigate your claim. 


\title{
Chapter 10 Life Cycle Impact Assessment
}

\author{
Ralph K. Rosenbaum, Michael Hauschild, Anne-Marie Boulay, \\ Peter Fantke, Alexis Laurent, Montserrat Núñez and Marisa Vieira
}

\begin{abstract}
This chapter is dedicated to the third phase of an LCA study, the Life Cycle Impact Assessment (LCIA) where the life cycle inventory's information on elementary flows is translated into environmental impact scores. In contrast to the three other LCA phases, LCIA is in practice largely automated by LCA software, but the underlying principles, models and factors should still be well understood by practitioners to ensure the insight that is needed for a qualified interpretation of the results. This chapter teaches the fundamentals of LCIA and opens the black box of LCIA with its characterisation models and factors to inform the reader about: (1) the main purpose and characteristics of LCIA, (2) the mandatory and optional steps of LCIA according to the ISO standard, and (3) the science and methods underlying the assessment for each environmental impact category. For each impact category, the reader is taken through (a) the underlying environmental problem, (b) the underlying environmental mechanism and its fundamental modelling principles, (c) the main anthropogenic sources causing the problem and (d) the main methods available in LCIA. An annex to this book offers a comprehensive qualitative comparison of the main elements and properties of the most widely used and also the latest LCIA methods for each impact category, to further assist the advanced practitioner to make an informed choice between LCIA methods.
\end{abstract}

R.K. Rosenbaum $(\triangle) \cdot M$. Núñez

IRSTEA, UMR ITAP, ELSA Research Group, ELSA - PACT Industrial Chair for Environmental and Social Sustainability Assessment, Montpellier, France e-mail: ralph.rosenbaum@irstea.fr

M. Hauschild · P. Fantke · A. Laurent

Division for Quantitative Sustainability Assessment, Department of Management

Engineering, Technical University of Denmark, Kongens Lyngby, Denmark

A.-M. Boulay

CIRAIG, Sherbrooke University, Sherbrooke, QC, Canada

M. Vieira

PRé Consultants bv, Amersfoort, The Netherlands

(C) Springer International Publishing AG 2018

M. Hauschild et al. (eds.), Life Cycle Assessment,

DOI 10.1007/978-3-319-56475-3_10 


\section{Learning Objectives}

After studying this chapter, the reader should be able to:

- Explain and discuss the process and main purposes of the LCIA phase of an LCA study.

- Distinguish and explain the mandatory and optional steps according to international standards for LCA.

- Differentiate and describe each of the impact categories applied in LCIA regarding:

- the underlying environmental problem.

- the environmental mechanism and its fundamental modelling principles.

- the main anthropogenic sources causing the problem.

- the main methods used in LCIA.

\subsection{Introduction}

In practice, the LCIA phase is largely automated and essentially requires the practitioner to choose an LCIA method and a few other settings for it via menus and buttons in LCA software. However, as straightforward as that may seem, without understanding a few basic, underlying principles and the meaning of the indicators, neither an informed choice of LCIA method nor a meaningful and robust interpretation of LCA results is possible. However, the important extent of science and its inherent multidisciplinarity frequently result in a perceived opacity of this phase. This chapter intends to open the black box of LCIA with its characterisation models and factors, and to accessibly explain (1) its main purpose and characteristics, (2) the mandatory and optional steps according to ISO and (3) the meaning and handling of each impact category. While this chapter is a pedagogical and focused introduction into the complex and broad aspects of LCIA, a more profound and in-depth description, targeting experienced LCA practitioners and scientists, can be found in Hauschild and Huijbregts (2015).

Once the Life Cycle Inventory is established containing all elementary flows relevant for the product system under assessment, the next question to answer will be something like: How to compare $1 \mathrm{~g}$ of lead emitted into water to $1 \mathrm{~g}$ of $\mathrm{CO}_{2}$ emitted into the air? In other words, how to compare apples with pears? Life Cycle Impact Assessment (LCIA) is a phase of LCA aiming to assess the magnitude of contribution of each elementary flow (i.e. emissions or resource use of a product system) to an impact on the environment. Its objective is to examine the product system from an environmental perspective using impact categories and category indicators in conjunction with the results of the inventory analysis. This will provide information useful in the interpretation phase.

As the focal point of this phase of an LCA (and also of this chapter), it is a relevant question to ask what is an environmental impact? It could be defined as a 
set of environmental changes, positive or negative, due to an anthropogenic intervention. Such impacts are studied and assessed using a wide range of quantitative and qualitative tools, all with specific aims and goals to inform or enable more sustainable decisions. In LCA this is an important phase, as it transforms an elementary flow from the inventory (LCI) into its potential impacts on the environment. This is necessary since elementary flows are just quantities emitted or used but not directly comparable to each other in terms of the importance of their impact. For example, $1 \mathrm{~kg}$ of methane emitted into air does not have the same impact on climate change as $1 \mathrm{~kg}$ of $\mathrm{CO}_{2}$, even though their emitted quantities are the same $(1 \mathrm{~kg})$ since methane is a much stronger greenhouse gas (GHG). LCIA characterisation methods essentially model the environmental mechanism that underlies each of the impact categories as a cause-effect chain starting from the environmental intervention (emission or physical interaction) all the way to its impact. However, the results of the LCIA should neither be interpreted as predicted actual environmental effects nor as predicted exceedance of thresholds or safety margins nor as risks to the environment or human health. The results of this LCA phase are scores that represent potential impacts, a concept that is explained further on.

The ISO 14040/14044 standards (ISO 2006a, b) distinguish mandatory and optional steps for the LCIA phase, which will all be explained further in this chapter:

\section{Mandatory steps:}

- Selection of impact categories, category indicators and characterisation models (in practice typically done by choosing an already existing LCIA method) $\rightarrow$ Which impacts do I need to assess?

- Classification (assigning LCI results to impact categories according to their known potential effects, i.e. in practice typically done automatically by LCI databases and LCA software)

$\rightarrow$ Which impact(s) does each LCI result contribute to?

- Characterisation (calculating category indicator results quantifying contributions from the inventory flows to the different impact categories, i.e. typically done automatically by LCA software)

$\rightarrow$ How much does each LCI result contribute?

Optional steps:

- Normalisation (expressing LCIA results relative to those of a reference system) $\rightarrow$ Is that much?

- Weighting (prioritising or assigning weights to the each impact category) $\rightarrow$ Is it important?

- Grouping (aggregating several impact indicator results into a group)

As already mentioned, it is important to keep in mind that the impacts that are assessed in the LCIA phase should be interpreted as impact potentials, not as actual impacts, nor as exceeding of thresholds or safety margins, or risk, because they are: 
- Relative expressions of potential impacts associated with the life cycle of a reference flow needed to support a unit of function (=functional unit)

- Based on inventory data that are integrated over space and time, and thus often occurring at different locations and over different time horizons

- Based on impact assessment data which lack information about the specific conditions of the exposed environment (e.g. the concomitant exposure to substances from other product systems)

Terminology and definitions are given in Table 10.1.

Table 10.1 Essential terminology and definitions

\begin{tabular}{|c|c|c|}
\hline Term & Definition & Source \\
\hline $\begin{array}{l}\text { Area of } \\
\text { protection }\end{array}$ & $\begin{array}{l}\text { A cluster of category endpoints of recognisable value } \\
\text { to society. Examples are human health, natural } \\
\text { resources and natural environment }\end{array}$ & $\begin{array}{l}\text { Hauschild and } \\
\text { Huijbregts (2015) }\end{array}$ \\
\hline $\begin{array}{l}\text { Category } \\
\text { indicator }\end{array}$ & Quantifiable representation of an impact category & ISO (2006b) \\
\hline $\begin{array}{l}\text { Category } \\
\text { endpoint }\end{array}$ & $\begin{array}{l}\text { Attribute or aspect of natural environment, human } \\
\text { health or resources, identifying an environmental issue } \\
\text { giving cause for concern }\end{array}$ & ISO (2006b) \\
\hline $\begin{array}{l}\text { Characterisation } \\
\text { model }\end{array}$ & $\begin{array}{l}\text { Reflect the environmental mechanism by describing } \\
\text { the relationship between the LCI results, category } \\
\text { indicators and, in some cases, category endpoint(s). } \\
\text { The characterisation model is used to derive the } \\
\text { characterisation factors }\end{array}$ & ISO (2006b) \\
\hline $\begin{array}{l}\text { Characterisation } \\
\text { factor }\end{array}$ & $\begin{array}{l}\text { Factor derived from a characterisation model which is } \\
\text { applied to convert an assigned life cycle inventory } \\
\text { analysis result to the common unit of the category } \\
\text { indicator }\end{array}$ & ISO (2006b) \\
\hline Ecosphere & $\begin{array}{l}\text { The biosphere of the earth, especially when the } \\
\text { interaction between the living and non-living } \\
\text { components is emphasised }\end{array}$ & $\begin{array}{l}\text { Oxford Dictionary } \\
\text { of English }\end{array}$ \\
\hline Elementary flow & $\begin{array}{l}\text { Material or energy entering the system being studied } \\
\text { that has been drawn from the environment without } \\
\text { previous human transformation, or material or energy } \\
\text { leaving the system being studied that is released into } \\
\text { the environment without subsequent human } \\
\text { transformation }\end{array}$ & ISO (2006b) \\
\hline $\begin{array}{l}\text { Environmental } \\
\text { impact }\end{array}$ & $\begin{array}{l}\text { Potential impact on the natural environment, human } \\
\text { health or the depletion of natural resources, caused by } \\
\text { the interventions between the technosphere and the } \\
\text { ecosphere as covered by LCA (e.g. emissions, resource } \\
\text { extraction, land use) }\end{array}$ & EC-JRC (2010a) \\
\hline $\begin{array}{l}\text { Environmental } \\
\text { mechanism }\end{array}$ & $\begin{array}{l}\text { System of physical, chemical and biological processes } \\
\text { for a given impact category, linking the life cycle } \\
\text { inventory analysis results to category indicators and to } \\
\text { category endpoints }\end{array}$ & ISO (2006b) \\
\hline
\end{tabular}


Table 10.1 (continued)

\begin{tabular}{l|l|l}
\hline Term & Definition & Source \\
\hline $\begin{array}{l}\text { Environmental } \\
\text { relevance }\end{array}$ & $\begin{array}{l}\text { Degree of linkage between category indicator result } \\
\text { and category endpoints }\end{array}$ & ISO (2006b) \\
\hline Impact category & $\begin{array}{l}\text { Class representing environmental issues of concern to } \\
\text { which life cycle inventory analysis results may be } \\
\text { assigned }\end{array}$ & ISO (2006b) \\
\hline Impact pathway & Cause-effect chain of an environmental mechanism & $\begin{array}{l}\text { Hauschild et al. } \\
\text { (2013) }\end{array}$ \\
\hline $\begin{array}{l}\text { Lidpoint method } \\
\text { indicator }\end{array}$ & $\begin{array}{l}\text { Impact category indicator located somewhere along the } \\
\text { impact pathway between emission and category } \\
\text { endpoint }\end{array}$ & $\begin{array}{l}\text { Hauschild and } \\
\text { Huijbregts (2015) }\end{array}$ \\
\hline Potential impact & $\begin{array}{l}\text { Relative performance indicators which can be the basis } \\
\text { of comparisons and optimisation of the system or } \\
\text { product }\end{array}$ & $\begin{array}{l}\text { Hauschild and } \\
\text { Huijbregts (2015) }\end{array}$ \\
\hline Technosphere & $\begin{array}{l}\text { The sphere or realm of human technological activity; } \\
\text { the technologically modified environment }\end{array}$ & $\begin{array}{l}\text { Oxford Dictionary } \\
\text { of English }\end{array}$ \\
\hline
\end{tabular}

\subsection{Mandatory Steps According to ISO 14040/14044}

\subsubsection{Selection of Impact Categories, Category Indicators and Characterisation Models}

The contents of this section have been modified from Rosenbaum, R.K.: selection of impact categories, category indicators and characterisation models in goal and scope definition, appearing as Chapter 2 of Curran MA (ed.) (2017) LCA Compendium - The Complete World of Life Cycle Assessment-Goal and scope definition in Life Cycle Assessment, Springer, Heidelberg.

The objective of selecting impact categories, category indicators and characterisation models is to find the most useful and needed ones for a given goal. To help guide the collection of information on the relevant elementary flows in the inventory analysis, the selection of impact categories must be in accordance with the goal of the study and is done in the scope definition phase prior to the collection of inventory data to ensure that the latter is targeted towards what is to be assessed in the end (see Chaps. 7 and 8 on Goal and scope definition). A frequent difficulty is determination of the criteria that define what is useful and needed in the context of the study. Some criteria are given by ISO 14044 (2006b), either as requirements or as recommendations. The requirements are obligatory for compliance with the ISO standard, and will therefore be among the focus points of a Critical Review (see Chap. 13 on Critical review). Some of these requirements and recommendations concern LCA practitioners and LCIA method developers alike, while others are most relevant for developers of LCIA methods and of LCA software. The focus is here on the former, i.e. requirements concerning LCA practitioners. 
ISO 14044 (2006b) states that the choice of impact categories needs to assure that they

- Are not redundant and do not lead to double counting

- Do not disguise significant impacts

- Are complete

- Allow traceability

Furthermore, this list is complemented with a number of obligatory criteria, requiring that the selection of impact categories, category indicators and characterisation models shall be:

- Consistent with the goal and scope of the study (when, for example, environmental sustainability assessment is the goal of a study, the practitioner cannot choose a limited set of indicators, or a single indicator footprint approach, as this would be inconsistent with the sustainability objective of avoiding burden-shifting among impact categories)

- Justified in the study report

- Comprehensive regarding environmental issues related to the product system under study (essentially meaning that all environmental issues - represented by the various impact categories - which a product system may affect need to be included, again in order to reveal any problem-shifting from one impact category to another)

- Well documented with all information and sources being referenced (in practice it is normally sufficient to provide name and version number of the LCIA method used together with at least one main reference, which should provide all primary references used to build the method)

ISO 14044 (2006b) recommendations for the selection of impact categories, category indicators and characterisation models by a practitioner include:

- International acceptance of impact categories, category indicators and characterisation models, i.e. based on an international agreement or approved by a competent international body

- Minimisation of value-choices and assumptions made during the selection of impact categories, category indicators and characterisation models

- Scientific and technical validity of the characterisation model for each category indicator (e.g. not based on unpublished or outdated material)

- Being based upon a distinct identifiable environmental mechanism and reproducible empirical observation

- Environmental relevance of category indicators

Numerous further criteria but also practical constraints beyond ISO 14044 exist and are applied, consciously or unconsciously, often based on experience or recommendations from colleagues. In practice the selection of impact categories, category indicators and characterisation models usually boils down to selecting an 
LCIA method (or several) available in the version of the LCA software that the practitioner has access to.

External factors for this choice will be among other:

- Requirements following from the defined goal (see Chap. 7) and specified in the scope definition of the LCA (see Chap. 8)

- Requirements by the commissioner of an LCA

- Fixed requirements, e.g. for Environmental Product Declarations (EPDs) or Product Environmental Footprints (PEFs) from underlying sector-based Product Category Rules (PCRs) or from labelling schemes (see Chap. 24)

Practical constraints may, for example, consist of:

- Availability, completeness and quality of LCI results required for a specific impact category

- Availability, completeness and quality of characterisation models and factors for a specific impact category, including the need to consider specific rare or new impact categories, such as noise, which may only be supported by one or two LCIA methods if at all

- If normalisation is required, availability, completeness and quality of normalisation factors for a specific impact category or LCIA method

If practical constraints prevent the practitioner from including what has been identified as relevant impact categories, this needs to be made clear in the discussion and interpretation of the LCA results and comments need to be made on whether it may change the conclusions. In the illustrative case on window frames in Chap. 39, the method recommended for characterisation by the International Life Cycle Data system (ILCD) is chosen as life cycle impact assessment method (EC-JRC 2011), and all impact categories covered by the method are included in the study.

In common LCA practice, a number of category indicators, based on specific characterisation models is combined into predefined sets or methods, often referred to as life cycle impact assessment methods or simply LCIA methods (EC-JRC 2011; Hauschild et al. 2013), available in LCA software under names such as ReCiPe, CML, TRACI, EDIP, LIME, IMPACT 2002+, etc. However, with an increasing number of LCIA methods and indicators available, the task of choosing one requires a tangible effort from the practitioner to understand the main characteristics of these methods and to keep up-to-date with the developments in the field of LCIA. A qualitative and comparative overview of the main characteristics of current LCIA methods can be found in Chap. 40 of the Annex of this book.

\subsubsection{How to Choose an LCIA Method?}

A number of LCIA methods have been published since the first one appeared in 1984. Figure 10.1 shows the most common methodologies published since 2000 


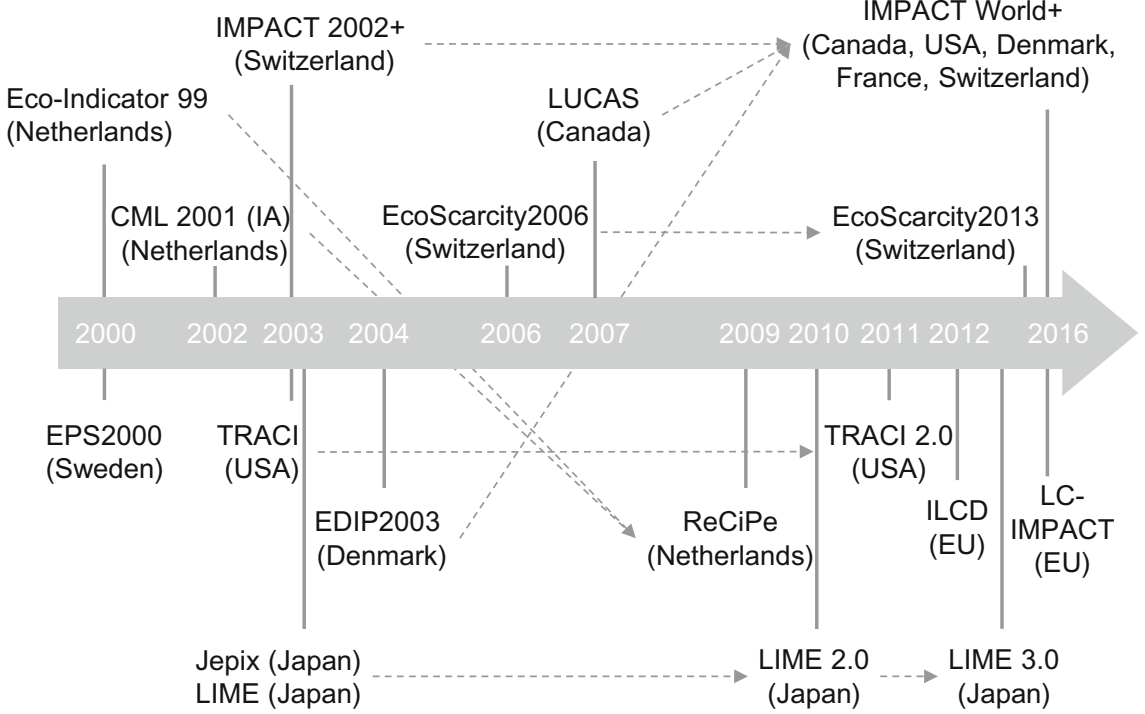

Fig. 10.1 LCIA methods published since 2000 with country/region of origin in brackets. Dotted arrows represent methodology updates (Rosenbaum 2016)

that all meet the requirements of ISO 14044. A more detailed overview of these methods can be found in Chap. 40.

When selecting an LCIA method, the requirements, recommendations, external and internal factors and constraints discussed above all need to be considered. This leads to a number of questions and criteria that should be answered in order to systematically identify the most suitable one. Here is a non-exhaustive list of relevant questions to address:

- Which impact categories (or environmental problems) do I need to cover and can I justify those that I am excluding?

- In which region does my life cycle (or its most contributing processes) take place?

- Do I need midpoint or endpoint assessment, or both?

- Which elementary flows do I need to characterise?

- Are there any recommendations from relevant organisations that can help me choose?

- How easily can the units of the impact categories be interpreted (e.g. absolute units, equivalents, monetary terms, etc.)?

- How well is the method documented?

- How easily can the results (units, aggregation into specific indicator groups, etc.) be communicated?

- Do I need to apply normalisation and if yes for which reference system (in most cases it is not recommendable to mix characterisation and normalisation factors 
from different LCIA methods due to the difference in characterisation modelling, units, numerical values, etc..)?

- When was the method published and have there been important scientific advances in the meantime?

- Do I have the resources/data availability to apply a regionalised methodology (providing more precise results)?

- Do I need to quantify the uncertainty of both LCI and LCIA and does the LCIA method support that?

ISO 14040/14044 by principle do not provide any recommendations about which LCIA method should be used, some organisations do recommend the use of a specific LCIA method or parts of it. The European Commission has established specific recommendations for midpoint and endpoint impact cátegories by systematically comparing and evaluating all relevant existing approaches per category, leading to the recommendation of the best available approach (EC-JRC 2011). This effort resulted in a set of characterisation factors, which is directly available in all major LCA software as the ILCD method. Some methods with a stronger national focus are recommended by national governmental bodies for use in their respective country, such as LIME in Japan, or TRACI in the US.

Given the amount of LCIA methods available and the amount of time required to stay informed about them, it may be tempting to essentially stick to the method(s) that the LCA practitioner knows best or has used for a long time, or that was recommended by a colleague, or simply choosing a method requested by the client to allow comparison with results from previous studies. It is however beneficial to apply a more systematic approach to LCIA method selection that in combination with the LCIA method comparison in Chap. 40 allows to determine the relevant selection questions and criteria, thus optimising the interpretability and robustness of the results of the study. The following properties are compared in Chap. 40 per impact category and for both midpoint and endpoint LCIA methods:

- Aspects/diseases/ecosystems (which kinds of impacts) that are considered

- Characterisation model used

- Selected central details about fate, exposure, effect and damage modelling

- Reliance on marginal or average indicator

- Emission compartments considered

- Time horizon considered

- Geographical region modelled

- Level of spatial differentiation considered

- Number of elementary flows covered

- Unit of the indicator

Not all of these properties may be of equal relevance for choosing an LCIA method for each practitioner or study, but they are identified here as relevant and fact-based properties. 
Further details on the selection of impact categories, category indicators and characterisation models can be found in Rosenbaum (2016) and Hauschild and Huijbregts (2015).

\subsubsection{Classification}

In this step, the elementary flows of the LCI are assigned to the impact categories to which they contribute; for example an emission of $\mathrm{CO}_{2}$ into air is assigned to climate change or the consumption of water to the water use impact category, respectively. This is not without difficulty because some of the emitted substances can have multiple impacts in two modes:

- In parallel: a substance has several simultaneous impacts, such as $\mathrm{SO}_{2}$ which causes acidification and is toxic to humans when inhaled.

- In series: a substance has an adverse effect which itself becomes the cause of something else, such as $\mathrm{SO}_{2}$ which causes acidification, which then may mobilise heavy metals in soil which are toxic to humans and ecosystems.

This step requires considerable understanding and expert knowledge of environmental impacts and is therefore typically being handled automatically by LCA software (using expert-based, pre-programmed classification tables) and not a task that the LCA practitioner needs to undertake.

\subsubsection{Characterisation}

In this step, all elementary flows in the LCI are assessed according to the degree to which they contribute to an impact. To this end, all elementary flows $E$, classified within a specific impact category $c$ (representing an environmental issue of concern), are multiplied by their respective characterisation factor CF and summed over all relevant interventions $i$ (emissions or resource extractions) resulting in an impact score IS for the environmental impact category (expressed in a specific unit equal for all elementary flows within the same impact category):

$$
\mathrm{IS}_{c}=\sum_{i}\left(\mathrm{CF}_{i} \cdot E_{i}\right)
$$

For each impact category, the indicator results are summed to determine the overall results for the category. In the following sections, the general principles of how CFs are calculated and interpreted will be discussed. In order to provide a better understanding of what CFs in each impact category represent and how they are derived, Sects. 10.6-10.16 will, for each impact category, explain the 
corresponding (1) problem observed, (2) principal environmental mechanism, (3) main causes and (4) most widely used characterisation models.

\subsubsection{What Is a Characterisation Factor?}

A characterisation factor $(\mathrm{CF})$ represents the contribution per quantity of an elementary flow to a specific environmental impact (category). It is calculated using (scientifically valid and quantitative) models of the environmental mechanism representing as realistically as possible the cause-effect chain of events leading to effects (impacts) on the environment for all elementary flows which contribute to this impact. The unit of a CF is the same for all elementary flows within an impact category. It is defined by the characterisation model developers and may express the impacts directly in absolute terms (e.g. number of disease cases/unit toxic emission) or indirectly through relating them to the impact of a reference elementary flow (e.g. $\mathrm{CO}_{2}$-equivalents/unit emission of greenhouse gases).

\subsubsection{How Is It Calculated?}

The modelling of a characterisation factor involves the use of different models and parameters and is typically conducted by experts for a particular impact category and its underlying impact pathway or environmental mechanism. Various assumptions and methodological choices are involved and this may affect the output as reflected in the differences in results that may be observed for the same impact category when applying different LCIA methods. This must be considered when interpreting the result of the LCIA phase. The first step when establishing an impact category is the observation of an adverse effect of concern in the environment, leading to the conclusion that we need to consider such effects in the context of decisions towards more sustainable developments. Once accepted as an effect of concern, the focus will be on how to characterise (quantify) the observed effect in the framework of LCA.

The basis and starting point of any characterisation model is always the establishment of a model for the environmental mechanism represented by a cause-effect chain. Its starting point is always the environmental intervention (represented by elementary flows), essentially distinguishing two types based on the direction of the relevant elementary flows between technosphere and ecosphere:

- An emission into the environment (=elementary flow from the technosphere to the ecosphere),

or

- A resource extraction from the environment (=elementary flow from the ecosphere to the technosphere). 


\subsubsection{Emission-Related Impacts}

For the first type, an emission into the environment, the principal cause-effect chain may be divided into the following main steps:

- Emission: into air, water or soil (for some product systems also other compartments may be relevant such as groundwater, indoor air, etc.)

- Fate: environmental processes causing transport, distribution and transformation of the emitted substance in the environment. Depending on the physical and chemical properties of the substance and the local conditions at the site of emission, a substance may be transferred between different environmental compartments, be transported over long distances by wind or flowing water, and be undergoing degradation and transformation into other molecules and chemical species

- Exposure: contact of the substance from the environment to a sensitive target like animals and plants, entire ecosystems (freshwater, marine, terrestrial or aerial) or humans. Exposure may involve processes like inhalation of air or ingestion of food and water

- Effects: observed adverse effects in the sensitive target after exposure to the substance, e.g. increase in the number of disease cases (ranging from reversible temporary problems to irreversible permanent problems and death) per unit intake in a human population or number of species affected (e.g. by disease, behaviour, immobility, reproduction, death, etc.) after exposure of an ecosystem

- Damage: distinguishing the severity of observed effects by quantifying the fraction of species potentially disappearing from an ecosystem, or for human health by giving more weight to death and irreversible permanent problems (e.g. reduced mobility or dysfunctional organs) than to reversible temporary problems (e.g. a skin rash or headache)

These steps together constitute the environmental mechanism of the impact category and their specific features will vary depending on the impact category we are looking at.

\subsubsection{Extraction-Related Impacts}

For the second type of elementary flow, a resource extraction from the environment, the principal cause-effect chain may comprise some or all of the following main steps (with significant simplifications possible for some resources where not all steps may be relevant, e.g. minerals):

- Extraction or use: of minerals, crude oil, water or soil, etc.

- Fate: (physical) changes to local conditions in the environment, e.g. soil organic carbon content, soil permeability, groundwater level, soil albedo, release of stored carbon, etc. 
- Exposure: change in available quantity, quality or functionality of a resource and potential competition among several users (human or ecosystems, with different degrees of ability to adapt and/or compensate), e.g. habitat loss, dehydration stress, soil biotic productivity, etc.

- Effects: adverse effects on directly affected users that are unable to adapt or compensate (e.g. diseases due to lower water quality, migration or death of species due to lack of water or habitat, malnutrition, etc.) and contributions to other impact pathways (e.g. global warming due to change in soil albedo or released soil carbon)

- Damage: distinguishing the severity of observed effects by quantifying the reduction of biodiversity, or human health of a population affected (although not yet common practice, this may even go as far as including social effects such as war on water access)

This mechanism will have specific features and may vary significantly between impact categories, but the principle remains valid for all extraction-related impact categories, currently being:

- Land Use (affecting biotic productivity, aquifer recharge, carbon sequestration, albedo, erosion, mechanical and chemical filtration capacity, biodiversity, etc.)

- Water use (affecting human health, aquatic ecosystems, terrestrial ecosystems)

- Abiotic resource use (fossil and mineral) affecting the future availability of the non-renewable abiotic resources

- Biotic resource use (e.g. fishing or wood logging) affecting the future availability of the renewable biotic resources and the ecosystems from which they are harvested.

\subsubsection{The Impact Indicator}

The starting point of the environmental mechanism is set by an environmental intervention in the form of an elementary flow in the LCI, and the contribution from the LCI flow is measured by the ability to affect an indicator for the impact category which is selected along the cause-effect chain of the impact category. Apart from the feasibility of modelling the indicator, this selection should be guided by the environmental relevance of the indicator. For example, there is limited relevance in choosing human exposure to the substance as an indicator for its human health impacts, because even if a substance is taken in by a population (i.e. exposure can be observed and quantified), it might not cause any health effect due to a low toxicity of the substance, and this would be ignored if a purely exposure-based indicator was chosen. In general, the further down the cause-effect chain an indicator is chosen, the more environmental relevance (and meaning) it will have.

However, at the same time the level of model and parameter uncertainty may increase further down the cause-effect chain, while measurability decreases (and hence the possibility to evaluate and check the result against observations that can 
be directly linked to the original cause). Contrary to a frequent misconception, that does not mean that the total uncertainty (i.e. including all its sources, not just parameter and model uncertainty) of an indicator increases when going further down the cause-effect chain, because the increase in parameter and model uncertainty is compensated by an increase in environmental relevance. If the latter is low (as is the case for indicators placed early in the cause-effect chain) the relationship of an indicator to an environmental issue is assumed but not modelled and thus hypothetical and therefore uncertain. A detailed discussion on these issues can be found in Chap. 11.

To select the impact indicator, developers must therefore strike a compromise between choosing an indicator of impact:

- Early in the environmental mechanism, giving a more measurable (e.g. in the lab) result but with less environmental relevance and more remote from the concerns directly observable in the environment

\section{Versus}

- Downstream in the environmental mechanism, giving more relevant but hardly verifiable information (e.g. degraded ecosystems, affected human lifetime)

This has led to the establishment of two different types of impact categories, applying indicators on two different levels of the environmental mechanism: midpoint impact indicators (representing option 1 from above) and endpoint impact indicators (representing option 2).

\subsubsection{Midpoint Impact Indicators}

When the impact assessment is based on midpoint impact indicators, the classification gathers the inventory results into groups of substance flows that have the ability to contribute to the same environmental effect in preparation for a more detailed assessment of potential impacts of the environmental interventions, applying the characterisation factors that have been developed for the concerned impact category. For example, all elementary flows of substances that may have a carcinogenic effect on humans will be classified in the same midpoint category called "toxic carcinogen" and the characterisation will calculate their contribution to this impact. Typical (and emerging) midpoint categories (including respective sub-categories/impact pathways) are:

- Climate change

- Stratospheric ozone depletion

- Acidification (terrestrial, freshwater)

- Eutrophication (terrestrial, freshwater, marine)

- Photochemical ozone formation

- Ecotoxicity (terrestrial, freshwater, marine)

- Human toxicity (cancer, non-cancer) 
- Particulate matter formation

- Ionising radiation (human health, aquatic and terrestrial ecosystems)

- Land Use (biotic productivity, aquifer recharge, carbon sequestration, albedo, erosion, mechanical and chemical filtration capacity, biodiversity)

- Water use (human health, aquatic ecosystems, terrestrial ecosystems, ecosystem services)

- Abiotic resource use (fossil and mineral)

- Biotic resource use (e.g. fishing or wood logging)

- Noise

- Pathogens

The characterisation at midpoint level of the elementary flows in the life cycle inventory results in a collection of midpoint impact indicator scores, jointly referred to as the characterised impact profile of the product system at midpoint level. This profile may be reported as the result of the life cycle impact assessment, and it may also serve as preparation for the characterisation of impacts at endpoint level.

\subsubsection{Endpoint Impact Indicators}

Additional modelling elements are used to expand or link midpoint indicators to one or more endpoint indicator (sometimes also referred to as damage or severity). These endpoint indicators are representative of different topics or "Areas of Protection" (AoP) that "defend" our interests as a society with regards to human health, ecosystems or planetary life support functions including ecosystem services and resources, for example. As discussed, endpoint indicators are chosen further down the cause-effect chain of the environmental mechanism closer to or at the very endpoint of the chains - the Areas of Protection. The numerous different midpoint impact categories therefore all contribute to a relatively small set of endpoint indicators as can be observed in Fig. 10.2. Although, different distinctions are possible and exist, typical endpoint impact categories are:

- Human health

- Natural environment or ecosystem quality

- Natural resources and ecosystem services

Therefore, the same list of impact categories as for midpoint indicators (see above) applies to endpoint indicators but with a further distinction regarding which of the three AoPs are affected (e.g. climate change has one midpoint indicator, but two endpoint indicators, one for human health and one for natural environmentsee Fig. 10.2). All endpoint indicators for the same AoP have a common unit and can be summed up to an aggregated impact score per AoP. Before aggregation, however, an environmental profile on endpoint level is as detailed as on midpoint level and allows for a contribution analysis of impact categories per AoP (e.g. which impact category contributes the most to human health impacts). On 


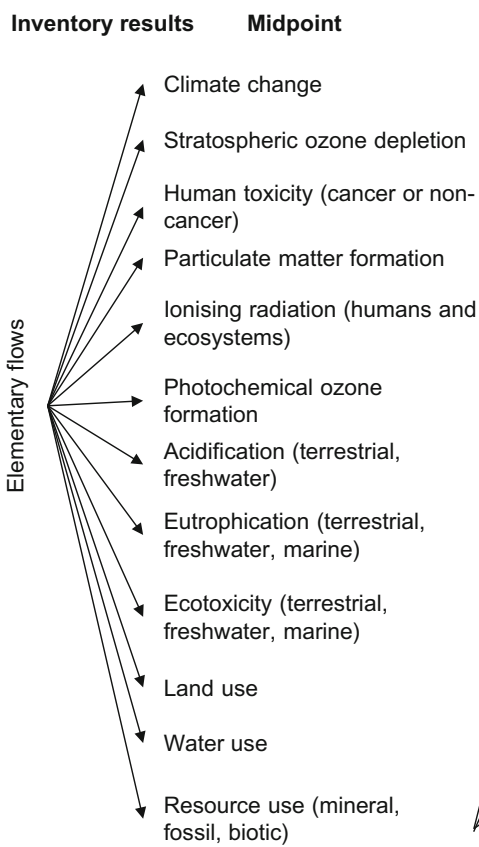

Endpoint Area of protection

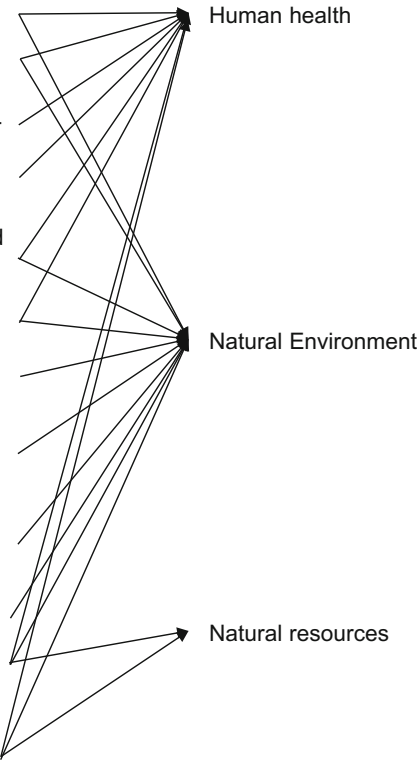

Fig. 10.2 Framework of the ILCD characterisation linking elementary flows from the inventory results to indicator results at midpoint level and endpoint level for 15 midpoint impact categories and 3 areas of protection [adapted from EC-JRC (2010b)]

midpoint level, aggregation and contribution analysis are only possible after applying normalisation and weighting, which is not needed for endpoint indicator results.

There are three frequent misconceptions related to that:

1. Misconception: Applying normalisation, weighting and aggregation to midpoint indicator results is the same as calculating endpoint indicator results. Or in other words, midpoint indicator results that are normalised, weighted and aggregated into one impact score per AoP have the same unit as endpoint indicator results aggregated into one impact score per AoP. Therefore, both results are identical. Fact: Even though the unit of both aggregated indicators is the same, their numerical value and their physical meaning are completely different. They are not identical and cannot be interpreted in the same way.

2. Misconception: Changing from midpoint-to-endpoint characterisation implies a loss of information due to aggregation from about 15 midpoints into only three endpoint indicators.

Fact: Before aggregation is applied, endpoint indicators are constituted for the same amount of impact categories as on midpoint level, but not every impact category contributes to each AoP (e.g. resource depletion does not contribute to human health impacts). Therefore, the same analysis of contribution per impact 
category is possible as for normalised and weighted midpoint indicators while avoiding the need for normalisation and weighting and the associated increased uncertainty and change in meaning.

3. Misconception: Endpoint characterisation is more uncertain than midpoint characterisation.

Fact: This may be the case when looking at a limited set of sources of uncertainty and how they contribute to the uncertainty of the value of the indicator. However, when considering all relevant sources of uncertainty and the relevance of the indicator for the decision at hand, the choice of indicator has no influence on the uncertainty of the consequences of the decision. This is discussed in detail in Chap. 11.

To go from midpoint to endpoint indicator scóres, additional midpoint-to-endpoint characterisation factors (sometimes also referred to as severity or damage characterisation factors) are needed, expressing the ability of a change in the midpoint indicator to affect the endpoint indicator. In contrast to the midpoint characterisation factors which reflect the properties of the elementary flow and hence are elementary flow-specific, the midpoint-to-endpoint characterisation factors reflect the properties of the midpoint indicator and there is hence only one per midpoint impact category. Some LCIA methods only support endpoint characterisation and here the midpoint and midpoint-to-endpoint characterisation is combined in one characterisation factor.

\subsubsection{Midpoint or Endpoint Assessment?}

Next to the relationship between environmental relevance and various sources of uncertainty discussed above (and in more detail in Chap. 11), the possibility to aggregate information from midpoint-to-endpoint level while avoiding normalisation and weighting has the advantage of providing more condensed information (fewer indicator results) to consider for a decision, while still being transparent as to which impact pathway(s) are the main causes of these damages. Instead of perceiving midpoint and endpoint characterisation as two alternatives to choose from, it is recommended to conduct an LCIA on both midpoint and endpoint level to support the interpretation of the results obtained.

\subsubsection{Time Horizons and Temporal Variability?}

Environmental impacts caused by an intervention will require different amounts of time to occur, depending on the environmental mechanism and the speed at which its processes take place. This means that next to the fact that the numerous elementary flows of an LCI may occur at different moments in time during the life cycle of the product or service assessed (which may be long for certain products like buildings for example), there is also a difference in the lag until their impacts 
occur. However, the way LCA is currently conducted, potential impacts are assessed as if interventions and potential impacts were happening instantly, aggregating them over time and over the entire life cycle. This means that these potential impacts need to be interpreted as a "backpack" of potential impacts attributable to the product or service assessed.

Next to such temporal variability, another potential source of time-related inconsistency in LCA is the problem of applying different time horizons for different impact categories. These time horizons are sometimes explicit (e.g. the 20 and 100 years' time horizons for global warming potentials), but in most cases implicit in the way the environmental mechanism has been modelled (e.g. over what time horizon the impact has been integrated). This may result in a mixing of different time horizons for different impacts in the same LCIA, which may have implications for the interpretation of LCA results. For example, methane has a lifetime much shorter than $\mathrm{CO}_{2}$. Therefore, depending on the time horizon chosen, the characterisation of methane will change. This is directly connected to the question of how to consider potential impacts affecting current and immediate future generations versus those affecting generations in a more distant future.

Another issue concerns the temporal course of the emission and its resulting impacts. While some impacts may be immediately (i.e. within a few years) tangible and directly affecting a larger number of individuals (human or not), some impacts may be very small at any given moment in time, but permanently occurring for tens to hundreds of thousands of years (e.g. impacts from heavy metal emissions from landfills or mine tailings). Between these two illustrative extremes, lies any possible combination of duration versus seyerity.

\subsubsection{Spatial Variability and Regionalisation?}

Some impacts are described as global because their environmental mechanism is the same regardless where in the world the emission occurs. Global warming and stratospheric ozone depletion are two examples. Other impacts, such as acidification, eutrophication or toxicity may be classified as regional, affecting a (sub-) continent or a smaller region surrounding the point of emission only. Impacts affecting a small area are designated as local impacts, water or direct land-use impacts on biodiversity for example. Whereas for global impact categories the site where the intervention takes place has no considerable influence on the type and magnitude of its related potential impact(s), for regional or local impacts this may influence the magnitude of the potential impact(s) up to several orders of magnitude (e.g. a toxic emission taking place in a very large and densely populated city or habitat versus somewhere remote in a large desert). This spatial variability can be dealt with in two ways:

- Identification and modelling of archetypical emission situations and their potential impacts (e.g. toxic emission into urban air, rural air or remote air) or 
spatialized archetypes (e.g. city-specific emissions, formation and background concentrations of particulate matter and related mortality rates) Or

- Modelling impacts with a certain degree of spatial resolution (e.g. sub-continental, country-level, sub-water-shed level or GPS grid-based), allowing for a characterisation which can be specific to any given place of emission

Both solutions require that the place of emission is known for each flow in the inventory-either explicitly (e.g. by country or geographical coordinates such as latitude and longitude) or regarding the most representative archetype. In order to support a spatially differentiated impact assessment, the life cycle inventory must thus not be aggregated to present one total intervention per elementary flow since this will lose the information about location of the interventions which is needed to select the right $\mathrm{CF}$. Otherwise, generic global average CFs need to be used, leading to a higher uncertainty due to the spatial variability not considered in the characterisation. In contrast to the site-generic LCIA method, which provides one CF per combination of elementary flow and intervention/emission compartment, the spatially differentiated characterisation method provides one CF per combination of elementary flow, intervention/emission compartment and spatial unit. For grid-based methods, this may amount to thousands of CFs for each contributing elementary flow.

It depends on the impact category and emission situation to evaluate whether a spatial or archetypal setup will give the more accurate solution (e.g. urban/rural differences in particulate matter-related health effects might not be captured by spatial models with typical resolutions lower than $10 \times 10 \mathrm{~km}^{2}$ at the global scale, whereas an archetypal model distinguishing between urban and rural emission situations would capture such differences). It should be noted that country-based characterisation is not meaningful from a scientific point of view, as most impacts are not influenced by political borders, although from a practical data-availability point of view this currently not unusual practice is understandable and normally an improvement to not considering the spatial variation at all. It should furthermore be noted that most currently available LCA software fails to support spatially differentiated characterisation, and therefore most LCAs are performed using the site-generic $\mathrm{CFs}$.

\subsubsection{The Units?}

The unit of CFs for midpoint impact categories is specific for each category and LCIA method chosen, and therefore discussed in detail in the corresponding section dedicated in detail to each LCIA method in Chap. 40. However, two different approaches can be identified-expression in absolute form as the modelled indicator result (e.g. area of ecosystem exposed above its carrying capacity per $\mathrm{kg}$ of substance emitted for acidification) or expression in a relative form as that emission 
of a reference substance for the impact category which would lead to the same level of impact (e.g. $\mathrm{kg} \mathrm{CO}_{2}$-equivalents $/ \mathrm{kg}$ of substance emitted for climate change).

In contrast, endpoint CFs are typically expressed in absolute units and the units are relatively common between those LCIA methods that cover endpoint modelling:

Human health: [years] expressed as DALY (Disability-Adjusted Life Years). This unit is based on a concept proposed by Murray and Lopez (1996) and used by the World Health Organisation. It considers different severity contributions defined as "Years of Life Lost per affected Person" YLL $\mathrm{p}_{\mathrm{p}}$ [year/disease case] and "Years of Life lived with a Disability per affected Person" $\mathrm{YLD}_{\mathrm{p}}$ [years/disease case]. These statistical values are calculated on the basis of number and age of deaths (YLL) and disabilities (YLD) for a given disease. This information can be/combined into a single indicator using disability weights for each type of disability to yield the "Disability Adjusted Life Years per affected Person" DALY ${ }_{\mathrm{p}}$ [year/person].

Natural environment or ecosystems: $\left[\mathrm{m}^{2}\right.$ year] or $\left[\mathrm{m}^{3}\right.$ year] expressed as Potentially Disappeared Fraction (PDF). It can be interpreted as the time and area (or volume) integrated increase in the disappeared fraction of species in an ecosystem [dimensionless] per unit of midpoint impact indicator increase. It essentially quantifies the fraction of all species present in an ecosystem that potentially disappears (regardless whether due to death, reduced reproduction or immigration) over a certain area or volume and during a certain length of time. Different ecosystems have different numbers of species that can be affected by the impact and it is necessary to correct for such differences when aggregating the potentially disappeared fractions of species across the different impact categories at endpoint.

Resource depletion and ecosystem services: Different approaches exist and since there is still no common perception of what the area of protection for resources is (Hauschild et al. 2013), there is also no consensus forming on how to model damage in the form of resource depletion. Some proposals focus on the future costs for extraction of the resource as a consequence of current depletion, and these divide into costs in the form of energy or exergy use for future extraction (measured in MJ) or monetary costs (measured in current currency like USD, Yen or Euro).

\subsubsection{Uncertainties?}

Uncertainties can be important in LCIA and contribute substantially to overall uncertainty of an LCA result. For some impact categories, this contribution may be much larger than that of the LCI. At the same time, it is also crucial to be aware that large uncertainty is by no means a valid reason to exclude an impact category from the assessment. One of the more uncertain impact categories is human toxicity and it has to be capable of dealing with hundreds to thousands of different elementary flows, which may differ by more than 20 orders of magnitude in their impact potential, due to the sheer number of substances that may be assigned to this category and the variation in their environmental persistence and potential toxicity. 
It is much more certain to consistently characterise an impact category to which only a handful of elementary flows are assigned showing impact potentials that range only three or four orders of magnitude from the least to the most impacting elementary flow (e.g. eutrophication, acidification or global warming).

With the exception of photochemical ozone formation, there is no other impact category that covers even 100 different elementary flows. In this respect, there is hence a factor of $>1000$ between other impact categories and the toxicity categories (human health and ecotoxicity). This means that due to the large variety of substances with a toxicity potential, there will always be a very large uncertainty inherent in these categories, although developers will certainly be able to lower some of the model and parameter uncertainties currently observed. Excluding them from the assessment because of their uncertainty would therefore mean that toxicity would never be considered in LCA, which clearly risks violating the goal of LCA to avoid problem-shifting from one impact category to another. Besides, the uncertainty of assigning a zero-impact to a potentially toxic/elementary flow by neglecting the toxicity impact categories is certainly higher than the inherent uncertainty of the related characterisation factors.

The solution rather lies in the way we interpret such inherently uncertain impact potentials, whereas a more certain impact indicator may allow for identifying the exact contribution of each elementary flow to the total impact in this category, toxicity indicators allow for identifying the (usually 5-20) largest contributing elementary flows, which will constitute $>95 \%$ of the total impact. A further distinction between these will not be possible due to their uncertainty. Assuming that an average and complete LCI may contain several hundreds of potentially toxic elementary flows, one can then disregard all the remaining (several hundred) flows due to their low contribution to total toxicity. A further discussion and recommendations can be found in Rosenbaum et al. (2008).

Overall uncertainty in LCA is comprised of many different types of uncertainty as further discussed in Chap. 11. Variability (e.g. spatial or temporal/seasonal) may also be an important contributor, which should by principle be considered separately, as its contribution can be reduced to a large extent by accounting for it in the characterisation as discussed above for spatial variability and regionalised LCI and LCIA. Uncertainty in LCIA can only be reduced by improved data or model quality, essentially coming from updated LCIA methods, which is a good reason for a practitioner to keep up with the latest developments in LCIA, which may well lead to less uncertain results than the method one has been using for ten years. Most existing LCIA methods do not present information about the uncertainty of the characterisation factors.

\subsubsection{What Are the Main Assumptions?}

In current LCIA methods, some assumptions are considered as a basic requirement in the context of LCA: 
- Steady-state: Although exceptions exist, LCIA models are usually not dynamic (i.e. representing the variation of an environmental system's state over time and for specific time steps), but represent the environment as a system in steady state, i.e. all parameters which define its behaviour are not changing over time.

- Linearity: As life cycle inventory (LCI) data are typically not spatially and/or temporally differentiated, integration of the impact over time and space is required. In LCIA, this leads to the use of characterisation models assuming steady-state conditions, which implies a linear relationship between the increase in an elementary flow and the consequent increase in its potential environmental impact. In other words, e.g. doubling the amount of an elementary flow doubles its potential impact.

- Marginal versus average modelling: These terms are used in different ways and meanings in the LCA context; here they describe two different impact modelling principles or choices: a marginal impact modelling approach represents the additional impact per additional unit emission/resource extraction caused by the product system on top of the existing background impact (which is not caused by the modelled product system). This allows, e.g. considering nonlinearity of impacts depending on local conditions like high or low background concentrations to which the product systems adds an additional emission). An average impact modelling approach is strictly linear and represents an average impact independent from existing background impacts, which is similar to dividing the overall impact by the overall emissions. This is further discussed by Huijbregts et al. (2011). Note that marginal and averáge modelling are both suitable for small-scale interventions such as those related to a product or service. However, when medium-scale or large-scale interventions (or consequences) are to be assessed, the characterisation factors should represent non-marginal potential impacts and may also have to consider nonlinearity.

- Potential impacts: LCIA results are not actual or predicted impacts, nor exceedance of thresholds or safety margins, or risk. They are relative expressions of impacts associated with the life cycle of a reference unit of function (=functional unit), based on inventory data which are integrated over space and time, representing different locations and time horizons and based on impact assessment data which lack information about the specific conditions of the exposed environment.

- Conservation of mass/energy and mass/energy balance: Mass/energy cannot be created or disappear, it can only be transferred. Following this principle, processes of transport or transformation of mass or energy are modelled assuming that the mass/energy balance is conserved at all times.

- Parsimony: This refers to the basic modelling principle of "as simple as possible and as complex as necessary", an ideal balance that applies to LCIA characterisation models as well as to the entire LCA approach.

- Relativity: LCA results are relative expressions of impacts that relate to a functional unit and can be compared between different alternatives providing the same function (e.g. option A is more environmentally friendly than option B). 
An absolute interpretation of LCA results (e.g. option A is sustainable, option B is not) is not advisable as it requires a lot of additional assumptions.

- Best estimates: A fundamental value choice in LCA is not to be conservative, precautionary or protective, but to focus on avoiding any bias between compared scenarios by assuming average conditions, also referred to as best estimates. Products or services assessed in LCA are typically not representing one specific example (e.g. with a serial number or from a specific date), but an average, often disregarding whether a specific life cycle process took place in summer or winter, during the day or night, etc. As discussed by Pennington et al. (2004), LCA is a comparative assessment methodology. Direct adoption of conservative regulatory methodology and data is often not appropriate, and should be avoided in LCIA in order not to bias comparison between impact categories where different levels of precaution may be applied.

\subsection{Optional Steps According to ISO 14040/14044}

\subsubsection{Normalisation}

The indicator scores for the different midpoint impact categories are expressed in units that vary between the categories and this makes it unfeasible to relate them to each other and to decide which of them are large and which small. To support such comparisons, it is necessary to put them into perspective, and this is the purpose of the normalisation step, where the product system's potential impacts are compared to those of a reference system like a country, the world or an industrial sector. By relating the different impact potentials to a common scale they can be expressed in common units, which provide an impression of which of the environmental impact potentials are large and which are small, relative to the reference system. Normalisation can be useful for:

- Providing an impression of the relative magnitudes of the environmental impact potentials

- Presenting the results in a form suitable for a subsequent weighting

- Controlling consistency and reliability

- Communicating results

Typical references are total impacts per impact category per:

- Geographical zone which can be global, continental, national, regional or local

- Inhabitant of a geographical zone (e.g. expressing the "environmental space" occupied per average person)

- Industrial sector of a geographical zone (e.g. expressing the "environmental space" occupied by this product system relative to similar industrial activities) 
- Baseline reference scenario, such as another product system (e.g. expressing the "environmental space" occupied by this product system relative to a similar reference system using best available technology)

Using one of the first three reference systems listed above is also referred to as external normalisation. Using the last reference system in the list is also called internal normalisation when the reference scenario is one of the compared alternatives, such as the best or worse of all compared options or the baseline scenario representing, e.g. a current situation that is intended to be improved or a virtual or ideal scenario representing a goal to be reached. Normalised impact scores when using internal normalisation are often communicated as percentages relative to the reference system. In the illustrative case on window frames in Chap. 39 an internal normalisation is applied using the wooden frame window as reference (indexing it to $100 \%$ ) to reveal how the studied alternatives compare to this baseline choice. The study also applies external normalisation in order to compare the size of the different midpoint impact scores with the European person equivalent impact scores that is provided as default normalisation references for the LCIA method applied in the study (the ILCD method).

In practice, an LCIA method generally provides normalisation factors for use with its characterisation factors. The normalisation factors should be calculated using the same characterisation factors for the reference inventory as used for the inventory of the product system. Normalisation factors from different LCIA methods thus cannot be mixed or combined with characterisation factors from another LCIA method. This means that as an LCA practitioner you are usually limited to the reference system chosen by the LCIA method developers. Normalisation is applied using normalisation factors (NF). These are essentially calculated per midpoint and/or endpoint impact category by conducting an LCI and LCIA on the reference system, i.e. quantifying all environmental interventions $\mathrm{E}$ for all elementary flows $\mathrm{i}$ for the reference system and applying the characterisation factors CF per elementary flow i, respectively, for each impact category $c$. Although not obligatory, the normalisation reference is typically divided by the population $P$ of the reference region $r$, in order to express the NF per average inhabitant of the reference region (per capita impacts or "person equivalents"). This way, a total impact of the reference system per impact category is calculated, resulting in one NF per impact category $c$ :

$$
\mathrm{NF}_{c}=\left(\frac{\sum_{i}\left(\mathrm{CF}_{i} \cdot E_{i}\right)}{P_{r}}\right)^{-1}
$$

Ensuring consistency, the LCI data used to calculate a NF need to represent a common reference year and duration of activity (typically one year, being the reference year) for all impact categories. This results in NF having a unit expressing an impact per person and year, also referred to as person equivalent. A normalised impact score NS for a product system is calculated by multiplying the calculated impact score IS for the product system by the relevant NF per impact category $c$ : 


$$
\mathrm{NS}_{c}=\mathrm{IS}_{c} \cdot \mathrm{NF}_{c}
$$

Two different approaches exist for collection of inventory data for the calculation of NFs (with the exception for global NFs, where both approaches give equal results):

- Production-based (or top-down), representing the interventions taking place in the reference region as result of the total activities in the region

- Consumption based (or bottom-up), representing the interventions that are caused somewhere in the world as consequence of the consumption taking place in the reference region (and thus representing the demand for industrial and other activities within and outside the reference region)

Other ways to derive NF (although somewhat bordering to weighting already) are to base them on a conceptual "available environmental space". This can be determined using, e.g. political targets for limits of environmental interventions or impacts for a given duration and reference year (i.e. "politically determined environmental space" being the average environmental impact per inhabitant if the political reduction targets are to be met), or a region's or the planet's carrying capacity (i.e. "environmental space" being the amount of environmental interventions or impacts that the region or planet can buffer without suffering changes to its environmental equilibrium within each impact category). The latter would require knowing the amount of impact that a region or the planet can take before suffering permanent damage, which is a concept associated with much ambiguity and hence very uncertain to quantify. There is increasing focus on science-based targets in the environmental regulation with the $2^{\circ}$ ceiling for climate change as the most prominent example, and this may lead to future consensus building on science-based targets also for some of the other impacts that are modelled in LCIA. Political targets are often determined at different times and apply to different periods of time. In order to ensure a consistent treatment of each impact category, it is necessary to harmonise the target values available so that all targets for any given intervention are converted to apply to the same period and reference year. The targets can be harmonised by interpolating or extrapolating to a reduction target for a common target year, computed relative to interventions in the reference year. More details can be found in Hauschild and Wenzel (1998).

Caution is required when interpreting normalised LCA results! Applying normalisation harmonises the metrics for the different impact potentials and brings them on a common scale, but it also changes the results of the LCA and consequently may change the conclusions drawn from these. Since there is no one objectively correct choice of reference systems for normalisation, the interpretation of normalised LCA results must therefore always be done with due consideration of this choice of normalisation reference. A few main issues that need to be considered when interpreting normalised LCA results are:

- Depending on the size of and activities reflected in the reference system, different biases may be introduced in the comparison of the impact scores of a 
product system. As a general principle, the larger the reference system, the less the risk of such bias when normalising against the background activities of society

- While supporting comparison of results across impact categories, normalised LCA results cannot be interpreted as reflecting a weight or importance of one impact category relative to others. Normalisation helps to identify the impacts from the product system that are large compared to the chosen reference system, but the large is not necessary the same as important. It is therefore not suitable as the only basis for identification of key issues/impacts in a product system, unless explicitly required by the goal and scope definition (e.g. evaluating the environmental impact contribution of a product system to a reference system which it is part of)

- Unless (a) the reference system is global or (b) all environmental interventions of the product system assessed take place in the same region as those of the reference system, the direct interpretation of normalised impacts as contributions to or fractions of the reference system is misleading because parts of the life cycle of the product or service take place in different regions of the world, including outside the reference system

By expressing the different impact scores on a common scale, normalisation can also help checking for potential errors in the modelling of the product system. If the results are expressed in person equivalents, it is possible to spot modelling errors leading to extremely high or low impacts in some of the impact categories-like frequent unit errors when emissions are expressed in $\mathrm{kg}$ instead of $\mathrm{g}$. Looking across the impact category results in a normalised impact profile, it is also possible for the more experienced LCA practitioner to check whether they follow the pattern that would be expected for this type of product or service.

Although characterisation at endpoint level leads to much fewer impact scores (typically three), normalisation may still be useful with the same purposes as normalisation at midpoint level. The calculation and application of the endpoint normalisation references follows the same procedure as for midpoint normalisation, just applying combined midpoint and endpoint characterisation factors in Eq. 10.2.

\subsubsection{Weighting (and Aggregation)}

Weighting can be used to determine which impacts are most important and how important they are. This step can only be applied after the normalisation step and allows the prioritisation of impact categories by applying different or equal weights to each category indicator. It is important to note that there is no scientific or objective basis for this step. This means that, no matter which weighting method or scheme is applied, it will always be based on the subjective choices of one person or a group of individuals. Weighting can be useful for: 
- Aggregating impact scores into several or one single indicator (note that according to ISO 14040/14044 there is no scientific basis on which to reduce the results of an LCA to a single result or score because of the underlying ethical value-choices)

- Comparing across impact categories

- Communicating results applying an underlying prioritisation of ethical values

Note that in all of these cases weighting is applied, either implicitly or explicitly! Even when applying no explicit weighting factors in the aggregation, there is always an implicit equal weighting (all weighting factors $=1$ ) inherently applied when doing any of the above. According to ISO 14044, weighting is not permitted in a comparative assertion disclosed to the public and weighted results should always be reported together with the non-weighted ones in order to maintain transparency. The weighting scheme used in an LCA needs to be in accordance with the goal and scope definition. This implies that the target group including their preferences and the decisions intended to be supported by the study need to be considered, making shared values crucial for the acceptance of the results of the LCA. This can pose important problems due to the variety of possible values among stakeholders, including:

- Shareholders

- Customers

- Employees

- Retailers

- Authorities

- Neighbours

- Insurance companies

- NGOs (opinion leaders)

- ...

It may not be possible to arrive at weighting factors that will reflect the values of all stakeholders so focus will typically have to be on the most important stakeholders, but is it possible to develop one set of weighting factors that they will all agree on? If this is not the case, several sets of weighting factors may have to be applied, representing the preferences of the most important stakeholder groups. Sometimes the use of the different sets will lead to the same final recommendations which may then satisfy all the main stakeholders. When this is not the case, a further prioritisation of the stakeholders is needed, or the analysed product system (s) must be altered in a way that allows an unambiguous recommendation across the applied weighting sets.

The weighting of midpoint indicators should not be purely value-based. More, to some extent, science-based criteria for importance of environmental impacts may be:

- Probability of the modelled consequences, how certain are we on the modelled cause-effect relations?

- What is the resilience of the affected systems? 
- Existence of impact thresholds - in the characterisation modelling we typically assume linear cause-effect relationships for the small interventions in the product system but in the full environmental scale, there may be impact levels that represent tipping points beyond which much more problematic effects occur

- If so, then how far are we from such critical impact levels - is this an important concern in the near future?

- Severity of effect and gravity of consequences-disability, death, local extinction, global extinction

- Geographical scale

- Population density is essential for the impacts on human health

- Possibility to compensate/adapt to impact

- Temporal aspects of consequences - when will we feel the consequences, and for how long?

- Is the mechanism reversible, can we return to current conditions if we stop the impacts?

Indeed, many of these science-based criteria are attempted to be included in the environmental modelling linking midpoint indicators to endpoint indicators, and midpoint-to-endpoint characterisation factors may thus be seen as science-based weighting factors for the midpoint impact categories.

Different principles applied to derive weighting factors are:

- Social assessment of the damages (expressed in financial terms like willingness to pay), e.g. Impact on human health based on the cost that society is prepared to pay for healthcare (e.g. used in EPS and LIME LCIA methods)

- Prevention costs (to prevent or remedy the impact through technical means), e.g. the higher the costs, the higher the weighting of the impact

- Energy consumption (to prevent or remedy the impact through technical means), e.g. the higher the energy consumption, the higher the weighting of the impact

- Expert panel or Stakeholder assessment, e.g. weight attributed based on the relative significance, from a scientific perspective (subjective to each expert), of the different impact categories

- Distance-to-target (politically or scientifically defined): degree at which the targeted impact level is reached (distance from the target value), the greater the distance, the more weight is assigned to the impact (e.g. used in EDIP, Ecopoints and Swiss Ecoscarcity LCIA methods).

- Social science-based perspectives, not representing the choices of a specific individual, but regrouping typical combinations of ethical values and preferences present in society into a few internally consistent profiles (e.g. used in ReCiPe and Ecoindicator99 LCIA methods).

The latter approach is relatively widely used and applies three cultural perspectives, the Hierarchist, the Individualist and the Egalitarian (a forth perspective, the Fatalist is not developed for use in LCA since the fatalist is expected not to be represented among decision-makers, targeted by an LCA. For each cultural perspective coherent choices are described in Table 10.2 for some of the central 
Table 10.2 Cultural perspectives represented by preference with coherent choices (Hofstetter 1998)

\begin{tabular}{l|l|l|l}
\hline & Time perspective & Manageability & $\begin{array}{l}\text { Required level of } \\
\text { evidence }\end{array}$ \\
\hline H (Hierarchist) & $\begin{array}{l}\text { Balance between short } \\
\text { and long term }\end{array}$ & $\begin{array}{l}\text { Proper policy can avoid } \\
\text { many problems }\end{array}$ & $\begin{array}{l}\text { Inclusion based on } \\
\text { consensus }\end{array}$ \\
\hline I (Individualist) & Short time & $\begin{array}{l}\text { Technology can avoid } \\
\text { many problems }\end{array}$ & Only proven effects \\
\hline E (Egalitarian) & Very long term & $\begin{array}{l}\text { Problems can lead to } \\
\text { catastrophe }\end{array}$ & All possible effects \\
\hline
\end{tabular}

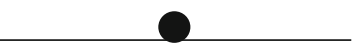

Nature capricious

(Fatalistís View)

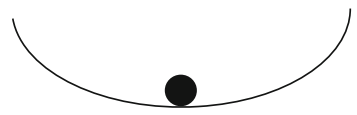

Nature Benign (Individualistís View)

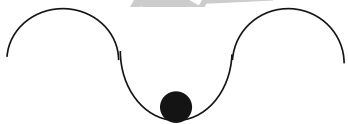

Nature Perverse/Tolerant (Hierarchistís View)

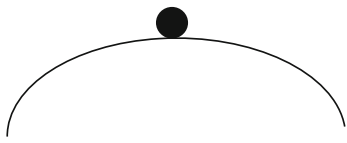

Nature Ephemeral (Egalitarianís View)

assumptions made in the characterisation modelling and in the development of a set of consistent weighting factors for each archetype.

The different archetypal views on nature and the related risk perceptions are illustrated in Fig. 10.3. The dot represents the state of nature as a rolling ball, shifted by human activities along the curve representing nature's reaction to a shift. Its position in the figures indicates the state of harmony between humans and nature according to the four archetypal views.

\subsubsection{Grouping}

This step consists in placing the impact categories in one or several groups or clusters (as defined in goal and scope) and can involve sorting or ranking, applying one of two possible methods: 
- Sorting and clustering midpoint impact categories on a nominal basis (e.g.: by characteristics such as emission-related and resource-related, or global, regional or local spatial scales)

- Ranking the impact categories according to a set (subjective-based on ethical value-choices) hierarchy (e.g.: high, medium or low priority)

\subsection{Footprints Versus LCA}

"I was exceedingly surprised with the print of a man's naked foot on the shore, which was very plain to be seen in the sand." (Daniel Defoe, Robinson Crusoe, 1719). The meaning of the term "footprint" has largely evolved since Daniel Defoe's famous novel and is currently used in several contexts (Safire 2008). Its appearance in the environmental field can be tracked back to 1992 when William Rees published the first academic article on the thus-termed "ecological footprint" (Rees 1992), which was further developed by him and Mathis Wackernagel in the following years. Its aim is to quantify the mark left by human activities on natural environment.

Since then, the mental images created by the word has contributed to its use as an effective way of communicating on different environmental issues and raising environmental awareness within the scientific community as well as among policy communities and the general public. Since the early 2000s, several footprints have thus emerged within the environmental field with different definitions and meanings, ranging from improved ecological footprint methodologies to the representation of specific impacts of human activities on ecosystems or human health to a measure of a specific resource use. Prominent examples are:

- Ecological footprint focusing on land use (http://www.footprintnetwork.org)

- Cumulative Energy Demand (CED) focusing on non-renewable energy

- Material Input Per unit of Service (MIPS) focusing material use

- Water footprint focusing on water use volumetric accounting (http:// waterfootprint.org)

- Water footprint focusing on water use impacts including pollution (ISO 14046)

- Carbon footprint focusing on climate change (ISO 14064, ISO/TS 14067, WRI/WBCSD GHG protocol, PAS 2050)

Later developments focused on the introduction of new environmental concerns or enlarging the scope of footprints. Examples for such emerging footprints are:

- Chemical footprint focusing on toxicity impacts

- Phosphorus depletion footprint

As illustrated in Fig. 10.4, all footprints are fundamentally based on the life cycle perspective and most of them focus on one environmental issue or area of concern. 

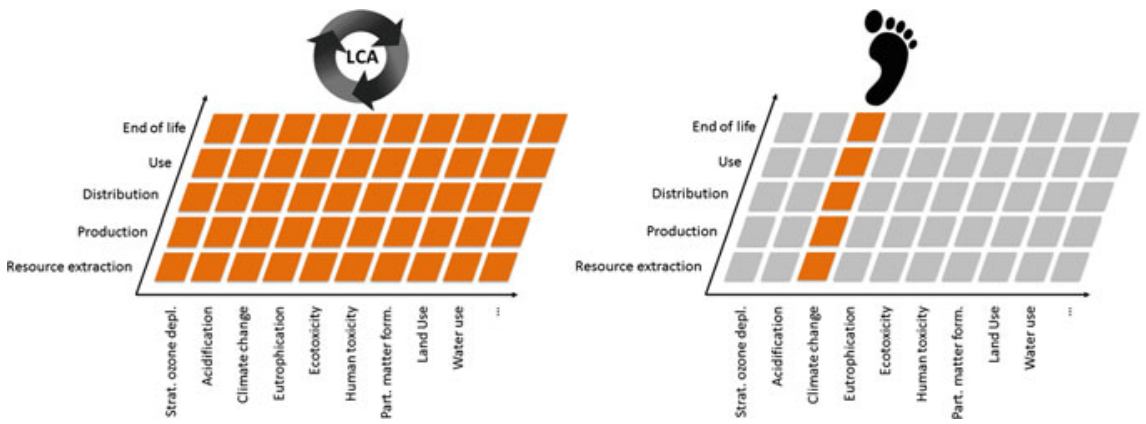

Fig. 10.4 The fundamental difference in scope and completeness between LCA and footprints while both apply the life cycle perspective

They can be applied to a large variety of assessment targets like products, services, organisations, persons and populations, sites and regions, even countries or the entire world. Their success in the last decades lies in their particular strengths:

- Easily accessible and intuitive concept

- Easy to communicate about specific environmental issues or achievements with non-environmental experts (policy and decision-making communities, general public)

- Availability of data

- Easy to perform

- Wide range of assessment targets can easily be assessed

These strengths, however, also come with a number of important limitations:

- Their focus on one environmental issue does not inform about a potential burden-shifting from one environmental issue (e.g. climate change) to another (e.g. water availability). Therefore, while they allow for identification of the best option for one environmental problem, they are not suitable to support decisions regarding environmental sustainability, which need to consider all potential environmental problems

- Some footprints only assess the quantity of a resource used (e.g. ecological footprint, CED, MIPS and volumetric water footprint), which is comparable to the accounting of quantities used or emitted in the life cycle inventory (see Chap. 9). Such footprints therefore do not inform about the associated environmental consequences of the resources used or emissions accounted, and they do not quantify potential impacts on a given area of protection. Among other, this limitation compromises the comparability of footprints for different options to choose from

- Impact-básed footprints (e.g. carbon footprint), at least historically, assess impacts on midpoint level and hence do not reflect damages, which has implications on their environmental relevance. However, with an increasing 
range of endpoint impact indicators available, this may be solved with science advancing further

- Different footprints can usually not be combined to enlarge their environmental scope because their system boundaries (see Chaps. 8 and 9) are not aligned and double counting of impacts becomes likely, which increases the risk of bias to the comparison, the same way the omission of impacts does

As mentioned above, the focus on single environmental problems has important implications regarding the risks of using footprints in decision-making processes. A study by Huijbregts et al. (2008) calculated 2630 product-specific ecological footprints of products and services (e.g. energy, materials, transport, waste treatment, etc.). They concluded that "Ecological footprints may [...] serve as a screening indicator for environmental performance... [and provide] a more complete picture of environmental pressure compared to non-renewable CED [Cumulative Energy Demand]", while also observing that "There are cases that may [...] not be assessed in an adequate way in terms of environmental impact. For example, a farmer switching from organic to intensive farming would benefit by a smaller footprint for using less land, while the environmental burdens from applying more chemicals [i.e. pesticides and fertilisers] would be neglected". Thus, the usefulness of the ecological footprint as a stand-alone indicator may often be limited (Huijbregts et al. 2008).

The limitations of carbon footprints (i.e. the climate change impact indicator in LCA) as environmental sustainability indicators was investigated by a study from Laurent et al. (2012), who assessed the carbon footprint and 13 other impact scores from 4000 different products, technologies and services (e.g. energy generation, transportation, material production, infrastructure, waste management). They found "that some environmental impacts, notably those related to emissions of toxic substances, often do not covary with climate change impacts. In such situations, carbon footprint is a poor representative of the environmental burden of products, and environmental management focused exclusively on [carbon footprint] runs the risk of inadvertently shifting the problem to other environmental impacts when products are optimised to become more "green". These findings call for the use of more broadly encompassing tools to assess and manage environmental sustainability" (Laurent et al. 2012).

This problem is demonstrated in Fig. 10.5, which shows the carbon footprint, ecological footprint, volumetric water footprint and the LCA results for an illustrative comparison of two products $\mathrm{A}$ and $\mathrm{B}$. If one had to choose between option $\mathrm{A}$ and $\mathrm{B}$, the decision would be different and thus depending on, which footprint was considered, whereas LCA results provide the full range of potential impacts to consider in the decision.

The large variety in footprints and their definitions and methodological basis in combination with their wide use in environmental communication and marketing claims, has resulted in confusing and often contradictory messages to buyers. This ultimately limited the development and functioning of a market for green products (Ridoutt et al. 2015, 2016). In response, a group of experts established under the 


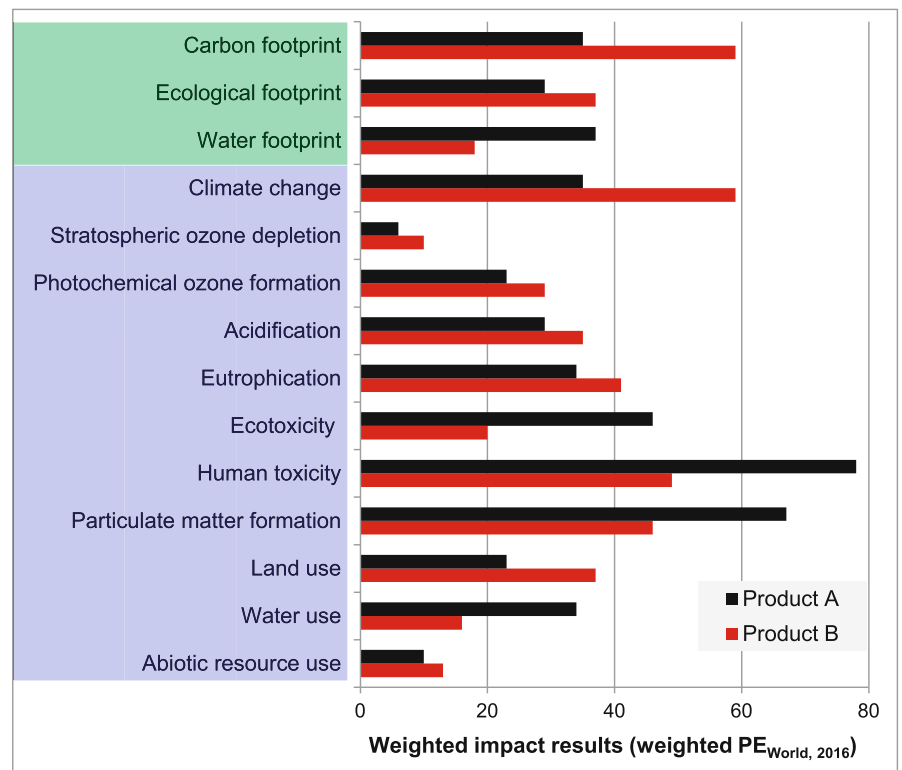

Fig. 10.5 Comparing two products, which alternative would you choose? Examples of footprints are indicated in green shading; impact categories commonly assessed in LCA are indicated in blue shading

auspices of the UNEP/SETAC Life Cycle Initiative defined footprint as "Metric used to report life cycle assessment results addressing an area of concern [the latter specified as an] Environmental topic defined by the interest of society" (Ridoutt et al. 2016). This definition underpins a footprint's focus on environmental issues particularly perceived by society (e.g. climate change or water scarcity) and allows for a clear distinction to LCA, which is primarily oriented "toward stakeholders interested in comprehensive evaluation of overall environmental performance and trade-offs among impact categories" (Ridoutt et al. 2016) and related areas of protection. This definition also recognises the inherent complexity of an environmental performance profile resulting from an LCA study, which requires a certain expertise to be correctly interpreted.

In conclusion, footprints are life cycle-based, narrow-scoped environmental metrics focusing on an area of concern. They are widely and easily applicable, as well as easily understood by non-environmental experts and therefore straightforward to communicate. They are particularly useful for communication of environmental problems or achieved improvements, as long as their use is restrained to their coverage of environmental concerns and care is taken when interpreting them (burden-shifting), particularly when results are disclosed to non-expert audiences (e.g. public opinion). A footprint's life cycle perspective can be an inspiring first contact with the concept of life cycle thinking for the general public, and for policy and decision-makers it often serves as an entry-door into the concept and 
methodology of LCA. Footprints have the ability to raise environmental awareness and therefore are springboards towards the use of more-encompassing assessment tools such as LCA. They can constitute a first step for organisations or companies, who can already implement procedures as a preparation for full environmental assessments. However, due to a footprint's narrow scope and limited representativeness for a comprehensive set of environmental indicators, they are not suitable for decision-support of any kind including product labels, ecodesign, policy-support and the like.

\subsection{Detailed Description of Impact Categories Currently Assessed in LCA}

The following sections document how the most commonly considered environmental problems (i.e. impact categories) are handled in life cycle impact assessment. Ionising radiation is also a commonly addressed impact category in LCA, but was not included in the detailed overview here due to its specificity to a limited number of processes in the LCI. The impact categories are dealt with in sequence going from global over regional towards local and addressing first the emission-related and then the extraction-related categories. The common structure of the sections are:

- What is the problem?

- What is the underlying environmental mechanism and how is it modelled in LCIA?

- What are the human activities and elementary flows contributing most to the problem? (emission-based categories only)

- What are the most widely used, existing LCIA characterisation models?

Beyond the classic list of impact categories discussed hereafter, there is a number of emerging categories currently in the stage of research and development. Though potentially relevant they have not yet reached sufficient methodological maturity to be operational for the majority of practitioners and no or only few LCIA methods have included them in their indicator set. Some examples are:

- Biotic resources such as fish or wood

- Noise

- Pathogens

- Salinization

- Accidents

- Impacts of Genetically Modified Organisms (GMO).

A profound comparison of existing LCIA methods was performed by Hauschild et al. (2013) for the establishment of recommended LCIA models for the European context. Taking Hauschild et al.'s work as a starting point, the tables in Chap. 40 
provide a complete and updated qualitative comparison of widely used LCIA methods available in current LCA software.

\subsection{Climate Change}

\subsubsection{Problem}

The greenhouse effect of our atmosphere, discovered and explored from the early $19^{\text {th }}$ century, is vital to life on our planet and has always existed since the dawn of life on Earth. Without it the global average temperature of our atmosphere near the ground would be $-18{ }^{\circ} \mathrm{C}$ instead of currently $15{ }^{\circ} \mathrm{C}$. Hence, there are natural drivers and sources keeping it in balance (with periodical imbalances leading to natural events such as ice ages). In addition to those, anthropogenic activities also contribute to this effect increasing its intensity and creating global warming, which refers to the phenomenon of rising surface temperature across the planet averaged over longer periods of time. The Intergovernmental Panel on Climate Change (2014a) (IPCC) defines climate change as "a change in the state of the climate that can be identified (e.g. using statistical tests) by changes in the mean and/or the variability of its properties, and that persists for an extended period, typically decades or longer". IPCC observed an acceleration of the rise in planetary surface temperature in the last five to six decades, with the highest rates at the very northern latitudes of the Arctic. Ocean temperatures are also on the rise down to a depth of at least $3000 \mathrm{~m}$ and have so far absorbed most of the heat trapped in the atmosphere. Tropospheric temperatures are following similar trends as the surface. Although, still debated by few sceptics, most scientists agree on the presence of this effect with anthropogenic activities as the main cause. These are also the focal point of LCIA methodology and hence of this chapter.

Effects observed by IPCC with varying degrees of confidence based on statistical measures (IPCC 2014a):

- Rise of atmospheric temperature with the last three decades from 1983 to 2012 being very likely the warmest 30 -year period of the last 800 years in the Northern Hemisphere and likely the warmest 30-year period of the last 1400 years

- Rise of ocean temperature in the upper $75 \mathrm{~m}$ by a global average of $0.11^{\circ} \mathrm{C}$ per decade from 1971 to 2010

- Melting of glaciers, snow and ice caps, polar sea ice and ice packs and sheets ( $\neq$ polar sea ice) and permafrost soils

- Rise in global mean sea levels by $0.19 \mathrm{~m}$ over the period 1901-2010 (due to thermal expansion and additional water from melting ice)

- Increase in frequency and intensity of weather-based natural disasters, essentially due to increased atmospheric humidity and consequent changes in 
atmospheric thermodynamics (i.e. energy absorption via evaporation and condensation) and cloud formation

- Intense tropical cyclone activity increased in the North Atlantic since 1970

- Heavy precipitation and consequent flooding (North America and Europe)

- Droughts

- Wildfires

- Heat waves (Europe, Asia and Australia)

- Alteration of hydrological systems affecting quantity and quality of water resources

- Negative impacts of climate change on agricultural crop yields more common than positive impacts

- Shifting of geographic ranges, seasonal activities, migration patterns, abundances and species interactions (including in biodiversity) by many terrestrial, freshwater and marine species

- Changes in infectious disease vectors

The continuation and intensification of already observed effects as well as those not yet observed (but predicted by models as potential consequences of further global warming) depend on the future increase in surface temperature which is predicted using atmospheric climate models and a variety of forecasted emission scenarios ranging from conservative to optimistic. Given the inertia of atmospheric and oceanic processes and the global climate, it is expected that global warming will continue over the next century. Even if emissions of GHGs would stop immediately, global warming would continue and only slow down over many decades. The following effects are not yet observed and highly debated in the scientific community; hence consensus or general agreement regarding their likelihood is not established. Nevertheless, they are possible impacts and should be seen as part of the possible effects of global warming, especially when considering longer time horizons.

- Slowing down of the thermohaline circulation of cold and salt water to the ocean floor at high latitudes of the northern hemisphere (e.g. Gulf stream), among other things responsible for global heat distribution, oceanic nutrient transport, the renewal of deep ocean water, and the relative mildness of the European climate. This circulation as shown in Fig. 10.6 is driven by differences in the density of water due to varying salinity and differences in water temperature, and might be affected by freshwater inflow from melting ice, decreasing sea water salinity and consequently reducing its density and the density gradient between different oceanic zones.

- Increasing frequency and intensity of "El Niño" events while decreasing that of its counterpart "La Niña" might be possible, although it is unclear to what extent this is influenced by global warming. One possibility is that this effect only occurs in the initial phase of global warming, while weakening again later when the deeper layers of the ocean get warmer as well. Dramatic changes cannot be fully excluded based on current evidence; therefore, this effect is considered a potential tipping element in our climate. 


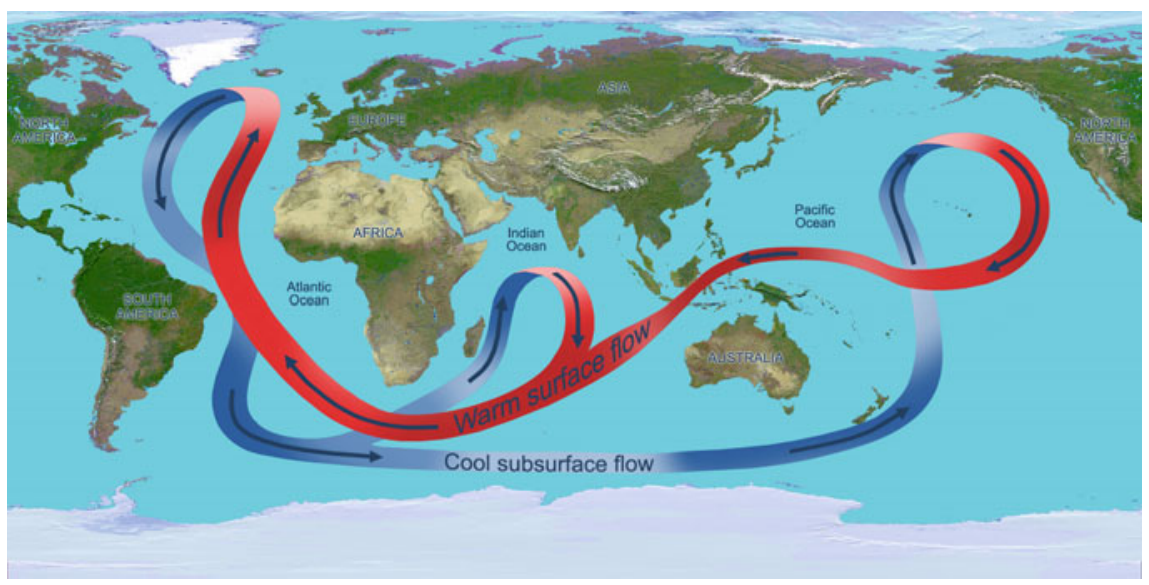

Fig. 10.6 "The big loop" takes 1500 years to circumnavigate the globe (NASA/JPL 2010, public domain, http://www.jpl.nasa.gov/news/news.php?release=2010-101)

- Mobilisation and release of oceanic methane hydrate (water ice containing large amounts of methane in its crystal structure) present in deep ocean sediments and permafrost, could lead to further global warming and significantly affect the atmospheric oxygen content. There is large uncertainty regarding the amounts and size of reserves found under sediments on the ocean floors, but a relatively sudden release of large amounts of methane hydrate deposits is believed to be a main factor in the global warming of $6{ }^{\circ} \mathrm{C}$ during the end-Permian extinction event (Benton and Twitchet 2003) when $96 \%$ of all marine species became extinct 251 million years ago.

- Effects on Earth's primary "lung": phytoplankton which produces $80 \%$ of terrestrial oxygen and absorbs a significant share of $\mathrm{CO}_{2}$.

- In addition to the environmental effects discussed above, the human population is likely to be affected by further severe consequences should other adaptation strategies prove inefficient: disease, malnutrition and starvation, dehydration, environmental refugees, wars and ultimately death.

- Nonlinearity of cause-effect chains, feedback and irreversible tipping points: Although, in LCIA models, linearity of cause-effect chains is assumed, the above discussed effects present several examples of mechanisms that are unlikely to depend linearly on the temperature increase, i.e. they will not change proportionally in frequency and/or intensity per degree of change in global temperature. Furthermore, they are likely to directly or indirectly influence each other, causing feedback reactions adding further nonlinearity. Additionally, some of these effects will be irreversible, changing the climate from one stable state to another. This phenomenon is referred to as tipping points, and the above-mentioned release of methane from methane hydrates and the alteration of the Gulf stream are examples. Lenton et al. (2008) discuss a number of additional potential tipping points. 
- Forest dieback (Boreal forest, Amazon rainforest).

- Area encompassed by monsoon systems will increase with intensified precipitation.

\subsubsection{Environmental Mechanism}

In principle, the energy reaching the Earth's atmosphere from solar radiation and leaving it again (e.g. via reflection and infrared radiation) is in balance, creating a stable temperature regime in our atmosphere. As shown in Fig. 10.7, from the sunlight reaching the Earth's atmosphere, one fraction $(\sim 28 \%)$ is directly reflected back into space by air molecules, clouds and the surface of the earth (particularly oceans and icy regions such as the Arctic and Antarctic): this effect is called albedo. The remainder is absorbed in the atmosphere by greenhouse gases (GHG) $(21 \%)$ and the Earth's surface $(50 \%)$. The latter heats up the planetary surface and is released back into the atmosphere as infrared radiation (black body radiation) with a longer wave length than the absorbed radiation. This infrared radiation is partially absorbed by GHGs and therefore kept in the atmosphere instead of being released into space, explaining why the temperature of the atmosphere increases with its contents of GHGs.

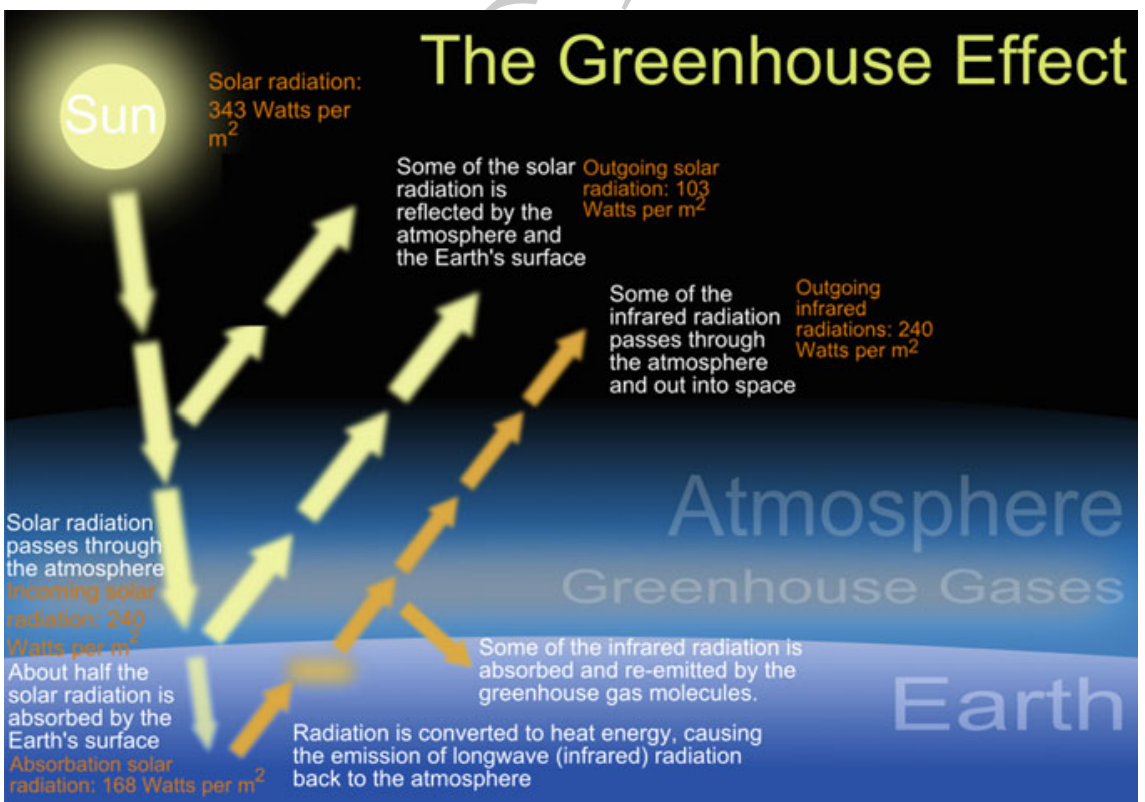

Fig. 10.7 The greenhouse effect (CUser: ZooFari/Wikimedia Commons/CC-BY-SA-3.0) 
A cause-effect chain for climate change is shown in Fig. 10.8 and can be summarised as follows:

1. GHG emissions

2. Transport, transformation and distribution of GHG in the atmosphere

3. Disturbance of the radiation balance-radiative forcing (primary effect, midpoint)

4. Increase in global temperatures of atmosphere and surface

5. Increase in sea level due to heat expansion and the melting of land-based ice

6. Increased water vapour content of the atmosphere causing more extreme weather

7. Negative effects on the ecosystems and human health (endpoint)

Until now the unanimously used climate change indicator on midpoint level in LCA has been the Global Warming Potential, an emission metric first introduced in the IPCC First Assessment Report (IPCC 1990) and continuously updated by IPCC

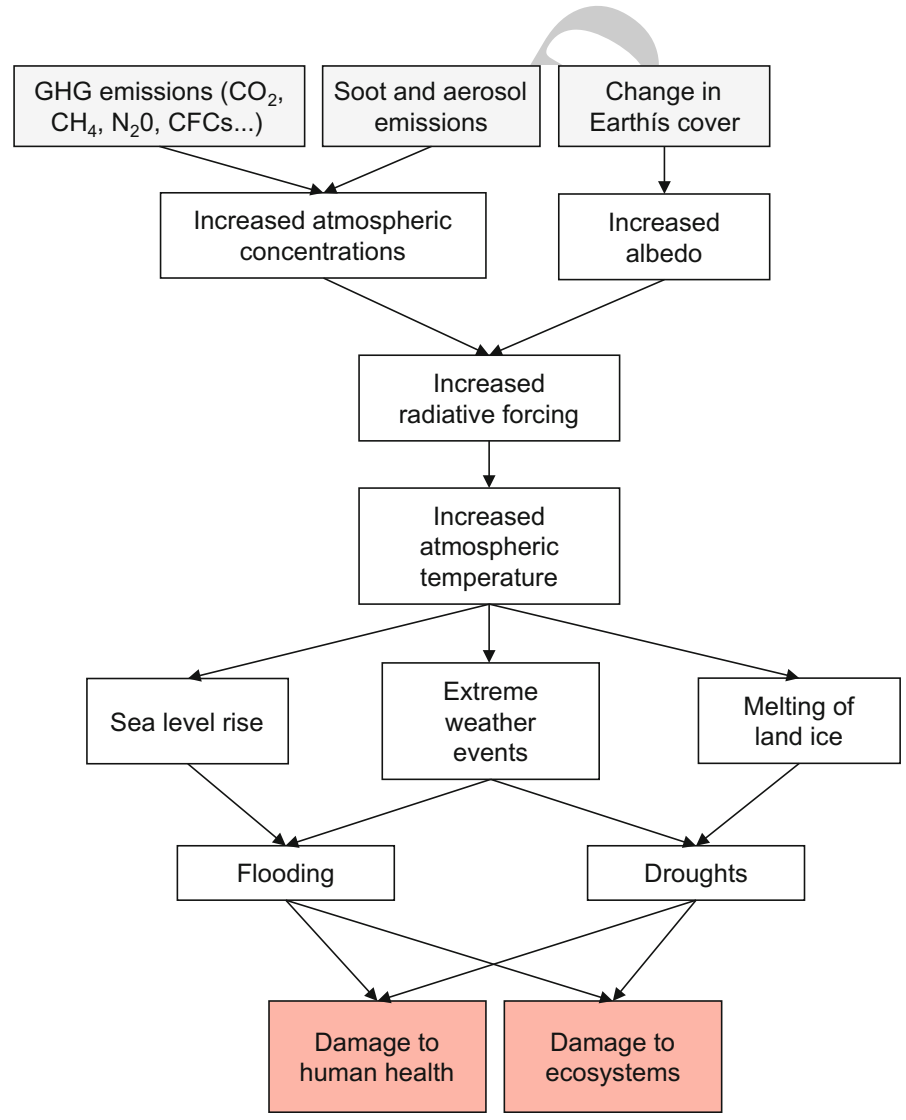

Fig. 10.8 Impact pathway for climate change 
since then with the latest version in the Fifth Assessment Report (IPCC 2013). Global warming potentials are calculated for each GHG according to:

$$
\mathrm{GWP}_{i}=\frac{\int_{0}^{T} a_{i} \cdot C_{i}(t) \mathrm{d} t}{\int_{0}^{T} a_{\mathrm{CO}_{2}} \cdot C_{\mathrm{CO}_{2}}(t) \mathrm{d} t}
$$

where

- $a_{i}$ : thermal radiation absorption (instant radiative forcing) following an increase of one unit in the concentration of gas $i$

- $C_{i}(t)$ : Concentration of gas $i$ remaining at time $t$ after emission

- $T$ : number of years for which the integration is carried out (e.g. 20 or 100 years)

GWP100-year is directly used in LCIA as the characterisation factor. As shown above, it is the ratio of the cumulated radiative forcing over 100 years of a given GHG and that of $\mathrm{CO}_{2}$, with the unit of $\mathrm{kg} \mathrm{CO}_{2}$-eq/kg GHG. Therefore, GWP for $\mathrm{CO}_{2}$ is always 1 and a GWP100 for methane of $28 \mathrm{~kg} \mathrm{CO}_{2}$-eq/kg methane (see Table 10.3) means that methane has 28 times the cumulated radiative forcing of $\mathrm{CO}_{2}$ when integrating over 100 years. The difference in GWP20 and GWP100 for methane shown in Table 10.3 is due to the fact that methane has a relatively short atmospheric lifetime of 12 years compared to $\mathrm{CO}_{2}$ 's lifetime which is at least one order of magnitude higher, which means that methane's GWP gets lower the longer the time horizon over which it is integrated (i.e. sort of a 'dilution' of its effect over a longer time). On the other hand a more persistent GHG such as nitrous oxide with 120 years lifetime has a similar value when integrating over 20 and 100 years and the 'time-dilution' effect would only become visible when integrating over time periods significantly longer than 120 years.

\subsubsection{Emissions and Main Sources}

Many greenhouse gases are naturally present in the atmosphere and contribute to the natural greenhouse effect. Estimated main contributors to the natural greenhouse effect are:

Table 10.3 Excerpt from the list of GWP (IPCC 2014a)

\begin{tabular}{|c|c|c|c|c|c|}
\hline \multirow[t]{2}{*}{ Substance } & \multirow[t]{2}{*}{ Molecule } & \multirow{2}{*}{$\begin{array}{l}\text { Atmospheric } \\
\text { lifetime } \\
\text { (years) }\end{array}$} & \multirow{2}{*}{$\begin{array}{l}\text { Radiative } \\
\text { efficiency } \\
\left(\mathrm{W} /\left(\mathrm{m}^{2} \mathrm{ppb}\right)\right)\end{array}$} & \multicolumn{2}{|c|}{$\begin{array}{l}\text { GWP }\left(\mathrm{kg} \mathrm{CO}_{2}-\mathrm{eq} / \mathrm{kg}\right. \\
\text { GHG) }\end{array}$} \\
\hline & & & & 20 years & 100 years \\
\hline Carbon dioxide & $\mathrm{CO}_{2}$ & & $1.37 \mathrm{E}-05$ & 1 & 1 \\
\hline Methane & $\mathrm{CH}_{4}$ & 12 & $3.63 \mathrm{E}-04$ & 84 & 28 \\
\hline Nitrous oxide & $\mathrm{N}_{2} \mathrm{O}$ & 121 & $3.00 \mathrm{E}-03$ & 264 & 265 \\
\hline
\end{tabular}


- Water vapour: $\sim 55 \%$

- Carbon dioxide $\left(\mathrm{CO}_{2}\right): 39 \%$

- Ozone $\left(\mathrm{O}_{3}\right): 2 \%$

- Methane $\left(\mathrm{CH}_{4}\right): 2 \%$

- Nitrous oxide $\left(\mathrm{N}_{2} \mathrm{O}\right): 2 \%$

Anthropogenic water vapour emissions do not contribute to climate change as the presence of water vapour is a function of atmospheric temperature and evaporation surfaces. For the other constituents however, anthropogenic sources for $\mathrm{CO}_{2}, \mathrm{CH}_{4}$ and $\mathrm{N}_{2} \mathrm{O}$ do contribute to increasing the greenhouse effect beyond its natural state. Further relevant GHG emissions also include industrial volatile and persistent halocarbons (chlorinated fluorocarbons including CFCs ("freons"), HCFCs and perfluoromethane) and sulphur hexafluoride ( $\left.\mathrm{SF}_{6}\right)$. GHG emissions are attributable to almost any human activity. The most important contributing activities are: burning of fossil fuels and deforestation (including releasing carbon from soil and change in albedo). Figure 10.9 shows the global contributions to GWP from five major economic sectors for the year 2010. Industry, agriculture, housing and transport are the dominating contributors to GHG emissions.

In addition to the greenhouse gases which all exert their radiative forcing in the atmosphere over timespans of years to centuries, there are also more short-lived radiative forcing agents that are important for the atmospheric temperature in a more short-term perspective. These include:

- Sulphate aerosols (particulate air pollution/caused by the emission of sulphur oxides from combustion processes) that reduce the incoming radiation from the sun and thus have a negative contribution to climate change

- Nitrogen oxides $\mathrm{NO}$ and $\mathrm{NO}_{2}$ (jointly called $\mathrm{NO}_{x}$ ) and $\mathrm{VOC}$ from combustion processes, that contribute to photochemical formation of ozone (see Sect. 10.10) which is a strong but short-lived radiative forcing gas

The radiative forcing impact of short-lived agents like these is very uncertain to model on a global scale, and their contribution to climate change is therefore not currently included in LCIA.

\subsubsection{Existing Characterisation Models}

All existing LCIA methods use the GWP (Eq. 10.4) for midpoint characterisation. In terms of time horizon most use 100 years, which has been recommended by IPCC as the best basis for comparison of GHGs, while some methods use a 500 year time horizon to better incorporate the full contribution from the GHGs. As mentioned, the longer time perspective puts a higher weight on long-lived GHGs like nitrous oxide, CFCs and $\mathrm{SF}_{6}$ and a lower weight on short-lived GHGs like methane. 


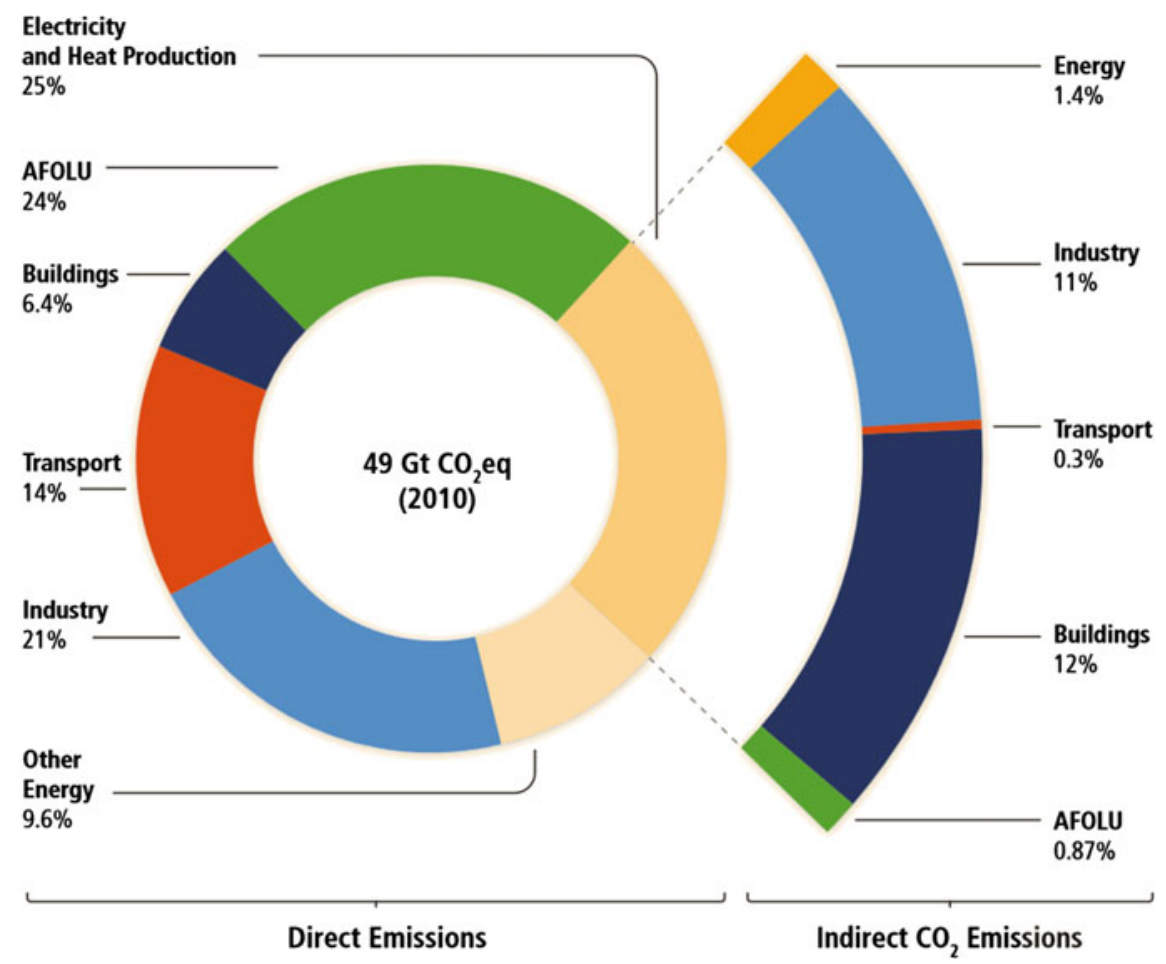

Fig. 10.9 Direct GHG emission shares (\% of total anthropogenic GHG emissions) of five major economic sectors in the world in 2010. The pull-out shows how indirect $\mathrm{CO}_{2}$ emission shares (in $\%$ of total anthropogenic GHG emissions) from electricity and heat production are attributed to sectors of final energy use. 'Other Energy' refers to all GHG emission sources in the energy sector other than electricity and heat production. 'AFOLU' stands for Agriculture, Forestry, and Other Land Use [taken from IPCC (2014b)]

So far radiative forcing agents with shorter atmospheric lifetime than methane are not considered in LCIA but a UNEP-SETAC expert workshop in 2016 recommended that climate change assessment at midpoint should be split into two sub-categories, respectively, focusing on the long-term climate change contributions and on the rate by which temperature changes occur. The two would be expressed in different metrics and not aggregated at midpoint level. It is expected that the distinction into two midpoint categories will cater better for the damage modelling since both rate of change and magnitude of the long-term temperature increase are important.

Endpoint characterisation of climate change is a challenge due to the complexity of the underlying environmental mechanisms with multiple feedback loops of which many/are probably unknown, the global scale and the very long time perspective. In particular damages to human health are also strongly affected by local and regional differences in vulnerability and ability of societies to adapt to changing 
climate conditions. Some endpoint methods have proposed endpoint characterisation factors (e.g. Ecoindicator99, ReCiPe, LIME, IMPACT World+ and LC-IMPACT), but due to the state of current climate damage models, they inevitably miss many damage pathways and are accompanied by very large uncertainties, where even the size of these uncertainties is difficult to assess. This is why other endpoint methods (e.g. IMPACT 2002+) refrain from endpoint modelling for this impact category and present the midpoint results for climate change together the endpoint results for the rest of the impact categories. In any case, endpoint results for climate change must be taken with the greatest caution in the interpretation of results. For further details see Chap. 40 and Hauschild and Huijbregts (2015).

\subsection{Stratospheric Ozone Depletion}

\subsubsection{Problem}

Ozone $\left(\mathrm{O}_{3}\right)$ is a highly reactive and unstable molecule consisting of three oxygen atoms and forms a bluish gas at normal ambient temperature with a distinct somewhat sharp odour. This molecule is present in lower atmospheric layers (tropospheric ozone as a consequence of photochemical ozone formation) and in larger concentrations (about $8 \mathrm{ppmv}$ ) also in higher altitudes between 15 and $40 \mathrm{~km}$ above ground (stratospheric ozone). Tropospheric, ground-level ozone is considered a pollutant due to its many harmful effects there on humans, animals, plants and materials (see Sect. 10.10). However, as a component of stratospheric atmospheric layers, it is vital to life on planet Earth, due to its capacity to absorb energy-rich UV radiation, thus preventing destructive amounts of it from reaching life on the planet's surface.

Stratospheric ozone depletion refers to the declining concentrations of stratospheric ozone observed since the late 1970s, which are observed in various ways: (1) As the 'ozone depletion area' or 'ozone hole' (an ambiguous term often used in public media referring to an area of critically low stratospheric ozone concentration), a recurring annual cycle of relatively extreme drops in $\mathrm{O}_{3}$ concentrations over the poles which start to manifest annually in the late winter/early spring of each hemisphere (i.e. from around September/October over the South pole and March/April over the North pole) before concentrations recover again with increasing stratospheric temperatures towards the summer. (2) A general decline of several percent per decade in $\mathrm{O}_{3}$ concentrations in the entire stratosphere. Ozone concentration is considered as critically low when the value of the integrated ozone column falls below 220 Dobson units (a normal value being about 300 Dobson units). Dobson Units express the whole of ozone in a column from the ground passing through the atmosphere. 'Ozone holes' have been observed over Antarctic since the early 1980s as shown in Fig. 10.10. 

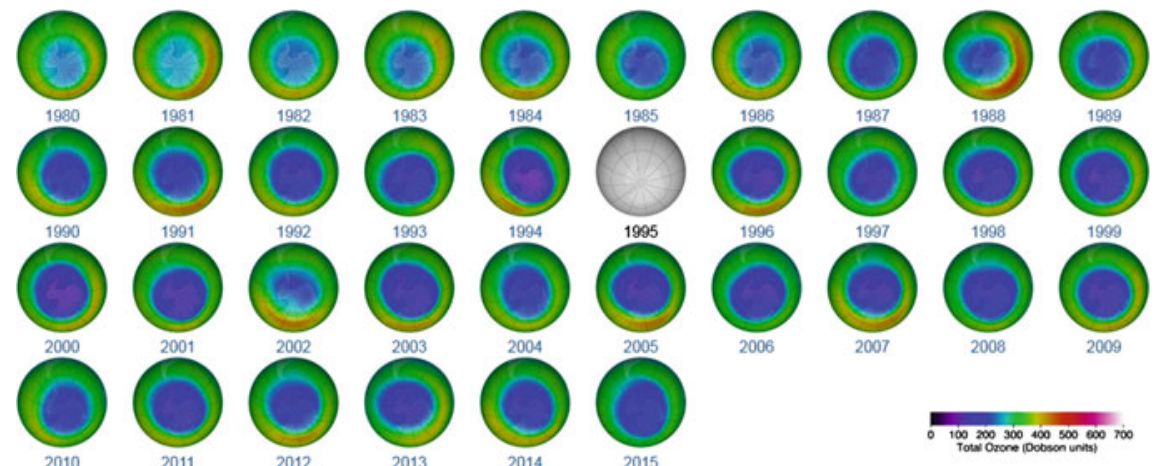

$100 \quad 200 \quad 300400 \quad 500 \quad 000700$

Fig. 10.10 Evolution of the hole in the ozone layer over Antarctica in September from 1980 to 2015 (Source NASA Ozone Watch 2016, public domain, http://ozonewatch.gsfc.nasa.gov/ monthly/climatology_09_SH.html)

Data for Europe for example show a decline of $5.4 \%$ of stratospheric $\mathrm{O}_{3}$ concentration per decade since the 1980s when measured in winter and spring, with an improving trend over the period 1995-2000. However, in later years low concentration records were broken on an almost annual basis. To date, the largest 'ozone hole' in human history was observed in 2006 with 29.5 million $\mathrm{km}^{2}$ over Antarctica, but even in 2015 its largest spread still reached 28.2 million $\mathrm{km}^{2}$. The largest Arctic 'ozone hole' ever was observed in 2011.

Impacts of stratospheric ozone depletion are essentially linked to reduced absorption of solar radiation in the stratosphere leading to increased UV radiation intensities at the planet surface, of which three broad (wavelength) classes are distinguished: UV-C, UV-B and UV-A. The impact of UV radiation on living organisms depends on its wavelength, the shorter the more dangerous. UV-C is the most dangerous wavelength range, but almost completely filtered by the ozone layer. UV-B (wavelengths $280-315 \mathrm{~nm}$ ) is of the most concern due to ozone layer depletion, while UV-A is not absorbed by ozone.

Depending on duration and intensity of exposure to UV-B, impacts on human health are suspected to include skin cancer, cataracts, sun burn, increased skin cell ageing, immune system diseases, headaches, burning eyes and irritation to the respiratory passages. Ecosystem effects are linked to epidermal damage to animals (observed e.g. in whales), and radiation damage to the photosynthetic organs of plants causing reduced photosynthesis, leading to lower yields and crop quality in agricultural produce and loss of phytoplankton, the primary producers of aquatic food chains, particularly in the polar oceans. Additionally, UV-B accelerates the generation of photochemical smog, thereby stimulating the production of tropospheric ozone, which is a harmful pollutant (see Sect. 10.10). 


\subsubsection{Environmental Mechanism}

Stratospheric ozone concentrations result from a balance between $\mathrm{O}_{3}$ formation and destruction under the influence of solar (UV) radiation, temperature and the presence of other chemicals. The annual cycle of ozone destruction over the poles develops under the presence of several influencing factors with its intensity directly depending on their combined intensity: (1) meteorological factors (i.e. strong stratospheric winds and low temperature) and (2) the presence of ozone depleting chemicals.

Meteorological factors involve the formation of the "polar vortex", a circumpolar stratospheric wind phenomenon, in the polar night during the polar winter, when almost no sunlight reaches the pole. This vortex isolates the air in polar latitudes from the rest of Earth's atmosphere, preventing ozone and other molecules from entering. As the darkness continues, the air inside the polar vortex gets very cold, with temperatures dropping below $-80{ }^{\circ} \mathrm{C}$. At such temperatures a special type of clouds, called Polar Stratospheric Clouds (PSC), begins to form. Unlike tropospheric clouds, these are not primarily constituted of water droplets, but of tri-hydrated nitric acid particles, which can form larger ice particles containing dissolved nitric acid in their core as temperature continues to drop. The presence of PSC is crucial for the accelerated ozone depletion over the polar regions because they provide a solid phase in the otherwise extremely clean stratospheric air on which the ozone-degrading processes occur much more efficiently.

Chemical factors involve the presence of chlorine and bromine compounds in the atmosphere as important contributors to the destruction of ozone. The majority of the chlorine compounds and half of the bromine compounds that reach the stratosphere stem from human activities.

Due to their extreme stability, CFCs are not degraded in the troposphere but slowly (over years) transported into the stratosphere. Here, they are broken down into reactive chlorine radicals under the influence of the very energy-rich UV radiation at the upper layers of the ozone layer. One chlorine atom can destroy very high numbers of ozone molecules, before it is eventually inactivated through reaction with nitrogen oxides or methane present in the stratosphere. The degradation and inactivation scheme is illustrated in a simplified form for a CFC molecule in Fig. 10.11.

When they are isolated in the polar vortex and in the presence of PSC, these stable chlorine and bromine forms come into contact with heterogeneous phases (gas/liquid or gas/solid) on the surface of the particles forming the PSC, which breaks them down and release the activated free chlorine and bromine, known as "active"/ozone depleting substances (ODS). These reactions are very fast and, as explained, strongly enhanced by the presence of PSC, a phenomenon which was neglected before the discovery of the 'ozone hole'.

While this describes the fate mechanism leading to stratospheric ozone reduction, Fig. 10.12 shows the impact pathway leading to ozone depletion in the 


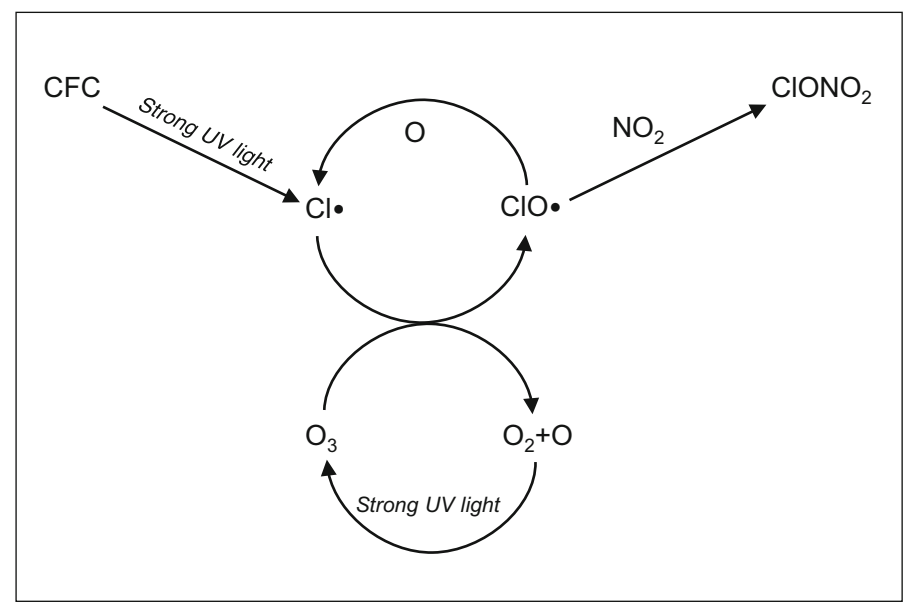

Fig. 10.11 Degradation of ozone catalysed by chlorine in the stratosphere (simplified)

Fig. 10.12 Impact pathway for stratospheric ozone depletion

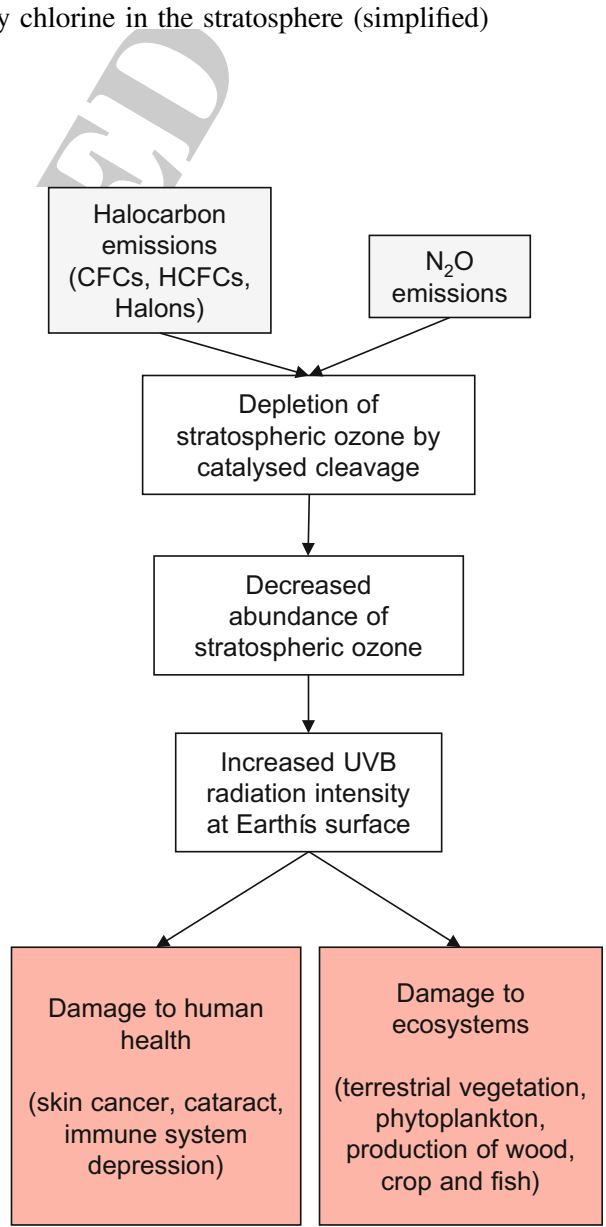


stratosphere from man-made emissions of long-lived halocarbons and nitrous oxide as used by most LCIA methods.

The midpoint indicator used without exception in all LCIA methods to calculate characterisation factors is the Ozone Depletion Potential (ODP). In a similar manner as the Global Warming Potential (GWP), it evaluates the potential of a chemical to destroy the ozone layer based on a model from the World Meteorological Organization (WMO 2014). The ODP essentially expresses the global reduction in stratospheric $\mathrm{O}_{3}$ concentration $\mathrm{C}_{\mathrm{O}_{3}}$ due to an ozone depleting substance $i$ relative to the global reduction of stratospheric $\mathrm{O}_{3}$ concentration $\mathrm{C}_{\mathrm{O}_{3}}$ due to $1 \mathrm{~kg}$ of CFC-11 $\left(\mathrm{CFCl}_{3}\right)$, and is hence expressed in CFC-11 equivalents:

$$
\mathrm{ODP}_{i}=\frac{\Delta C_{\mathrm{O}_{3}}(i)}{\Delta C_{\mathrm{O}_{3}}(\mathrm{CFC}-11)}
$$

\subsubsection{Emissions and Main Sources}

The halogen compounds in the stratosphere are mostly originating from very stable industrial halocarbon gases used as solvents or refrigerants (the chlorinated CFCs or freons), or fire extinguishers (the brominated halons). Groups of anthropogenic ODS are: bromochloromethanes (BCM), chlorofluorocarbons (CFCs), carbon tetrachloride, hydrobromofluorocarbons (HBFCs), hydrochlorofluorocarbons (HCFCs), tetrachloromethane, 1,1,1-trichloromethane, methyl bromide, methyl chloride and halons. The main uses of ODS during the last century were: fire extinguishing systems (halon), plastic foams, propellant gas in spray cans, fumigate and pesticides (methyl bromide), metered-dose inhalers (MDIs), refrigeration and air-conditioning and solvent degreasing.

Natural ozone depleting substances are $\mathrm{CH}_{4}, \mathrm{~N}_{2} \mathrm{O}, \mathrm{H}_{2} \mathrm{O}$ and halogenated substances with sufficient stability and/or release rates to allow them to reach the stratosphere. All ozone depleting substances have two common characteristics, being:

- Chemically very stable in the lower atmosphere.

- Capable of releasing chloride or bromide under UV radiation (photodissociation).

The phasing-out of production and use of the concerned substances has been successfully enforced under the Montreal protocol, which was signed in 1987 and led to phasing-out of consumption and production of ODS by 1996 in developed countries and by 2010 in developing countries. If continuously respected, this effort should lead to the cessation of the annual appearance of the 'ozone hole' around 2070, the delay being due to the facts that (1) we are still emitting decreasing amounts of relevant substances (mostly during the end-of-life treatment of old refrigeration and air-conditioning systems) and (2) they are very persistent and may 
take decades to reach the poles and hence continue their adverse effects for a prolonged time. When significant emissions or dominating impacts of ODS are observed in LCIs or LCA results nowadays, it is likely because the data originate from references before the phase-out and hence it is most likely an artefact due to obsolete data, unless the end-of-life treatment of old refrigeration and air-conditioning systems are an important component of the LCA.

\subsubsection{Existing Characterisation Models}

Without any exception, all existing LCIA methods use the ODP as midpoint indicator (although not all of them have the most recent version). For endpoint characterisation, different midpoint-to-endpoint models are applied that relate ozone depletion to increased UV radiation and ultimately to skin cancer and cataract in humans. All endpoint LCIA methods characterise impacts on human health, but only the Japanese method LIME additionally considers impacts on Net Primary Productivity (NPP) for coniferous forests, agriculture (soybean, rice, green pea, mustard) and phytoplankton at high latitudes. For further details see Chap. 40 and Hauschild and Huijbregts (2015).

\subsection{Acidification}

\subsubsection{Problem}

During the 1980s and 90s, the effects of acidification of the environment became clearly visible in the form of a pronounced lack of health especially among conifers in many forests in Europe and the USA, resulting locally in forest decline, leading to accelerated clearing of whole forests. Clear acidic lakes without fish go right back to the beginning of the twentieth century, occurring locally for example in Norway and Sweden as a result of human activities, but the extent of the problem increased dramatically in more recent times, and during the 1990s there was serious acidification in more than 10,000 Scandinavian lakes. Metals, surface coatings and mineral building materials exposed to wind and weather are crumbling and disintegrating at a rate which is unparalleled in history, with consequent major socio-economic costs and loss of irreplaceable historic monuments in many parts of the industrialised world.

The acidification problems were one of the main environmental concerns in Europe and North America in the 1980s and 90s but through targeted regulation of the main sources in the energy, industry and transportation sectors followed by liming to restore the $\mathrm{pH}$ of the natural soils and waters, it is no longer a major concern in these regions. In China, however, acidification impacts are dramatic in 
some areas due to the extensive use of coal-fired power generation using sulphur-rich coal.

\subsubsection{Environmental Mechanism}

Acidification of soil or aquatic ecosystems can be defined as an impact which leads to a fall in the system's acid neutralising capacity (ANC), i.e. a reduction in the quantity of substances in the system which are able to neutralise hydrogen ions added to the system.

ANC can be reduced by:

1. Addition of hydrogen ions, which displace other cations which can then be leached out of the system

2. Uptake of cations in plants or other biomass which is collected and removed from the system

Particularly the former is relevant for acidification impacts in LCA. Acidification occurs naturally over time, but it is greatly increased by man-made input of hydrogen ions to soil and vegetation. The main source is air-borne emissions of gases that release hydrogen when they are degraded in the atmosphere or after deposition to soil, vegetation or water. Deposition is increased during precipitation events where the gases are dissolved in water and come down with rain, which can be rather acidic with $\mathrm{pH}$ values down to 3-4 in cases of strong air pollution ("acid rain").

The most important acidifying man-made compounds are:

Sulphur oxides, $\mathrm{SO}_{2}$ and $\mathrm{SO}_{3}$ (or jointly $\mathrm{SO}_{x}$ ), the acidic anhydrides of sulphurous acid $\mathrm{H}_{2} \mathrm{SO}_{3}$ and sulphuric acid $\mathrm{H}_{2} \mathrm{SO}_{4}$, respectively, meaning that upon absorption of water from the atmosphere they form these very strong acids which both release two hydrogen ions when deposited:

$$
\begin{aligned}
& \mathrm{SO}_{2}+\mathrm{H}_{2} \mathrm{O} \rightarrow \mathrm{H}_{2} \mathrm{SO}_{3} \rightarrow 2 \mathrm{H}^{+}+\mathrm{SO}_{3}{ }^{2-} \\
& \mathrm{SO}_{3}+\mathrm{H}_{2} \mathrm{O} \rightarrow \mathrm{H}_{2} \mathrm{SO}_{3} \rightarrow 2 \mathrm{H}^{+}+\mathrm{SO}_{4}{ }^{2-}
\end{aligned}
$$

Nitrogen oxides, $\mathrm{NO}$ and $\mathrm{NO}_{2}$ (or jointly $\mathrm{NO}_{x}$ ) that are also acidic anhydrides as they can be converted to nitric and nitrous acids by oxidation in the troposphere. $\mathrm{NO}$ is oxidised to $\mathrm{NO}_{2}$ primarily by reaction with ozone (see Sect. 10.10):

$$
\mathrm{NO}+\mathrm{O}_{3} \rightarrow \mathrm{NO}_{2}+\mathrm{O}_{2}
$$

$\mathrm{NO}_{2}$ can be oxidised to nitric acid, $\mathrm{HNO}_{3}$ or $\mathrm{HONO}_{2}$ : 


$$
\mathrm{NO}_{2}+\mathrm{OH}+\mathrm{M} \rightarrow \mathrm{HONO}_{2}+\mathrm{M}
$$

where $\mathrm{OH}$ is hydroxyl radical present in the atmosphere and $\mathrm{M}$ is an inactive body which can remove surplus energy.

Ammonia, which is in itself a base (absorbing hydrogen ions via the reaction $\mathrm{NH}_{3}+\mathrm{H}^{+} \rightarrow \mathrm{NH}_{4}{ }^{+}$), but upon complete mineralisation through nitrite, $\mathrm{NO}_{2}{ }^{+}$, to nitrate, $\mathrm{NO}_{3}{ }^{-}$releases net one proton:

$$
\mathrm{NH}_{3}+2 \mathrm{O}_{2} \rightarrow \mathrm{H}^{+}+\mathrm{NO}_{3}{ }^{-}+\mathrm{H}_{2} \mathrm{O}
$$

Strong acids like hydrochloric acid, $\mathrm{HCl}$ or sulphuric acid, $\mathrm{H}_{2} \mathrm{SO}_{4}$, which release their content of hydrogen ions as soon as they are dissolved in water and thus also are strongly acidifying.

Because of their high water solubility, the atmospheric residence time of these acidifying substances is limited to a few days, and therefore acidification is a regional effect with its extent limited to the region around the point of emission.

When acidifying compounds deposit on plant leaves or needles, they can damage these vital plant organs and through this damage the plants. When the acidifying compounds reach the soil, protons are released in the soil where they may lower the $\mathrm{pH}$ of the soil water and cause release of metal ions bound in the soil. Some of these metals are toxic to the plants in the soil, others are essential for plant growth, but after their release, they wash out, and the availability of these metals to plants may then become limiting for plant growth. The result is stress on the plants through root and leaf damage and after prolonged exposure the plants may die as a direct consequence of this or through diseases or parasites that benefit from the weakened constitution of the plant. Lakes are also exposed to the acidification, in particular through the acidified soil water leaching to the lake. When the $\mathrm{pH}$ of a lake drops, it affects the availability of carbon in the water as $\mathrm{HCO}_{3}{ }^{-}$, which is the dominating form around neutral $\mathrm{pH}$, is converted to dissolved $\mathrm{CO}_{2}$. The solubility of toxic metals is increased, in particular aluminium which may precipitate on the gills of fish at $\mathrm{pH}$ 5. The phytoplankton and macrophyte flora gradually change and also the fauna is affected. Humic acids that give the lakewater a brown colour are precipitated, and the acidified lakes appear clear and blue.

The sensitivity to acidification is strongly influenced by the geology and nature of the soil. Calcareous soils with a high content of calcium carbonate are well buffered meaning that they will resist the change in $\mathrm{pH}$ by neutralising the input of hydrogen ions with the basic carbonate ions:

$$
\begin{gathered}
\mathrm{H}^{+}+\mathrm{CaCO}_{3} \rightarrow \mathrm{Ca}^{2+}+\mathrm{HCO}_{3}{ }^{-} \\
\mathrm{H}^{+}+\mathrm{HCO}_{3}{ }^{-} \rightarrow \mathrm{H}_{2} \mathrm{O}+\mathrm{CO}_{2}
\end{gathered}
$$

As long as there is calcium carbonate in the soil, it will thus not be acidified. 
Soils that are rich in clay are also resistant to acidification through their ability to adsorb the protons on clay mineral surfaces under release of metal ions, while sandy soils are more sensitive to acidification. The sensitivity of an ecosystem towards acidification can be described by its critical load-“"A quantitative estimate of an exposure to one or more pollutants below which significant harmful effects on specified sensitive elements of the environment do not occur according to present knowledge" (Nilsson and Grennfelt 1988). Critical loads are high in calcareous regions like the Mediterranean and low in e.g. granite rock regions like most of Scandinavia.

Incorporating the environmental mechanism described above, the impact pathway of acidification is illustrated in Fig. 10.13.

Oceanic acidification is the process of dissolution of $\mathrm{CO}_{2}$ into seawater leading to a slight lowering of the $\mathrm{pH}$ in the open oceans as a consequence of increasing concentrations of $\mathrm{CO}_{2}$ in the atmosphere. Dissolution of $\mathrm{CO}_{2}$ in water generates carbonic acid, a rather weak acid (think soda water), which releases protons according to

$$
\mathrm{CO}_{2}+\mathrm{H}_{2} \mathrm{O} \rightarrow \mathrm{H}_{2} \mathrm{CO}_{3} \rightarrow \mathrm{HCO}_{3}^{-}+\mathrm{H}^{+}
$$

The slightly lowered $\mathrm{pH}$ is deleterious to coral reefs, which should be included in endpoint characterisation. $\mathrm{CO}_{2}$ is the only important contributor to oceanic acidification and inclusion of this impact category on midpoint level therefore offers little additional information to the LCIA that already considers climate change, we will hence not discuss it further here.

\subsubsection{Emissions and Main Sources}

Sulphur dioxides and nitrogen oxides are the man-made emissions that contribute the most to acidification. Historically metal smelters of the mining industry have been strong sources of local acidification with large localised emissions of sulphur oxides. Today, the main sources of both $\mathrm{SO}_{x}$ and $\mathrm{NO}_{x}$ are combustion processes in thermal power plants, combustion engines, waste incinerators and decentralised furnaces. For sulphur oxides, the level of emissions depends on the sulphur content of the fuels. Since nitrogen is abundant in the atmosphere and hence in all combustion processes using air, emissions of nitrogen oxides are mainly determined by conditions of the combustion process and possible treatment of the flue gases through catalysers and filters. As response to the serious problems with acidification in Europe and North America in previous times, regulation now ensures that sulphur content is removed from the fuels, that important combustion activities like thermal power plants and waste incinerators have an efficient neutralisation of the flue gases before they are released, and that combustion engines have catalysers lowering the $\mathrm{NO}_{x}$ content of the exhaust gases. 


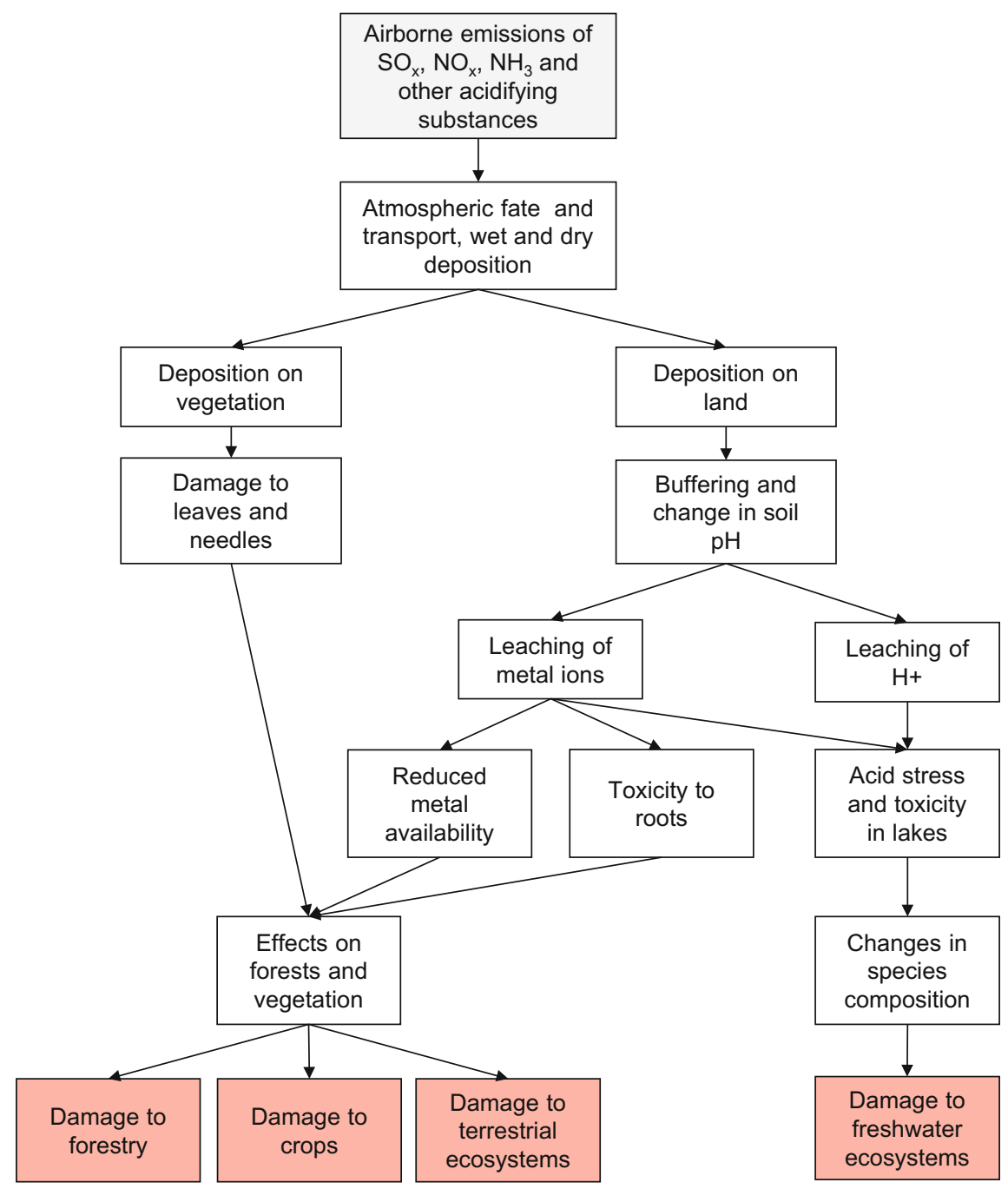

Fig. 10.13 Impact pathway for acidification

Ammonia is also an important contributor to acidification in some regions and the main sources are all related to agriculture using $\mathrm{NH}_{3}$ as a fertiliser, and to animal husbandry, in particular pig and chicken farms, with ammonia emissions from stables and dispersion of manure.

Mineral acids like $\mathrm{HCl}$ and $\mathrm{H}_{2} \mathrm{SO}_{4}$ rarely appear as elementary flows in life cycle inventories but they may be emitted from some industrial processes and also from waste incinerators with inefficient flue gas treatment. 


\subsubsection{Existing Characterisation Models}

The acidification potential depends both on the potency of the emitted gas and on the sensitivity of the receiving environment in terms of buffering capacity of the soils and sensitivity of the ecosystems to acidification as expressed by their critical load. While the difference between the contributing gases is modest-within a factor 5-10 across substances, the difference between sensitivities in different locations can be several orders of magnitudes depending on the geology and soil characteristics. Early characterisation models were site-generic and only incorporated the difference in ability to release protons, but newer models incorporate more and more of the cause-effect chain in Fig. 10.13 and model e.g. the area of ecosystem in the deposition area that becomes exposed above its critical load. This requires a site-dependent LCIA approach where the characterisation factor is determined not just per emitted substance but also per emission location. Characterisation factors may be expressed as absolute values or as an equivalent emission of a reference substance which in that case is usually $\mathrm{SO}_{2}$. For further details see Chap. 40 and Hauschild and Huijbregts (2015).

\subsection{Eutrophication}

\subsubsection{Problem}

Nutrients occur naturally in the environment, where they are a fundamental precondition for the existence of life. The species composition and productivity of different ecosystems reflect the availability of nutrients, and natural differences in the availability of nitrogen and phosphorus are thus one of the reasons for the existing multiplicity of species and of different types of ecosystems. Ecosystems are dynamic, and if they are affected by a changed availability of nutrients, they simply adapt to a new balance with their surroundings. Originally, eutrophication of aquatic environments, such as rivers or lakes, describes its eutrophic character (from the Greek word "eu"-good, true-and "trophein"-feed), meaning nutrient-rich. From the 1970s the term was used to describe the slow suffocation of large lakes. It now has a meaning close to dystrophic. An aquatic ecosystem in strong imbalance is named hypertrophic, when close to a natural equilibrium it is called mesotrophic, and when healthy it is called oligotrophic.

The perhaps most prominent effect of eutrophication in lakes, rivers and the coastal sea are lower water quality including low visibility or for stronger situations massive amounts of algae in the surface layers of those waters. Eutrophication essentially describes the enrichment of the aquatic environment with nutrient salts leading to an increased biomass production of planktonic algae, gelatinous zooplankton and higher aquatic plants, which results in a degradation of (organoleptic) water quality (e.g. appearance, colour, smell, taste) and an altered species 
composition of the ecosystem. It may also lead to the development of toxic phytoplankton, dynophysis, cyanobacteria or blue-green algae. When the algae die, they sink to the bottom where they are degraded under oxygen consumption. As a consequence, the concentration of dissolved oxygen decreases (hypoxia), which results in biodiversity loss (flora and fauna). Ultimately, if the process is not stopped, this will turn a lake into a swamp, that will become grassland and forest. This process occurs naturally but over a much longer time horizon.

For terrestrial systems, the most significant environmental problem in relation to nitrogen compound loading is changes in the function and species composition of nitrogen-poor (and $\mathrm{N}$-limited) ecosystems in heathlands, dune vegetation, commons and raised bogs as a result of the atmospheric deposition of nitrogen compounds. Forestry and agriculture may also be affected by reduced yields via damage to forests and crops. This section however focuses on aquatic eutrophication.

\subsubsection{Environmental Mechanism}

The food chain in aquatic ecosystems can be distinguished into three trophic levels: primary producers (algae and plants producing biomass via photosynthesis), primary consumers (species consuming algae and plants, the vegetarians) and secondary consumers (species consuming primary consumers, the carnivores). In addition to sunlight, growth of primary producers (algae and higher plants) requires all of the elements which enter into their anabolism (i.e. their synthesis of the molecules which constitute the organisms' cells). A formula for the average composition of an aquatic organism is $\mathrm{C}_{106} \mathrm{H}_{263} \mathrm{O}_{110} \mathrm{~N}_{16} \mathrm{P}$ (Stumm and Morgan 1981). Apart from the elements represented in this formula, minor quantities of a large number of other elements are required, e.g. potassium, magnesium, calcium, iron, manganese, copper, silicon and boron (Salisbury and Ross 1978). In principle, the availability of any of these elements can determine the potential extent of the growth of the primary producers in a given system. The elements entering in greatest quantities into the primary producers (as in all other living organisms) are carbon, $\mathrm{C}$, hydrogen, $\mathrm{H}$ and oxygen, $\mathrm{O}$. The availability of water can limit growth in terrestrial plants, but the availability of one of the three basic elements is rarely a limiting factor in the growth of primary producers.

The other elements which enter into the construction of the primary producers are nutrients, as the availability of these elements in sufficient quantities is necessary to ensure growth. The nutrients are classified as macronutrients $(>1000 \mu \mathrm{g} / \mathrm{g}$ dry matter in plants) and micronutrients ( $<100 \mu \mathrm{g} / \mathrm{g}$ dry matter in plants) (Salisbury and Ross 1978). In rare cases, growth is limited by the availability of one of the micronutrients, but very small quantities of these elements are required by the primary producers, and these elements are therefore limiting only on very poor soils. Of the macronutrients, sulphur is added to all ecosystems in fair quantities in most of the industrialised world by the atmospheric deposition of sulphur com- 
pounds from flue gases deriving from energy production via fossil fuels. Calcium, potassium and magnesium occur in lime and clay, respectively, which exist in large quantities in soils.

In practice, one of the two last macronutrients, nitrogen and phosphorus, is therefore almost always the limiting element for the growth of primary producers, and it is therefore reasonable to regard only the elements nitrogen and phosphorus as contributors to nutrient enrichment. In many lakes, phosphorus deficiency, or a combination of nitrogen and phosphorus deficiencies, is typically limiting growth, and their addition promotes algal growth. In coastal waters and seas, nitrogen is often the limiting nutrient. Substances which contain nitrogen or phosphorus in a biologically available form are therefore classified as potential contributors to nutrient enrichment. As is evident from the formula for the average composition of aquatic organisms, the ratio of nitrogen to phosphorus is of the order of 16. If the concentration of bioavailable nitrogen is significantly more than 16 times the concentration of bioavailable phosphorus in an ecosystem, it is thus reasonable to assume that phosphorus is the limiting nutrient, and vice versa. Since most of the atmosphere consists of free nitrogen, $\mathrm{N}_{2}$, further addition of $\mathrm{N}_{2}$ will not have any effect, and it is also not directly bioavailable. $\mathrm{N}_{2}$ is therefore not classified as contributing to nutrient enrichment.

For aquatic eutrophication, the starting point of the cause-effect chain is the emissions of a compound containing either Nitrogen $(\mathrm{N})$ or Phosphor $(\mathrm{P})$. Increased availability of nutrients will primarily increase the growth of algae and plants, especially in summer with abundant sunlight. This algae growth is visible as rivers, lakes or coastal waters turn turbid in summer. Eventually, the algae will sink to the bottom where they are decomposed by degraders like bacteria under consumption of oxygen in the bottom layer. With the sunlight being increasingly blocked from reaching deeper water layers, the build-up of a temperature gradient causes stratification in deep lakes and some coastal waters in the summer months. In the marine environment stratification is determined by density differences between salt water flowing in from the sea and brackish water flowing out from river deltas and fjords. Such stratification prevents effective mixing of the water column. If fresh oxygen-rich water from the surface does not find its way to the bottom layers, the oxygen concentration near the bottom will gradually be reduced until the bottom-dwelling organisms move away or die. As the oxygen concentration approaches zero, poisonous substances such as hydrogen sulphide, $\mathrm{H}_{2} \mathrm{~S}$, are formed in the sediments, where they accumulate in gas pockets which, on their release, kill those organisms exposed to them.

The main cause-effect chain as shown in Fig. 10.14 can be summarised as:

\section{- Emission of $\mathrm{N}$ or $\mathrm{P}$}

- Growth and blooming of algae and higher plants increases

- Sunlight no longer reaches lower water layers, which creates a temperature gradient with increasing depth

- This supports a stable stratification of water layers reducing the transport of fresh oxygen-rich surface water to deeper layers 
Terrestrial eutrophication

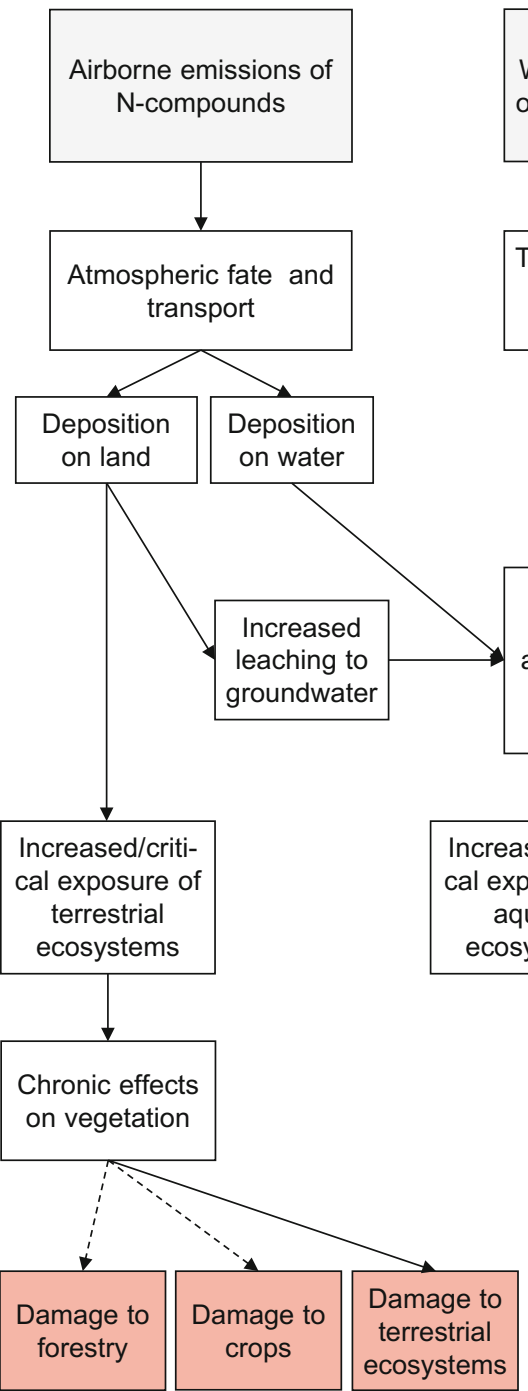

\section{Aquatic eutrophication}

Waterborne emissions of $\mathrm{N}$ and $\mathrm{P}$ compounds

Transport/removal of $\mathrm{N}$ and $P$ in water compartments
Waterborne emissions

of dissolved organic compounds (DOC, COD, BOD)

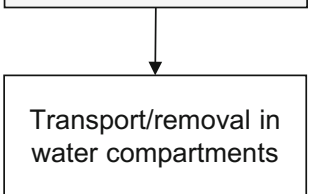

Fig. 10.14 Impact pathways for terrestrial and aquatic (freshwater and marine) eutrophication [adapted from EC-JRC (2011)]

- Oxygen is steadily depleted in bottom layers, which leads to suffocation of bottom-dwelling species and fish

- This is additionally accelerated by the oxygen consuming decomposition of the dead species and sedimented dead algae

- The medium becomes hypoxic and finally anoxic, favouring the formation of reducing compounds and noxious gases (mercaptans, methane) 
In a tripartite division of environmental impact categories into global, regional and local, eutrophication is considered a local to regional impact. As a consequence of the above explanations, impact potentials are highly dependent on local conditions, e.g. whether the recipient of the emission will support the requisite conversion of the emission (e.g. mineralisation of organic nitrogenous compounds), or whether the recipient is limited in nitrogen or phosphorus, while both elements are always considered potential contributors to eutrophication.

The calculation of characterisation factors for a nutrient enriching substance consists of an assessment of the number of moles of nitrogen or phosphorus which can be released into the environment from one mole of the substance emitted. This can be expressed in the form of two nutrient enrichment equivalents, as $\mathrm{kg}$ $\mathrm{N}$-equivalents and kg P-equivalents. The possible consequences of eutrophication are often irrespective of whether nitrogen or phosphorus is the causing agent. In some situations it can therefore be desirable to reduce the complexity of the results of the environmental assessment by expressing eutrophication as one equivalent, so that the contributions for nitrogen and phosphorus are aggregated. In this case the impact potential may also be expressed as an equivalent emission of a reference substance (e.g. $\mathrm{NO}_{3}{ }^{-}$one of the most important nutrient enrichment substances). Aggregation of $\mathrm{N}$ and $\mathrm{P}$ potentials requires an assumption concerning the magnitude of the ratio N/P between the two elements in living organisms. As explained above a molar ratio of 16 can be used for nitrogen:phosphorus in living material. One mole of phosphorus (in an area where the availability of phosphorus limits growth) therefore contributes as much to eutrophicationas $16 \mathrm{~mol}$ of nitrogen (in an area where the availability of nitrogen limits growth). The aggregate nutrient enrichment potential for nitrogenous substances is then calculated as the emission's $\mathrm{N}$ potential multiplied by the gram molecular weight of the reference substance (e.g. $\mathrm{NO}_{3}{ }^{-}$of $62.00 \mathrm{~g} / \mathrm{mol}$ ). The $\mathrm{P}$ potential for phosphorous-containing substances is multiplied by 16 times the gram molecular weight of the reference substance.

The primary receiving compartment for agricultural emissions is mainly freshwater where some of the nitrogen may be removed on the way to the marine systems by denitrification in rivers and lakes converting the nitrogen into $\mathrm{N}_{2}$ which is released to the atmosphere. Loading of freshwater with nitrogen is thus greater than the quantity conveyed to the marine areas via rivers and streams. Phosphorous compounds do not undergo this kind of conversion but phosphate forms insoluble salts with many metals and this may lead to some removal through accumulation of phosphorus in lake sediments. Phosphorus accumulated in the sediments of rivers and streams during drier periods may later be washed out into the marine environment when the water flow increases, e.g. after a thunderstorm.

\subsubsection{Emissions and Main Sources}

Due to the use of inorganic fertilisers and manure, agriculture is a significant source of phosphate and nitrogen emissions in the form of nitrates, affecting groundwater 
via percolation and surface water via runoff and leaching processes, and of ammonia emitted to air and deposited on land nearby. Oxides of nitrogen may be emitted from incineration processes. Point sources in the form of wastewater treatment plants for households (e.g. from polyphosphates in detergents) and industry as well as fish farming are important sources of phosphorus and nitrates. Apart from man-made emissions, natural sources include leaching and runoff of nitrogen and phosphates. The natural addition of nutrients to terrestrial areas is believed to consist mainly of atmospheric deposition of oxides of nitrogen and ammonia while some natural plant species also possess the ability to fixate atmospheric nitrogen.

Emissions of organic materials can lead to oxygen consumption by bacteria degrading this organic matter and thus contributing to oxygen depletion similarly to what is observed as a result of the nutrient enrichment of lakes and coastal waters. However, this is a primary effect and is strictly speaking not part of the nutrient enrichment mechanism. Therefore, emissions of BOD (biological oxygen demand - substances which consume oxygen on degradation) or COD (chemical oxygen demand) may additionally be characterised by some LCIA methods considering oxygen depletion (hypoxia) in water as a common midpoint for both mechanisms. Most LCIA methods are currently based on the N/P ratio and typically do not classify $\mathrm{BOD} / \mathrm{COD}$ as contributing to nutrient enrichment and thus eutrophication. In large parts of the industrialised world organic matter emissions are only of local significance in watercourses and for occasional emissions of untreated effluent.

\subsubsection{Existing Characterisation Models}

The essential evolutions during the last decade were related to improved fate modelling, distinguishing P-limited (freshwater) and N-limited (marine) ecosystems, introduction of a midpoint effect factor in the more recent methods, and characterisation models becoming global and spatially more detailed. Midpoint LCIA methods usually propose units in $\mathrm{P}$ - and $\mathrm{N}$-equivalents such as $\mathrm{kg}$ P-eq or $\mathrm{kg} \mathrm{PO}_{4}{ }^{3-}{ }^{-}$-eq and $\mathrm{kg} \mathrm{N}$-eq or $\mathrm{NO}_{3}{ }^{-}$-eq. For endpoint characterisation most models use Potentially Disappeared Fraction of species (PDF) in $\left[\mathrm{m}^{2}\right.$ years], except LIME which uses Net Primary Productivity (NPP) loss. For further details see Chap. 40 and Hauschild and Huijbregts (2015).

\subsection{Photochemical Ozone Formation}

This impact category appears under a number of different names in the various LCIA methods: (tropospheric) ozone formation, photochemical ozone formation or creation, photo oxidant formation, photosmog or summer smog. There are minor 
differences, but in essence they all address the impacts from ozone and other reactive oxygen compounds formed as secondary contaminants in the troposphere by the oxidation of the primary contaminants volatile organic compounds (VOC), or carbon monoxide in the presence of nitrogen oxides $\left(\mathrm{NO}_{x}\right)$ under the influence of light. VOCs are here defined as organic compounds with a boiling point below $250{ }^{\circ} \mathrm{C}$ (WHO 1989). $\mathrm{NO}_{x}$ is a joint name for the nitrogen monoxide $\mathrm{NO}$ and nitrogen dioxide $\mathrm{NO}_{2}$.

\subsubsection{Problem}

The negative impacts from the photochemically generated pollutants are due to their reactive nature which enables them to oxidise organic molecules in exposed surfaces. Impacts on humans arise when the ozone and other reactive oxygen compounds, which are formed in the process, are inhaled and come into contact with the surface of the respiratory tract, where they damage tissue and cause respiratory diseases. Impacts on vegetation arise when the reactive compounds attack the surfaces of plants or enter plant leaves and cause oxidative damage on their photosynthetic organs. Impacts on man-made materials are caused by oxidation and damage many types of organic materials which are exposed to ambient air. It is thus not the VOCs per se which cause the environmental problems associated with photochemical ozone formation, but the products of their transformation in the troposphere which is the lower stratum of the atmosphere, from the surface of the earth to the tropopause $8-17 \mathrm{~km}$ above us. Direct toxic effects on humans from VOCs are treated separately in the impact category human toxicity. Apart from a general increase in the tropospheric ozone concentration, photochemical ozone formation may cause smog-episodes on a more local scale in and around cities with a combination of large emissions and the right meteorological conditions. During smog-episodes, the concentrations of ozone and other photooxidants reach extreme levels causing immediate damage to human health.

\subsubsection{Environmental Mechanism}

The photochemical formation of ozone and other reactive oxygen compounds in the troposphere from emissions of VOCs and $\mathrm{NO}_{x}$ follows rather complex reaction schemes that depend on the nature of the specific organic compound. A simplified presentation of the fundamental elements of the schemes is given in Fig. 10.15 and can be summarised as:

1. VOCs (written as $\mathrm{RH}$ ) or $\mathrm{CO}$ react with hydroxyl radical $\mathrm{OH}^{-}$in the troposphere and form peroxy radicals, ROO

2. The peroxy radicals oxidise $\mathrm{NO}$ to $\mathrm{NO}_{2}$ 
Fig. 10.15 Simplified presentation of the photochemical formation of ozone

3. $\mathrm{NO}_{2}$ is split by sunlight with formation of $\mathrm{NO}$ and release of free oxygen atoms 4. Free oxygen atoms react with molecular oxygen $\mathrm{O}_{2}$ to form ozone

Both VOCs and nitrogen oxides are thus needed for the photochemical ozone formation and both contribute to the formation of ozone and other oxidants. VOC and $\mathrm{NO}_{x}$ sources are very heterogeneously distributed across Europe. VOC emissions involve hundreds of different organic compounds, depending on the nature of the source and activity causing the emission. This means that at the regional level, photochemical formation of ozone is highly non-linear and dynamic with the influence of meteorological conditions and on top of this the interaction between the different VOCs from both anthropogenic and natural sources like forests, and a large number of different reaction products. A further complication arises because NO may react with the formed ozone, abstracting an oxygen atom to give oxygen and $\mathrm{NO}_{2}$. This means that depending on the conditions, $\mathrm{NO}$ may locally have a negative ozone formation potential and hence a negative characterisation factor for this impact category. Rather than a permanent removal of ozone this reaction of NO leads to a geographic displacement of the ozone formation since the $\mathrm{NO}_{2}$ thus formed can later cause ozone formation again following the scheme in Fig. 10.15, just in a different location.

The ozone formation requires the reaction between hydroxyl radical and a bond between carbon and hydrogen or another carbon atom in a VOC molecule. The relative strength of a volatile organic compound in terms of ozone formation potential per unit weight thus depends on how many such bonds it contains. The strength grows with the number of double or triple bonds and declines with the content of other elements than carbon and hydrogen. The following general ranking can be given from high to low ozone formation potential: 
1. Alkenes (decreasing with chain length) and aromatics (increasing with the degree of alkyl substitution, decreasing with the length of the chain in the substituted alkyl group)

2. Aldehydes (the strongest is formaldehyde; benzaldehyde has no or even a negative ozone formation potential)

3. Ketones

4. Alkanes (almost constant from a chain length of three carbon atoms and upwards), alcohols and esters (the more oxygen in the molecule, the weaker)

5. Halocarbons (decreasing with the degree of halogen substitution and the weight of the halogen element)

Animals and humans are mainly exposed to the photochemical oxidants through inhalation of the surrounding air, and the effects therefore appear in their respiratory organs. Ozone is detectable by its odour at a concentration of ca. $20 \mathrm{ppb}$ in pure air, but only at somewhat higher concentrations we start to see acute symptoms like increased resistance of the respiratory passages and irritation of the eyes, followed at even higher concentrations by more serious effects like oedema of the lungs, which can lead to long-term incapacity. Smog-episodes with extreme concentrations of photochemical oxidants in urban areas are known to cause increased mortality. Chronic respiratory illness may result from long-term exposure to the photochemical oxidants.

Plants rely on continuous exchange of air between their photosynthetic organs (leaves or needles) and the atmosphere to absorb the carbon dioxide which is needed for photosynthesis. Ozone and other photooxidants enter together with the air and through their oxidative properties damage the photosynthetic organelles, leading to discolouration of the leaves followed by withering of the plant. The sensitivity of the plant varies with the season and also between plant species, but considerable growth reductions are observed in areas with high ozone concentrations during the growth season. Agriculture yield losses of $10-15 \%$ have been estimated for common crop plants.

Figure 10.16 summarises the impact pathway for photochemical ozone formation linking emissions of VOCs, $\mathrm{CO}$ and $\mathrm{NO}_{x}$ to the resulting damage to the areas of protection.

\subsubsection{Emissions and Main Sources}

In some cases the emissions of individual substances are known, but in the case of oil products the emissions will often be composed of many different substances and will be specified under collective designations like VOCs or nmVOCs (non-methane VOCS, i.e. VOCs apart from methane which is typically reported separately due to its nature as a strong greenhouse gas) and sometimes also HCs (hydrocarbons), or nmHCs (non-methane hydrocarbons, i.e. hydrocarbons excluding methane). 


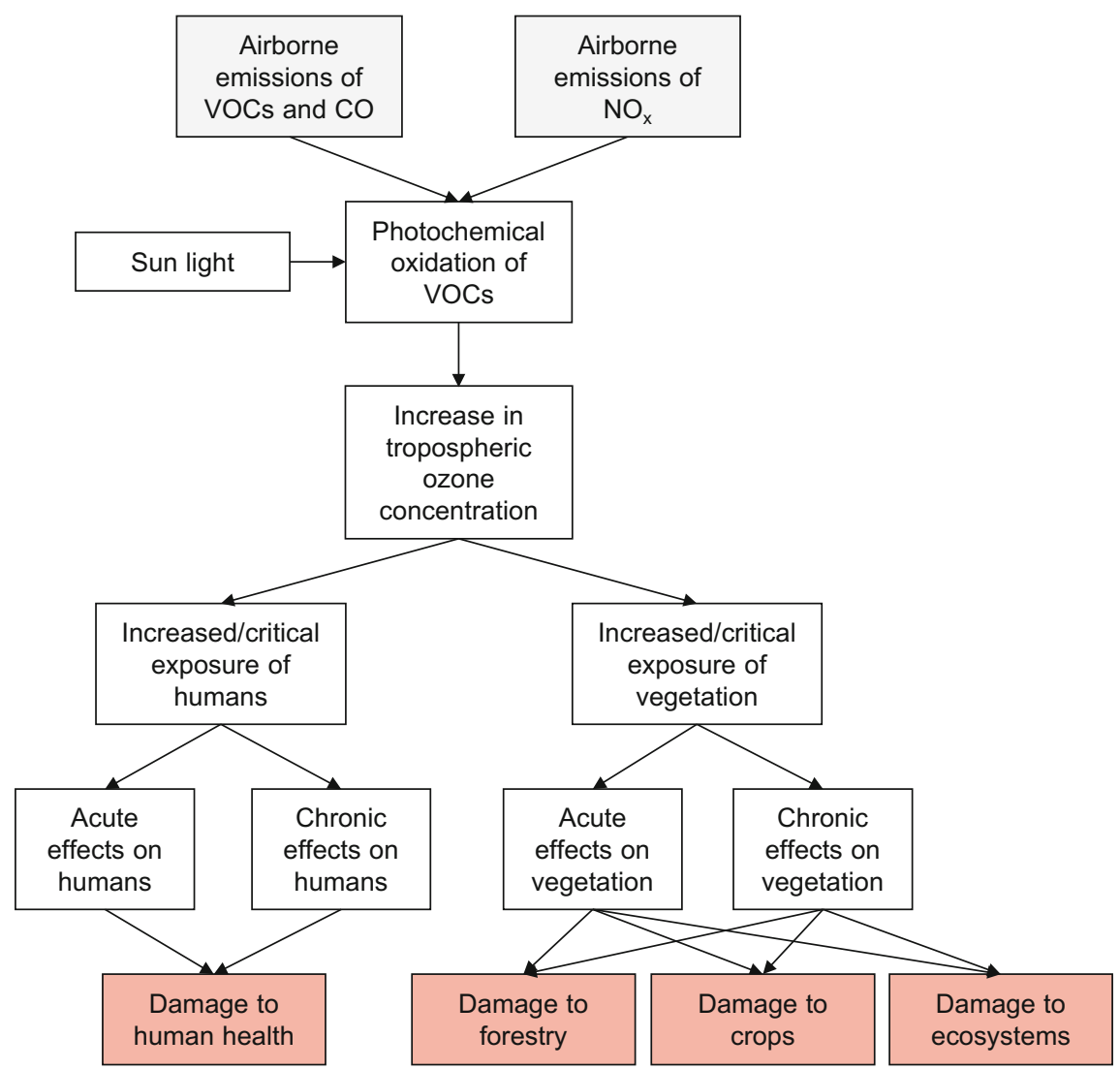

Fig. 10.16 Impact pathway for photochemical ozone formation [adapted from EC-JRC (2011)]

The most important man-made emissions of VOCs derive from road traffic and the use of organic solvents, which during 2000-2010 in Europe amounted to around $40 \%$ of the total man-made nmVOC emissions. A further $7 \%$ derives from industrial processes and 10\% are fugitive emissions (Laurent and Hauschild 2014). VOCs are also emitted in large quantities from vegetation, in particular forests, but unless a man-made manipulation of the natural system affects its emissions of VOCs, these will not be reported in an LCI and hence not dealt with in the impact assessment.Carbon monoxide is emitted from combustion processes with insufficient oxygen supply. These include road traffic and various forms of incomplete combustion of fossil fuels or biomass in stationary systems. Nitrogen oxides are also emitted from combustion processes in transport, energy- and waste incineration systems where atmospheric nitrogen is the main source of nitrogen. 


\subsubsection{Existing Characterisation Models}

The complexity of the underlying reaction schemes and the high number of individual contributing substances for which photochemical ozone formation characterisation factors must be calculated calls for simplification in the characterisation modelling. Existing characterisation models apply one of two approaches:

The first alternative is to simplify the non-linear and dynamic behaviour of the photochemical oxidation schemes by modelling one or a few typical situations in terms of meteorology, atmospheric chemistry and concomitant emissions of other air pollutants. For each individual VOC, characterisation factorsmay then be presented for each situation or in the form of a weighted average across the situations.

The second alternative is to ignore the variation between individual VOCs and concentrate on getting the spatial and temporal specificities well represented in the characterisation model. This approach leads to spatially (and possibly temporally) differentiated characterisation factors for VOCs (as a group, ignoring variation in strength between individual substances), $\mathrm{CO}$ and $\mathrm{NO}_{x}$. Often methane is treated separately from the rest of the VOCs (which are then termed non-methane VOCs or nmVOCs) due to its very low characterisation factor which really distinguishes it from the majority of the other VOCs.

The first approach is adopted in characterisation models based on the POCP (Photochemical Ozone Creation Potential) or MIR (Maximum Incremental Reactivity) concept. The second approach is adopted in regionally differentiated models which attempt to capture the non-linear nature of the ozone formation with its spatially and temporally determined differences. For further details see Chap. 40 and Hauschild and Huijbregts (2015).

\subsection{Ecotoxicity}

The contents of this section have been modified from Rosenbaum, R.K.: Ecotoxicity, appearing as Chapter 8 of Hauschild MZ and Huijbregts MAJ (eds.) (2015) LCA Compendium - The Complete World of Life Cycle Assessment—Life Cycle Impact Assessment, Springer, Heidelberg.

\subsubsection{Problem}

About 500 years ago Paracelsus stated that 'All substances are poisons; there is none which is not a poison. The right dose differentiates a poison and a remedy'. Today's toxicology science still agrees and adheres to this principle and in consequence any substance emitted may lead to toxic impacts depending on a number of driving factors: (1) emitted quantity (determined in the LCI), (2) mobility, 
(3) persistence, (4) exposure patterns and bioavailability and (5) toxicity, with the latter four considered by the characterisation factor.

This shows that toxicity is not the only parameter that determines the potential ecotoxic impact of a chemical in the environment as it first has to reach and enter a potential target organism. For example, a substance may be very toxic, but never reach any organism due to its short lifetime in the environment (e.g. rapid degradation) or because it is not sufficiently mobile to be transported to a target organism and ends up bound to soil or buried in sediment, in which case it contributes little to ecotoxic impacts. On the other hand, another substance may not be very toxic, but if it is emitted in large quantities and over prolonged periods of time or has a strong environmental persistence, it may still cause an ecotoxic impact.

Chemical emissions into the environment will affect terrestrial, freshwater, marine and aerial (i.e. flying and gliding animals) ecosystems depending on the environmental conditions of the place of emission and the characteristics of the substance emitted. They can affect natural organisms in many different ways, causing increased mortality, reduced mobility, reduced growth or reproduction rate, mutations, behavioural changes, changes in biomass or photosynthesis, etc.

\subsubsection{Environmental Mechanism}

As shown in Fig. 10.17, the environmental mechanism of ecotoxic impacts of chemicals in LCA can be divided into four consecutive steps.

1. Fate modelling estimates the increase in concentration in a given environmental medium due to an emission quantified in the life cycle inventory

2. The exposure model quantifies the chemical's bioavailability in the different media by determining the bioavailable fraction out of the total concentration

3. The effect model relates the amount available to an effect on the ecosystem. This is typically considered a midpoint indicator in LCA, as no distinction between the severity of observed effects is made (e.g. a temporary/reversible decrease in mobility and death are given the same importance)

4. Finally, the severity (or damage) model translates the effects on the ecosystem into an ecosystem population (i.e. biodiversity) change integrated over time and space

All four parts of this environmental mechanism are accounted for in the definition of the substance-specific and emission compartment-specific ecotoxicity characterisation factor $\mathrm{CF}_{\mathrm{eco}}$ :

$$
\mathrm{CF}_{\text {eco }}=\mathrm{FF} \times \mathrm{XF}_{\text {eco }} \times \mathrm{EF}_{\text {eco }} \times \mathrm{SF}_{\text {eco }}
$$

where $\mathrm{FF}$ is the fate factor, $\mathrm{XF}_{\text {eco }}$ the ecosystem exposure factor, $\mathrm{EF}_{\text {eco }}$ the ecotoxicity effect factor (midpoint effects), and $\mathrm{SF}_{\text {eco }}$ the ecosystem severity factor 


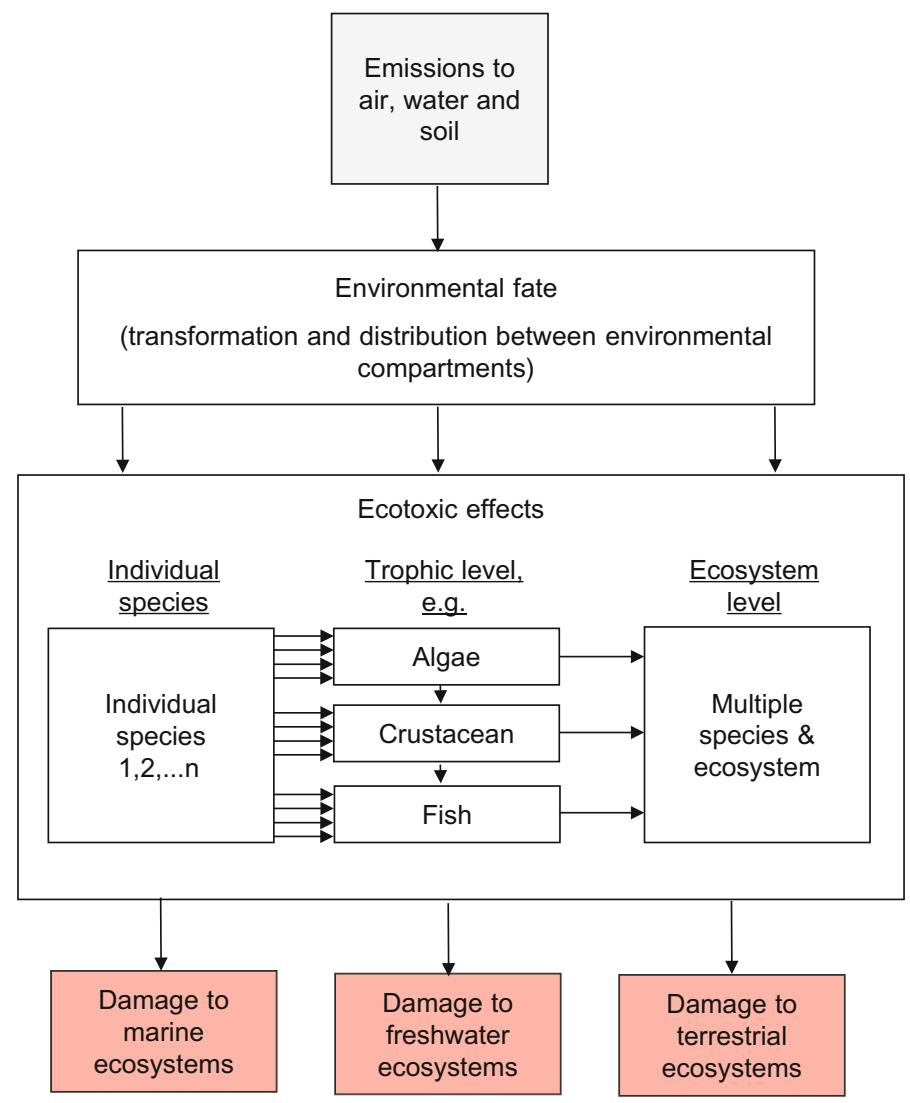

Fig. 10.17 General scheme of the Impact pathway for ecotoxicity [adapted from EC-JRC (2011)]

(endpoint effects). Each of these four elements of the environmental mechanism of ecotoxicity, and thus its characterisation factor, is described in the following sections. Some LCIA methods also directly combine $\mathrm{EF}_{\text {eco }}$ and $\mathrm{SF}_{\text {eco }}$ into a single damage factor, directly calculating an endpoint characterisation factor. For midpoint characterisation, $\mathrm{SF}_{\text {eco }}$ is simply omitted and $\mathrm{CF}_{\mathrm{eco}}$ is then the midpoint ecotoxicity characterisation factor.

A method for toxic impact assessment of chemicals in the framework of LCA must be able to cover the very large number of potentially toxic substances in the inventory in terms of available characterisation factors. It must also be based on integration of the impact over time and space as LCI data are typically not spatially and/or temporally differentiated, and the characterisation factor must relate to a mass flow and not require any information about concentrations of the substance as this information is not available in the LCI. To be compatible with the effect model, the fate model must translate chemical emissions calculated in the life cycle 
inventory into an increase in concentration in the relevant medium. In the characterisation modelling this leads to the use of fate models assuming steady-state conditions.

The fate model predicts the chemical behaviour/distribution in the environment accounting for multimedia (i.e. between environmental media and compartments) and spatial (i.e. between different zones but within the same compartment or medium) transport between environmental compartments (e.g. air, water, soil, etc.). This is accomplished via modelling of (thermodynamic) exchange processes such as partitioning, diffusion, sorption, advection, convection - represented as arrows in Fig. 10.18 - as well as biotic and abiotic degradation (e.g. biodegradation, hydrolysis or photolysis), or burial in sediments. Degradation is an important sink for most organic substances, but may also lead to toxic breakdown compounds. The rate by which the degradation occurs can be described by the half-life of the substance in the medium and it depends both on the properties of the substance and on environmental conditions such as temperature, insolation or presence of reaction partners (e.g. $\mathrm{OH}$ radicals for atmospheric degradation). The basic principle underlying a fate model is a mass balance for each compartment leading to a system of differential equations which is solved simultaneously, which can done for steady-state or dynamic conditions. A life cycle inventory typically reports emissions as masses emitted into an environmental compartment for a given functional unit, but the mathematical relationship between the steady-state solution for a continuous emission and the time-integrated solution for a mass of chemical released into the environment has been demonstrated (Heijungs 1995; Mackay and Seth 1999).

Figure 10.18 shows the overall nested structure of the USEtox model which is a widely used global scientific consensus model for characterisation modelling of human and ecotoxic impacts in LCA. Further details on fate modelling principles in the USEtox model can be found in Henderson et al. (2011) and Rosenbaum et al. (2008).

Exposure is the contact between a target and a pollutant over an exposure boundary for a specific duration and frequency. The exposure model accounts for the fact that not necessarily the total ('bulk') chemical concentration present in the environment is available for exposure of organisms. Several factors and processes such as sorption, dissolution, dissociation and speciation may influence (i.e. reduce) the amount of chemical available for ecosystem exposure. Such phenomena can be defined as bioavailability ("freely available to cross an organism's cellular membrane from the medium the organism inhabits at a given time"), and bioaccessibility ("what is actually bioavailable now plus what is potentially bioavailable").

The effect model characterises the fraction of species within an ecosystem that will be affected by a certain chemical exposure. Effects are described quantitatively by lab-test derived concentration-response curves relating the concentration of a chemical to the fraction of a test group that is affected (e.g. when using the EC50the Effect Concentration affecting 50\% of a group of individuals of the same test species compared to a control situation). Affected can mean various things, such as increased mortality, reduced mobility, reduced growth or reproduction rate, 


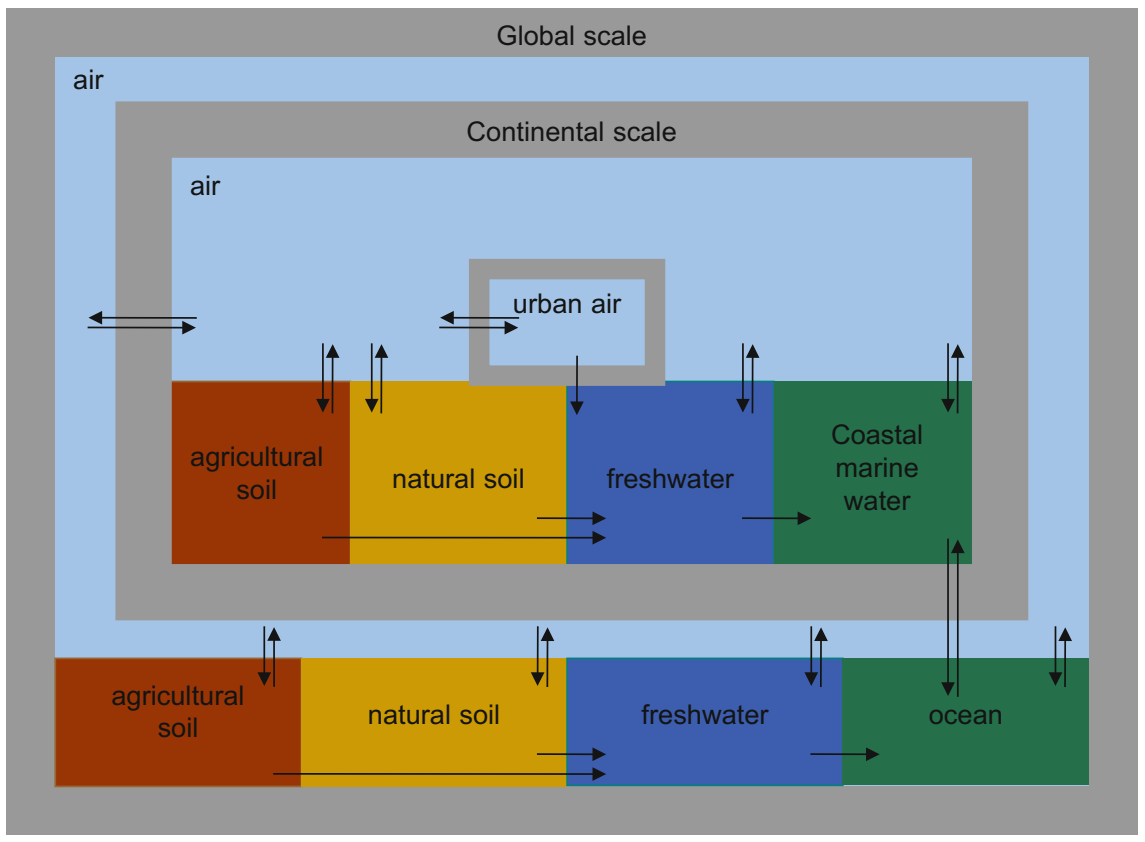

Fig. 10.18 The USEtox fate model [taken from Rosenbaum et al. (2008)]

mutations, behavioural changes, changes in biomass or photosynthesis, etc. These are the effects that may be observed during standardised laboratory-based ecotoxicity tests, and the results are specific for each combination of substance and species. Toxic effects are further distinguished into acute, sub-chronic and chronic toxicity (including further sub-groups like sub-acute, etc.). Acute toxicity describes an adverse effect after a short period of exposure, relative to the lifetime of the animal (e.g. $<7$ days for vertebrates, invertebrates or plants and $<3$ days for algae). Chronic toxicity is based on exposure over a prolonged period of time covering at least one life cycle or one sensitive period (e.g. $\geq 32$ days for vertebrates, $\geq 21$ days for invertebrates, $\geq 7$ days for plants and $\geq 3$ days for algae).

When relating to freshwater ecosystems, the question arises what exactly we mean by that. In LCIA, a freshwater ecosystem is typically seen as consisting of at least three trophic levels:

1. Primary producers, converting sunlight into biomass via photosynthesis (i.e. phytoplankton, algae)

2. Primary consumers, living off primary producers (i.e. zooplankton, invertebrates, planktivorous fish)

3. Secondary consumers at the upper end of the aquatic food chain (i.e. piscivorous fish) 
It should be noted that only impacts on cold-blooded species in freshwater ecosystems are currently considered. There is no minimum requirement established, which trophic levels should be covered by a characterisation factor for terrestrial or marine ecosystems and available methods usually extrapolate from freshwater data or use the relatively few data available directly for these ecosystems.

There is often a large variation of sensitivity to a given substance between different species in the freshwater ecosystem. This is described by a species-sensitivity-distribution (SSD) curve, which hence represents the sensitivity of the entire ecosystem to a substance-see Fig. 10.19.

The SSD is constructed using the respective geometric mean of all available and representative toxicity values for each species. This curve represents the range of sensitivity to exposure to a given substance among the different species in an ecosystem from the most sensitive to the most robust species. The ecotoxicity effect factor is then calculated using the HC50-Hazardous Concentration at which $50 \%$ of the species (in an aquatic ecosystem) are exposed to a concentration above their EC50, according to the SSD curve (see Fig. 10.19). The dimension of the effect factor is PAF-Potentially Affected Fraction of species, while the unit is typically $\mathrm{m}^{3} / \mathrm{kg}$.

The ecotoxicological effect factor of a chemical is calculated as:

$$
\mathrm{EF}_{\mathrm{eco}}=\frac{0.5}{\mathrm{HC} 50}
$$

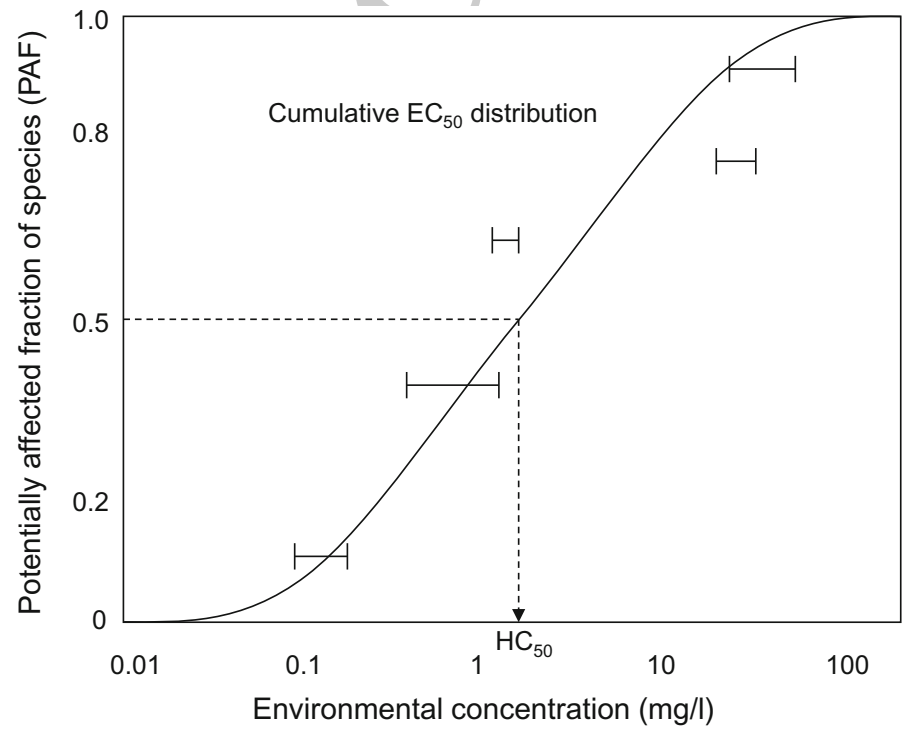

Fig. 10.19 Species-sensitivity distribution (SSD) curve representing the sensitivity of the ecosystem to a chemical substance 
The HC50 value can be determined from the SSD curve but is often, more conveniently, calculated as the geometric mean of the EC50 values per species s, respectively:

$$
\log \mathrm{HC} 50=\frac{1}{n_{\mathrm{s}}} \cdot \sum_{\mathrm{s}} \log \mathrm{EC} 50_{\mathrm{s}}
$$

where $n_{\mathrm{s}}$ is the number of species.

A damage model, incorporating the severity of the effect, goes even further along the cause-effect chain and quantifies how many species are disappearing (instead of 'just' affected) from a given ecosystem. Disappearance may be caused by mortality, reduced proliferation or migration, for example.

\subsubsection{Emissions and Main Sources}

Chemicals are a main pillar of our industrialised economy, they are used in virtually any product around the globe and therefore numerous, used in large quantities and emitted from nearly all processes that an LCI may contain. Ecotoxity is very different from any other (non-toxicity) impact category when it comes to the number of potentially relevant elementary flows. Whereas no other (non-toxicity) impact category - with the exception of photochemical ozone formation-exceeds 100 contributing elementary flows (characterisation factors), the toxicity categories are facing the challenge of having to characterise several tens of thousands of chemicals with huge differences in their abilities to cause toxic impacts. The CAS registry currently (end 2016) contains more than 124 million unique organic and inorganic substances (www.cas.org/about-cas/cas-fact-sheets) of which roughly 200,000 may play an industrial role as reflected by the ever increasing number of more than 123,000 substances registered in the European Classification and Labelling Inventory Database which contains REACH (Registration, Evaluation, Authorisation and Restriction of Chemical substances) registrations and CLP (Classification, Labelling and Packaging of substances and mixtures) notifications so far received by the European Chemicals Agency (ECHA: http://echa.europa.eu/ information-on-chemicals/cl-inventory-database). Current LCIA models cover around 3000 substances for aquatic ecotoxicity.

\subsubsection{Existing Characterisation Models}

Characterisation methods like EDIP account for fate and exposure relying on key properties of the chemical applied to empirical models. Mechanistic models and methodologies have been published accounting for fate, exposure and effects 
providing cardinal impact measures. Among these methods are IMPACT 2002 (used in IMPACT 2002+) and USES-LCA (used in CML and ReCiPe). All these methods adopt environmental multimedia, multipathway models employing mechanistic cause-effect chains to account for the environmental fate, exposure and effects processes. However, they do not necessarily agree on how these processes are to be modelled, leading to variations in results of LCA studies related to the choice of LCIA method. Based on an extensive comparison of these models followed by a scientific consensus process, the scientific consensus model USEtox (UNEP/SETAC toxicity consensus model) was developed with the intention to solve this situation by representing a scientifically agreed consensus approach to the characterisation of human toxicity and freshwater ecotoxicity (Hauschild et al. 2008; Rosenbaum et al. 2008; Henderson et al. 2011). It has been recommended and used by central international organisations like the United Nations Environment Program UNEP, Society of Environmental Toxicology and Chemistry SETAC, the European Union and USE-EPA to characterise human and ecotoxicity in LCIA.

Among the existing characterisation models on midpoint level, three main groups can be distinguished: (1) mechanistic, multimedia fate, exposure and effect models, (2) key property-based partial fate models and (3) non-fate models (EC-JRC 2011). According to ISO 14044 (2006b) "Characterisation models reflect the environmental mechanism by describing the relationship between the LCI results, category indicators and, in some cases, category endpoints. [...] The environmental mechanism is the total of environmental processes related to the characterisation of the impacts." Therefore, ecotoxicity characterisation models falling into categories (2) and (3), do not completely fulfil this criterion. Caution is advised regarding their use and most importantly the interpretation of their results, which should not be employed without prior in-depth study of their respective documentation. Having said that, depending on the goal and scope of the LCA, they may still be an adequate choice in some applications, and indeed may agree quite well with the more sophisticated multimedia-based models.

Ecotoxicity endpoint modelling is still in an early state and much research needs to be performed before maturity is reached. The authors of the ILCD LCIA handbook concluded that "For all the three evaluated endpoint methods (EPS2000, ReCiPe, IMPACT 2002+), there is little or no compliance with the scientific and stakeholder acceptance criteria, as the overall concept of the endpoint effect factors is hardly validated and the endpoint part of the methods is not endorsed by an authoritative body. [...] No method is recommended for the endpoint assessment of ecotoxicity, as no method is mature enough." (EC-JRC 2011).

When interpreting the results of existing methods, it is important to keep in mind that many aspects are not or only very insufficiently covered. This includes elements like terrestrial and marine ecotoxicity as well as toxicity of pesticides to pollinators.

For further details see Chap. 40 and Hauschild and Huijbregts (2015). 


\subsection{Human Toxicity}

As explained in Sect. 10.11, both toxicity impact categories have a number of things in common, like main emissions and sources, modelling principles, model structure and even some of the models used in the characterisation are identical between the human toxicity and ecotoxicity impact categories. Notably the fate model used is the same in LCIA methods using mechanistic characterisation modelling, which is the majority of existing methods. Therefore, only those parts that are specific for human toxicity and different from ecotoxicity will be discussed here. It is recommended to first read Sect. 10.11 in order to understand the main underlying principles not repeated hereafter.

\subsubsection{Problem}

Human toxicity in LCA is based on essentially the same driving factors as ecotoxicity: (1) emitted quantity (determined in the LCI), (2) mobility, (3) persistence, (4) exposure patterns and (5) human toxicity, with the latter four considered by the characterisation factor. The respective mechanisms and parameters are certainly different and specific for human toxicity, notably for the exposure modelling, where many factors capturing human behaviour, such as dietary habits, that influence human exposure pattern.

Chemical exposure of humans can result from emissions into the environment which will affect the whole population, but also from the many chemical ingredients in products released during their production, use, or end-of-life treatment and thus affecting workers or consumers. Chemical emissions are responsible for, or contribute to, many health impacts such as a wide range of non-cancer diseases as well as increased cancer risks for those chemicals that are carcinogenic.

\subsubsection{Environmental Mechanism}

Modelling the toxicological effects on human health of a chemical emitted into the environment, whether released on purpose (e.g. pesticides applied in agriculture), as a by-product from industrial processes, or by accident, implies a cause-effect chain, linking emissions and impacts through four consecutive steps as depicted in Fig. 10.20.

The cause-effect chain links the emission to the resulting mass in the environmental compartments (fate model) and on to the intake of the substance by the overall population via food and inhalation exposure pathways (human exposure model), and to the resulting number of cases of various human health risks by comparison of exposure with the known dose-response relationship for the 


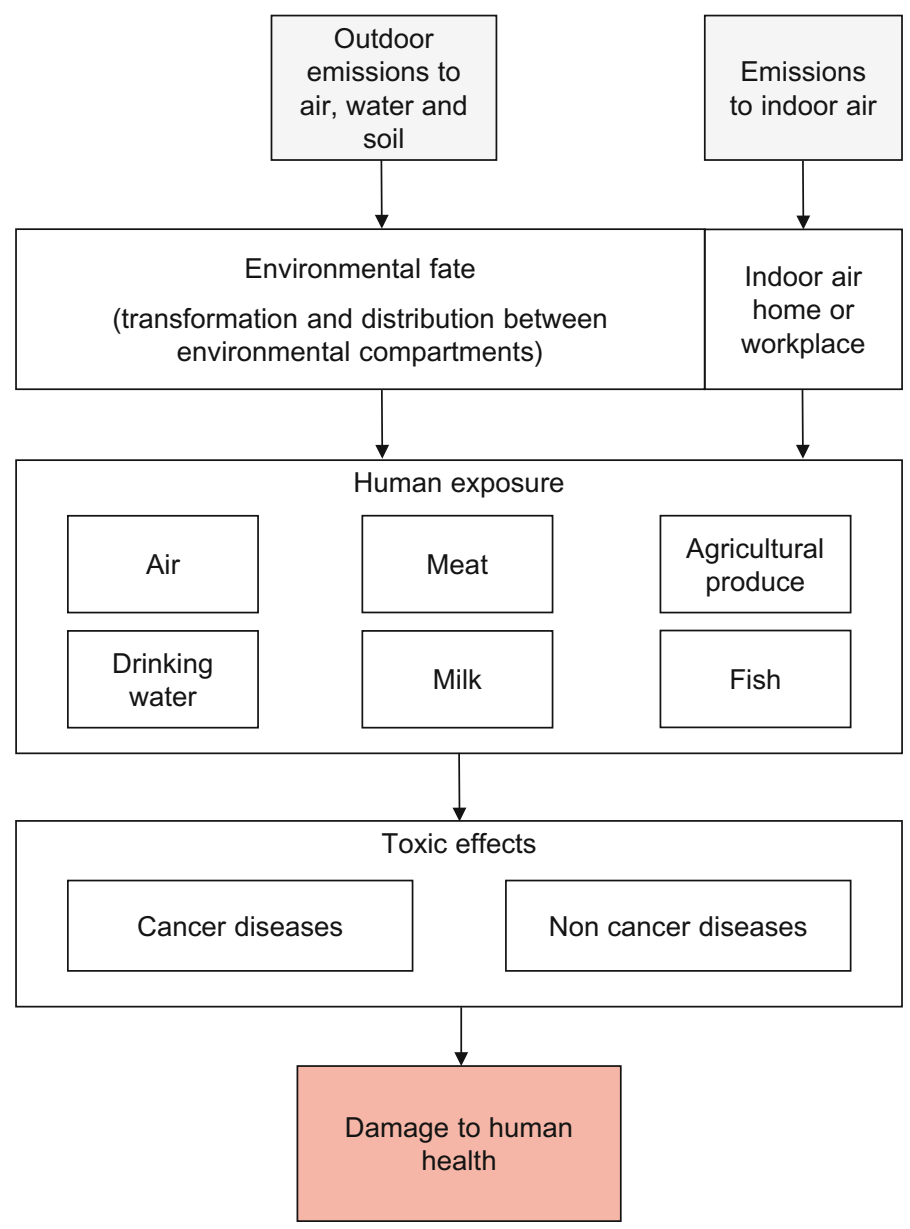

Fig. 10.20 General scheme of the impact pathway for human toxicity [adapted from EC-JRC (2011)]

chemical (toxic effect model) and finally their damage to the health of the overall population. In the characterisation modelling, the links of this cause-effect chain are expressed, similarly to Eq. 10.6, as factors corresponding to the successive steps of fate, exposure, effects and damage:

$$
\mathrm{CF}_{\mathrm{hh}}=\mathrm{FF} \times \mathrm{XF}_{\mathrm{hh}} \times \mathrm{EF}_{\mathrm{hh}} \times \mathrm{SF}_{\mathrm{hh}}
$$

where $\mathrm{CF}_{\mathrm{hh}}$ is the human health characterisation factor, FF the fate factor, $\mathrm{XF}_{\mathrm{hh}}$ the human exposure factor, $\mathrm{EF}_{\mathrm{hh}}$ the human toxicity effect factor (midpoint effects) and $\mathrm{SF}_{\mathrm{hh}}$ the human health severity factor (endpoint effects). Some LCIA methods also directly combine $\mathrm{EF}_{\mathrm{hh}}$ and $\mathrm{SF}_{\mathrm{hh}}$ into a single damage factor, directly calculating an 
endpoint characterisation factor. For midpoint characterisation, $\mathrm{SF}_{\mathrm{hh}}$ is simply omitted and $\mathrm{CF}_{\mathrm{hh}}$ is then the midpoint human toxicity (i.e. not human health) characterisation factor.

The midpoint human toxicity characterisation factor [number of cases $/ \mathrm{kg}_{\mathrm{emitted}}$ ] expresses the toxic impact on the global human population per mass unit emitted into the environment and can be interpreted as the increase in population risk of disease cases due to an emission into a specific environmental compartment. The endpoint human health characterisation factor [DALY $/ \mathrm{kg}_{\text {emitted] }}$ quantifies the impact on human health in the global population in Disability-Adjusted Life Years (DALY) per mass unit emitted into the environment. DALY is a statistical measure of population life years lost or affected by disease (or other influences) and is used among other by the World Health Organisation.

The fate model is, without exception, the same as for ecotoxicity. Logically, the environment in which a chemical is transported, distributed and transformed is the same, no matter who will be affected. Therefore, for the sake of consistency, all LCIA methods that cover human toxicity are using the same fate model as for ecotoxicity, but of course different exposure and effect models, as this will be specific for the targeted organism (human or animal). The fate model is therefore described in Sect. 10.11.

The exposure model relates the amount of chemical in a given environmental compartment to the chemical intake by humans (exposure rates). It can be differentiated into direct intake (e.g. by breathing air and drinking water), indirect intake through bioconcentration processes in animal tissues (e.g. meat, milk and fish) and intake by dermal contact. An exposure pathway is defined as the course a chemical takes from the environment to the exposed population, for example through air, meat, milk, fish, water or vegetables. Exposure pathways can be further aggregated into exposure routes, such as inhalation of air, ingestion of food including drinking water and other matter such as soil particles and dermal exposure. The human exposure model is designed for assessing human exposure to toxic chemical emissions applying realistic exposure assumptions and being adapted to take spatial variability into account. In LCIA human exposure is always assessed at the population level.

The intake Fraction $\mathrm{iF}$ is calculated as the product of fate and exposure factor $\left(\mathrm{iF}=\mathrm{FF} * \mathrm{XF}_{\mathrm{hh}}\left[\mathrm{kg}_{\text {intake }} / \mathrm{kg}_{\text {emitted }}\right]\right)$ and it can be interpreted as the fraction of an emission that is taken in by the overall population through all exposure routes, i.e. as a result of food contamination, inhalation and dermal exposure. A high value, such as $\mathrm{iF}=0.001$ for dioxins, reflects that humans will take in 1 part out of 1000 of the mass of a chemical released. Dioxins are very efficient in exposing humans as reflected by the high intake fraction. For other chemicals, values typically lie in the range of $10^{-10}$ to $10^{-5}$.

The effect model relates the quantity of a chemical taken in by the population via a given exposure route (inhalation and ingestion, respectively, dermal uptake is normally not modelled in LCIA) to the toxic effects of the chemical once it has entered the human organism and can be interpreted as the increase in the number of cases of a given human health effect (e.g. cancer or non-cancer diseases) in the exposed population per unit mass taken in. The two general effect classes, cancer 
and non-cancer, each cover a multitude of different diseases, so this is a simplification reflecting the fact that it is very difficult to predict the many underlying human toxicity endpoints from the animal dose-response curves from laboratory experiments with test animals which are normally the basis of the effect factor.

The severity factor represents adversely affected life years per disease case (DALY/case), distinguishing between differences in the severity of disabilities caused by diseases in terms of affected life years, e.g. discriminating between a lethal cancer and a reversible skin irritation. It is quantified by the statistically determined, population-based years of life lost (YLL) and years of life disabled (YLD) due to a disease.

\subsubsection{Emissions and Main Sources}

The relevant emissions and main sources are identical to those of the ecotoxicity impact category and discussed in Sect. 10.11.

\subsubsection{Existing Characterisation Models}

Again here, Sect. 10.11 contains a discussion on existing characterisation models, which largely applies also to the human toxicity impact category.

In USEtox, the units of the two human toxicity midpoint indicators for non-cancer and cancer are Comparative Toxic Unit for humans $\mathrm{CTU}_{\mathrm{h}}$ in [disease cases $/ \mathrm{kg}_{\text {emitted }}$. They can be added up to a single human health indicator, but then the interpretation needs to consider that this intrinsically assumes equal weighting between cancer and non-cancer effects (which includes equal weighting between e.g. a reversible skin rash and non-reversible death). Human health endpoint indicators in USEtox are given in the Comparative Damage Unit for human health $\mathrm{CDU}_{\mathrm{h}}$ in [DALY $\left./ \mathrm{kg}_{\mathrm{emitted}}\right]$. In accordance with the purpose of endpoint modelling, this indicator better represents the distinction of the severity of different effects.

When interpreting human toxicity indicators from existing methods, it is important to be aware that these only provide indicators for global population exposure to outdoor and indoor emissions, while human toxicity for occupational exposure of workers or direct exposure related to product use for consumers are not yet covered by USEtox and the other characterisation models, despite their very high relevance. Products of special interest in this context are cosmetics, plant protection products, textiles, pharmaceuticals and many others, that may in particular contain substances having toxic properties and have the potential to cause mutagenic, neurotoxic or endocrine disrupting effects. This is the subject of ongoing research and will be included in LCIA methods once the models are mature and operational.

For further details see Chap. 40 and Hauschild and Huijbregts (2015). 


\subsection{Particulate Matter Formation}

In existing LCIA methods, health impacts from exposure to particulate matter as impact category is referred to by different terms (e.g. "particulate matter/respiratory inorganics' in ILCD, 'respiratory effects' in IMPACT 2002+, 'human health criteria pollutants' in TRACI, or 'particulate matter formation' in ReCiPe). Although causing mainly toxicity-related health effects, exposure to PM is considered a separate impact category in most LCIA methods. This is mainly due to a number of important differences between the characterisation of PM formation and that of human toxicity. These differences include the complex atmospheric chemistry involved in the formation of secondary PM from different precursor substances which requires a different fate model. Furthermore, different emission heights are important to consider, global monitoring data for PM air concentrations are used, and the effect assessment is based on exposure-response functions mostly derived from epidemiological evidence, which is not possible for most toxic chemicals due to missing emission locations and exposure- or dose-response information.

\subsubsection{Problem}

A large number of studies including the global burden of disease (GBD) study series consider particulate matter (PM) to be the leading environmental stressor contributing to global human disease burden (i.e. all diseases around the world) via occupational and household indoor exposure as well as urban and rural outdoor (ambient) exposures. In 2013, ambient PM pollution accounted for 2.9 million deaths and 70 million DALY, and household PM pollution from solid fuels accounted for 2.9 million deaths and 81 million DALY (Forouzanfar et al. 2015). With that, ambient and household PM pollution combined contributed in 2013 with $71 \%$ to premature deaths attributable to all environmental risk factors and with $19 \%$ to premature death attributable to all risk factors (i.e. including behavioural etc.). This means that exposure to PM accounts on average for 1 out of 5 premature deaths worldwide. Thereby, exposure to PM is associated in epidemiological and toxicological studies with various adverse health effects and reduction in life expectancy including chronic and acute respiratory and cardiovascular diseases, chronic and acute mortality, lung cancer, diabetes and adverse birth outcomes (Fantke et al. 2015).

PM can be distinguished according to formation type (primary and secondary) and accórding to aerodynamic diameter (respirable, coarse, fine and ultrafine). Primary PM refers to particles that are directly emitted, e.g. from road transport, power plants or farming activities. Secondary PM refers to organic and inorganic particles formed through reactions of precursor substances including nitrogen oxides $\left(\mathrm{NO}_{x}\right)$, sulphur oxides $\left(\mathrm{SO}_{x}\right)$, ammonia $\left(\mathrm{NH}_{3}\right)$, semivolatile and volatile organic compounds (VOC). Secondary particles include sulphate, nitrate and 
organic carbonaceous materials and can make up to $50 \%$ of ambient PM concentrations. Respirable particles $\left(\mathrm{PM}_{10}\right)$ have an aerodynamic diameter less than $10 \mu \mathrm{m}$, coarse particles $\left(\mathrm{PM}_{10-2.5}\right)$ between 2.5 and $10 \mu \mathrm{m}$, fine particles $\left(\mathrm{PM}_{2.5}\right)$ less than $2.5 \mu \mathrm{m}$, and ultrafine particles (UFP) less than $100 \mathrm{~nm}$ (WHO 2006). $\mathrm{PM}_{2.5}$ is often referred to as the indicator that best describes the component of PM responsible for adverse human health effects (Lim et al. 2012; Brauer et al. 2016).

\subsubsection{Environmental Mechanism}

Characterising health impacts from exposure to PM associated with emissions of primary PM or secondary PM precursor substances builds on the general LCIA framework for characterising emissions of air pollutants (see Fig. 10.2). The impact pathway for health impacts from PM emissions is illustrated in Fig. 10.21 and starts from primary PM emissions or secondary PM precursor substances emitted into air.

As for the toxicity impact categories, combining all factors from emission to health impacts or damages yields the characterisation factor for particulate matter formation $(\mathrm{CF})$ with units [disease cases $/ \mathrm{kg}_{\text {emitted }}$ ] at midpoint level (i.e. excluding $\mathrm{SF})$ and $\left[\mathrm{DALY} / \mathrm{kg}_{\mathrm{emitted}}\right]$ at endpoint level:

Impact Pathway

Mass emitted to air

[kg PM or precursor emitted]

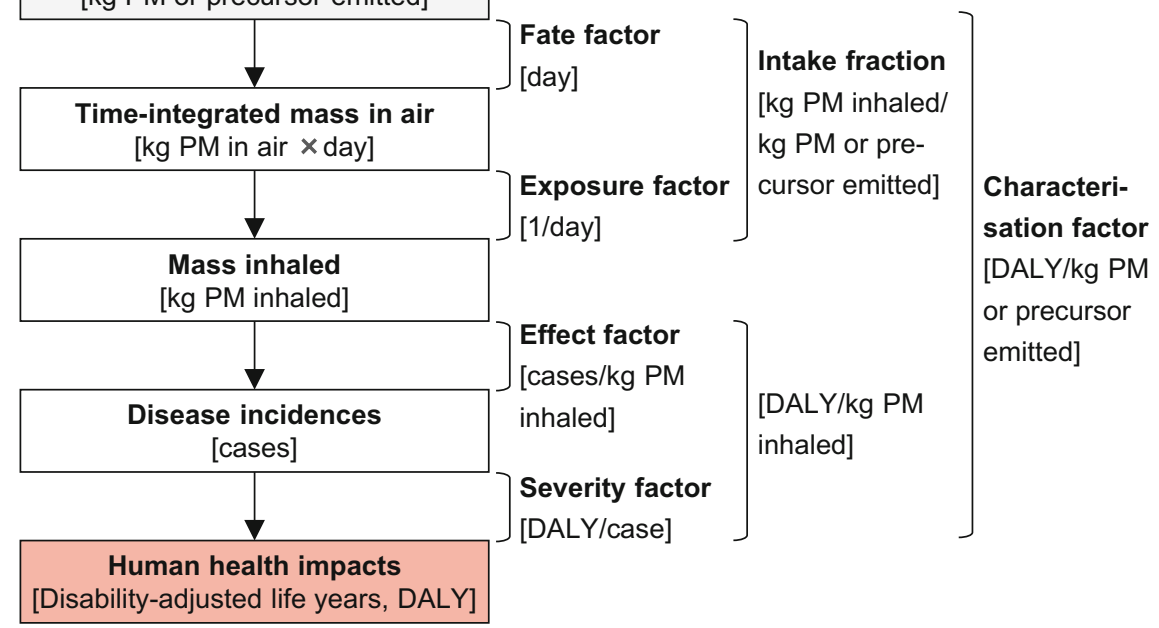

Fig. 10.21 Schematic impact pathway and related output metrics for characterising health impacts from particulate matter (PM) exposure in life cycle impact assessment [adapted from Fantke et al. (2015)] 


$$
\mathrm{CF}=\mathrm{FF} \times \mathrm{XF} \times \mathrm{EF} \times \mathrm{SF}
$$

Emissions are expressed as mass of PM or precursor substance released into air. From there, the impact pathway follows different distribution processes within and between air compartments and/or regions (indoor, outdoor, urban, rural, etc.) yielding a time-integrated mass of PM in the different air compartments and/or regions. Relating the time-integrated PM mass in air to the mass of PM or precursor substance emitted yields the fate factor (FF) with unit $\mathrm{kg}$ in air integrated over one day per $\mathrm{kg}$ emitted. A certain fraction of PM mass in air is subsequently inhaled by an exposed human population. This fraction is expressed by the exposure factor (XF) describing the rate at which PM is inhaled with unit $\mathrm{kg} \mathrm{PM}$ inhaled per $\mathrm{kg}$ PM in air integrated over one day. Multiplying FF and XF yields the cumulative PM mass inhaled by an exposed population per kg PM or precursor emitted expressed as human intake fraction (iF). Inhaling PM mass may then lead to a cumulative population risk referred to as expected disease incidences in the exposed human population and typically assessed based on PM air concentration. Relating PM concentration in air to cumulative population risk yields the exposure-response or effect factor (EF) with unit disease cases (e.g. death for mortality effects) per kg PM inhaled. Finally, disease incidences are translated into human health damages by accounting for the disease severity expressed as disability-adjusted life years (DALY) that include mortality and morbidity effects. Linking health damages to disease incidences yields the severity (or damage) factor (SF) with unit DALY per disease case.

For characterising health impacts from emissions of PM or precursor substances, several aspects influence emission, fate, intake and health effects. Regardless the modelling setup (spatial vs. archetypal; including or disregarding indoor sources and/or secondary PM formation, etc.), main influential aspects are spatiotemporally variable population density and activity patterns, background PM concentration in air, background disease rate and background severity, emission location (e.g. indoor vs. outdoor or urban vs. rural) and emission height, as well as potential nonlinearity in the disease-specific exposure-response relationship. The effect of using a non-linear exposure-response curve in the calculation of CFs following the marginal and average approach is illustrated in Fig. 10.22 for two distinct background concentration scenarios, where the difference between marginal and average approach is increasing with increasing background concentration for an exposure-response curve of supralinear shape.

\subsubsection{Emissions and Main Sources}

Substances considered in the different LCIA methods to contribute to health impacts from $\mathrm{PM}$ are typically one or more $\mathrm{PM}$ fractions $\left(\mathrm{PM}_{10}, \mathrm{PM}_{10-2.5}, \mathrm{PM}_{2.5}\right)$ 


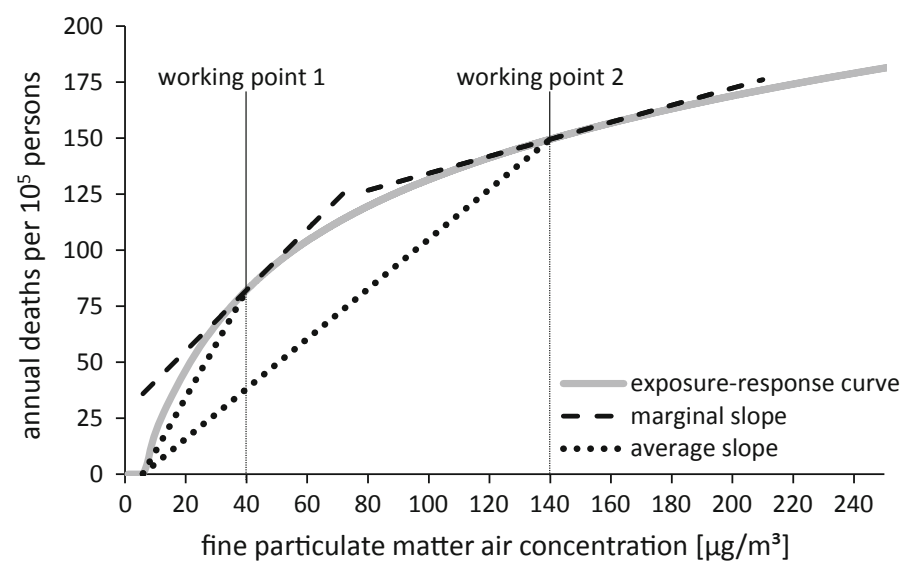

Fig. 10.22 Illustration of using a non-linear exposure-response curve for health effects from fine particulate matter exposure with dashed and dotted lines as approaches for calculating marginal and average (between working point and theoretical minimum-risk concentration) characterisation factors, respectively, at different background concentrations in air as working points. Exposure-response curve based on Apte et al. (2015)

and $\mathrm{PM}$ precursor substances (mostly $\mathrm{NO}_{x}, \mathrm{SO}_{2}$ and $\mathrm{NH}_{3}$ ) and in some cases also carbon monoxide (e.g. IMPACT 2002+) or non-methane volatile organic compounds (e.g. ReCiPe). Relevant emission sources of PM (and/or precursors) are for example road traffic, stationary emissions from coal/gas-fired power plants or indoor emissions from solid fuels combustion. Several emission sources are ground-level sources (e.g. road traffic and household combustion), while others are considered to occur at higher stack levels (typically stationary emission sources, e.g. power plants).

\subsubsection{Existing Characterisation Models}

In LCIA, archetypal impact assessment scenarios (e.g. urban, rural, etc.) are often used instead of spatialized or site-specific scenarios, especially when emission locations are unknown or fate, exposure and/or effect data do not allow for spatial differentiation. Such archetypal approach and related intake fractions were proposed by Humbert et al. (2011) with population density (urban, rural and remote) and emission height (ground-level, low-stack and high-stack emissions) as main determinants of PM and precursor impacts. The UNEP/SETAC Life Cycle Initiative established a task force to build a framework for consistently quantifying health effects from PM exposure and for recommending PM characterisation factors for application in LCIA with fine particulate matter $\left(\mathrm{PM}_{2.5}\right)$ as representative indicator. First recommendations from this task force focus on the integration of 
indoor and outdoor environments, the archetypal approach capturing best the dominating differences between urban and rural areas and a number of other improvements (Fantke et al. 2015).

Most LCIA characterisation methods addressing particulate matter formation follow the framework described in this section. There are some methods, however, that characterise impacts from particles as part of the 'human toxicity' impact category (e.g. CML 2002 and EDIP 2003), while most methods (including all methods developed after 2010) characterise human toxicity impacts from chemicals and impacts from particles as separate impact categories, mainly due to the differences in available data that allow using more refined models and less generic assumptions for the impact assessment of particle emissions.

The most recent characterisation models - all damage-oriented - include work by van Zelm et al. (2008) providing characterisation factors for primary and secondary $\mathrm{PM}_{10}$ for Europe based on a source receptor model, work by Gronlund et al. (2015) giving archetypal characterisation factors for primary $\mathrm{PM}_{2.5}$ and secondary $\mathrm{PM}_{2.5}$ precursors based on US data and work by van Zelm et al. (2016) proposing averaged primary and secondary $\mathrm{PM}_{2.5}$ characterisation factors for 56 world regions based on a global atmospheric transport model. However, none of the currently available approaches includes indoor sources, is able to distinguish emission situations at the city level or considers the non-linear nature of available exposure-response curves, which is why further research is needed for this impact category. For further details see Chap, 40 and Hauschild and Huijbregts (2015).

\subsection{Land Use}

\subsubsection{Problem}

Land use refers to anthropogenic activities in a given soil area. Examples of land use are agricultural and forestry production, urban settlement and mineral extraction. The land use type in a specific area can be identified by the physical coverage of its surface, for example tomato crop grows in open-field orchards or under greenhouses, artificial surfaces with infrastructure are the expression of human settlements and open-pits are a sign of ore extraction. There is thus a direct link between land use and land cover, which is used to analyse land use dynamics and landscape change patterns.

Soil is a finite resource, which contributes to the environmental consequences of its use. Soil loss actually occurs quantitatively with the average soil formation rate being extremely low compared to the soil depletion rate. It also affects qualitative soil attributes, because degrading takes place via unsustainable management practices for the highest quality soils, which are those able to fulfil a greater diversity of purposes. As soil or land surface available at a given time is limited, land-use competition between resource users for occupying the same space often 
arises. This drives continuous changes in land uses. Croplands, pastures, urban areas and other land-use intensive, human activities have expanded worldwide in the last decades at the expense of natural areas to satisfy our growing society's needs for food, fibre, living space and transport infrastructure. Such changes transform the planet's land surface and lead to large and often irreversible impacts on ecosystems and human quality of life (EEA 2010). For example, forest clearing contributes to climate change with the release of carbon from the soil to the atmosphere. The loss, fragmentation and modification of habitats lead to biodiversity decline. Land use change alters the hydrological cycle by river diversion and by modifying the portion of precipitation into runoff, infiltration and evapotranspiration flows (Foley et al. 2005). After soil surface conversion, inappropriate management practices on human-dominated lands can also trigger a manifold of environmental effects on soil physical properties. In agricultural lands, mechanised farming can induce soil compaction, which affects aquifer recharge and the natural capacity of the soil to remove pollutants. Erosion is also a spread environmental concern of intensive agricultural practices. In urban and industrial areas, soil has been replaced by concrete surfaces and all its functions annulled.

The Millennium Ecosystem Assessment/(2005) provides a comprehensive description of how human land-use activities affect biodiversity and the delivery of ecological functions. Some ecological effects of land use are:

- Biodiversity decrease at the ecosystem, species and genetic levels

- Impacts on local and regional climate regulation due to changes in land cover and albedo, e.g. tropical deforestation and desertification may locally reduce precipitation

- Regional decline in food production per capita due to soil erosion and desertification, especially in dry lands

- Rise in flood and drought risks through loss of wetlands, forests and mangroves

- Change in the water cycle by river diversion and by greater appropriation of freshwater from rivers, lakes and aquifers to be used for irrigation of areas converted to agriculture

To sum up, land-use activities (including land conversion and land use itself) cause noticeable damages on biodiversity and on the performance of soil to provide ecological functions as illustrated in Fig. 10.23. These ecological functions upon which human well-being depends are also referred to as ecosystem services (Millenium Ecosystem Assessment 2005), and together with biodiversity loss are the focus of the LCIA land-use impact category.

\subsubsection{Environmental Mechanism}

The LCIA land-use impact category covers a range of consequences of human land use, being a receptacle (or 'bulk') category for many impact indicators. It does not 
Fig. 10.23 The land use impact category focuses on damage to biodiversitywhich represents the foundation of ecosystems - as well as on the provision of ecosystem services, due to land conversion and land use [adapted from Millenium Ecosystem Assessment (2005)]

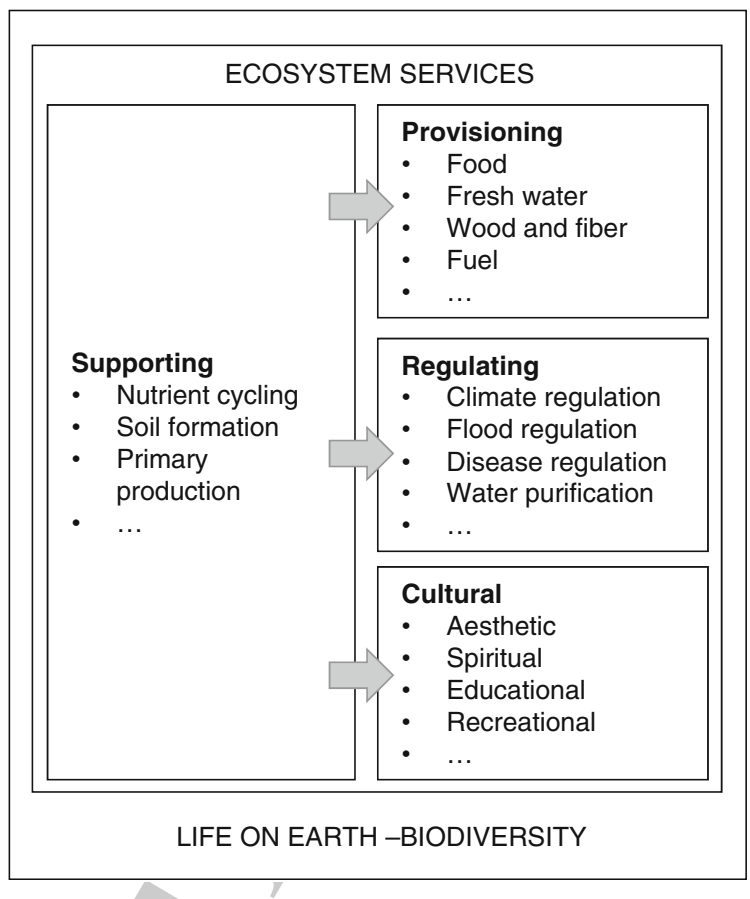

assess nutrients, pesticides and any other types of emission to the ecosphere which are characterised by the corresponding emission-based impact category (e.g. eutrophication for emission of nutrients, ecotoxicity for emission of pesticides). Their inclusion in the land-use category would lead to double counting of the same impact.

The general land-use environmental mechanism follows the model of Fig. 10.24. It shows the cause-effect chain from the elementary flow (i.e. land transformation or land occupation) to the endpoint damages on human health and ecosystems as well as available soil resources. Land transformation refers to the conversion from one state to another (also known as land use change, LUC) and land occupation to the use of a certain area for a particular purpose (also known as land use, LU). The figure should be read as follows, giving an example of the depicted impact pathways: land occupation leads to physical changes to soil, which leads to an altered soil function and affects habitats and net primary production which eventually leads to damage on ecosystem quality. The picture provides a good display of the complexity involved in land-use modelling. For some of the presented impacts, such as warming effect due to albedo change or landscape impairment, characterisation models have yet to be developed.

The same type of human activity may cause different land-use related impacts depending on the region of the world where the activity takes place. This variation is due to the strong influence of climate, soil quality, topography and ecological quality on the magnitude of the impact. For example, deforestation of a forest area 
Life cycle inventory (LCl)

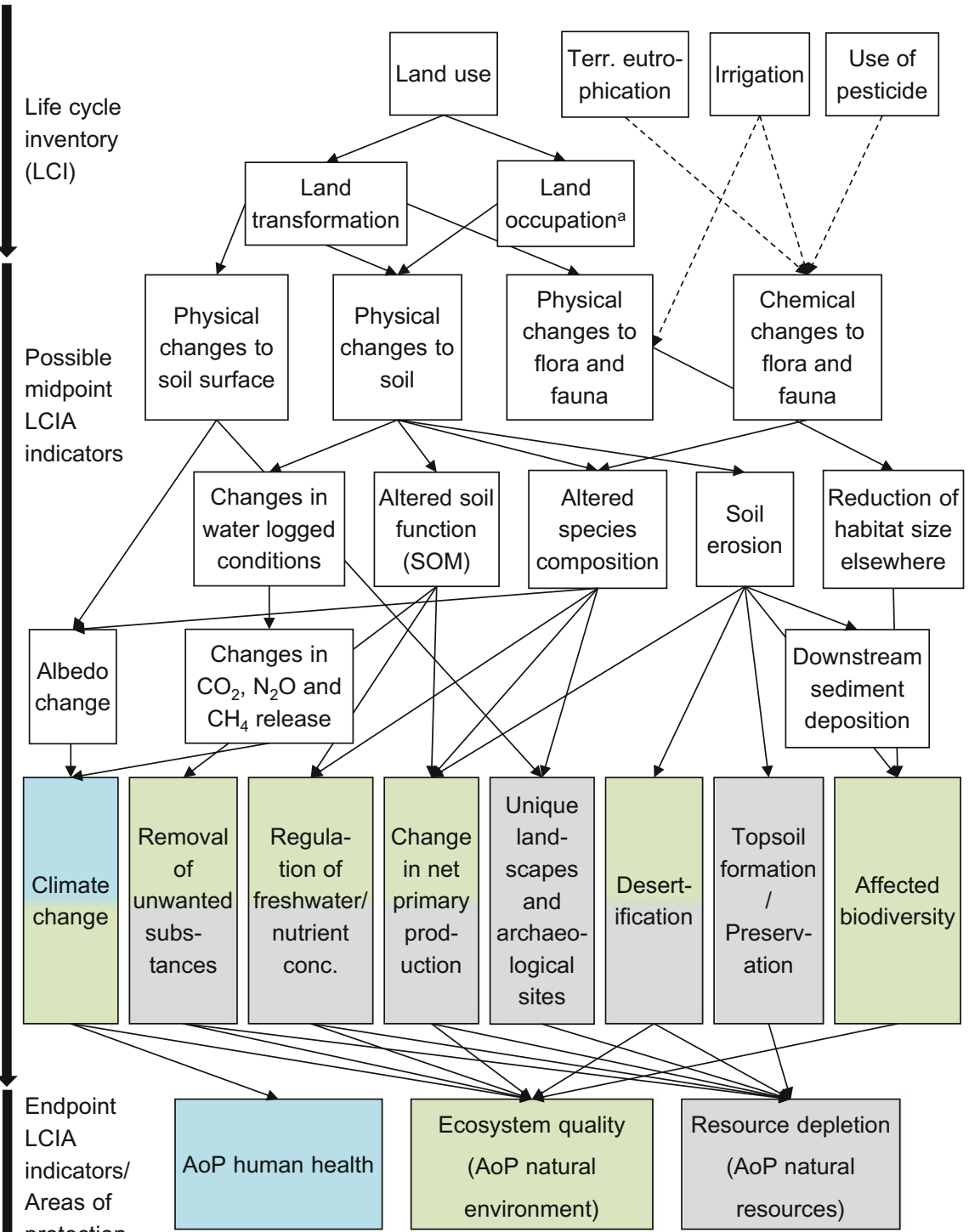

protection

Fig. 10.24 Impact pathway for land use impacts; dashed arrows indicate impacts covered by emission-related impact categories and by water use in the case of irrigation [adapted from EC-JRC (2011)]. ${ }^{a}$ Land occupation will not cause changes but will contribute to prolong the changed conditions

for use in agriculture in the Brazilian Amazon has a greater impact in terms of number of species affected than forest clearing in an ecologically poorer European region. Because land use impacts depend on-site-specific conditions, land use is 
considered a local impact category in LCA, in opposition to other impact categories of global geographic scope such as climate change, whose environmental effects (in terms of radiative forcing) are independent of the location of the emission.

As a consequence of the above explanation, methods that focus on land-use impacts should include geospatial data both in the LCI and the LCIA phases. The inventory must contain information on the geographic location of the human intervention, with a level of detail that may vary from the exact coordinates to coarser scales (e.g. biome, country, continent), depending on the goal and scope of the study and if the inventory refers to the foreground or to the background system (see Chap. 9). In the LCIA, characterisation factors for a given impact indicator must capture the sensitivity of the habitat to the impact modelled. For example, characterisation factors for soil erosion may include information on the soil depth in the specific location of the activity under evaluation, as the impact of soil loss will depend on the soil stock size, i.e. thinner soils are more vulnerable than thicker soils (Núñez et al. 2013). Every geographic unit of regionalised impact assessment methods has its own characterisation factor. Within the boundary of such a unit, it is assumed that an activity triggers the same impacts on land.

\subsubsection{Existing Characterisation Models}

Characterisation of land use in LCA has been extensively discussed over the last decades but is far from being settled, because the first operational methods have only been available since 2010 . Until then, land use was only an inventory flowcounted in units of surface occupied and time of occupation $\left(\mathrm{m}^{2}\right.$ and years) and surface transformed $\left(\mathrm{m}^{2}\right)$, without any associated impact. The main reason for this "late development" is that land-use related impacts rely on spatial and temporal conditions where the evaluated activity takes place, whereas traditional LCA is site-generic. During the last few years, the release of geographical information system (GIS) software and data sets have brought new opportunities in LCA to model land-use impacts and in general, any other spatially dependent impact category.

Today, there are LCIA methods to evaluate impacts on biodiversity and impacts on several ecosystem services. From the long list of services provided by terrestrial ecosystems (24 acknowledged in the Millennium Ecosystem Assessment international work programme (2005), LCA focuses on those which are recognised as being more environmentally relevant (i.e. educational and spiritual values are excluded). A non-exhaustive list of methods is provided below. For completeness, see Milâ i Canals and de Baan (2015):

- Impacts on biodiversity: Biodiversity should be preserved because of its intrinsic/value. The most commonly applied indicator is based on species richness, given the availability of data (Koellner and Scholz 2007, 2008; de Baan et al. 2013a, b). Damage on biodiversity is commonly expressed in 
quantity of species biodiversity loss, either in relative terms (potentially disappeared fraction of species times surface, PDF. $\mathrm{m}^{2}$ ) or in absolute species loss. Existing indicators for biodiversity are at the endpoint level (in Fig. 10.24, Ecosystem quality-AoP natural environment box in the lower row). The UNEP-SETAC Life Cycle Initiative project on global guidance for LCIA indicators and methods provisionally recommended characterisation factors from Chaudhary et al. (2015) representing global potential species loss from land use to assess impacts on biodiversity due to land use and land-use change as hotspot analysis in LCA only (not for comparative assertions nor eco-labelling). Further testing of the CFs as well as the development of CFs for further land-use types are required to provide full recommendation.

- Impacts on ecosystem services: Includes a range of indicators for life support functions that ecosystems provide. Ecosystem services are hardly covered in LCIA and proposals are still incipient. All available methods are on the midpoint level (in Fig. 10.24, boxes between the LCI and the endpoint), which means that comparison or aggregation with damages on biodiversity is not possible so far. The recent draft review of land-use characterisation models for use in Product and Organisation Environmental Footprint (PEF/OEF) provisionally (i.e. "apply with caution") recommended characterisation factors from LANCA (Bos et al. 2016) to assess impacts on ecosystem services (EC-JRC 2016). Currently, there are LCA methods for the following ecosystem services:

- Biotic production potential: capacity of ecosystems to produce and sustain biomass on the long term. Available indicators are based on the soil organic matter (or carbon) content (Brandão and Milà i Canals 2013), the biotic production (Bos et al. 2016) and the human appropriation of the biotic production (Alvarenga et al. 2015)

- Carbon sequestration potential: capacity of ecosystems to regulate climate by carbon uptake from the air. The size of the climatic impact is determined by the amount of $\mathrm{CO}_{2}$ transfers between vegetation/soil and the atmosphere in the course of terrestrial release and re-storage of carbon (Müller-Wenk and Brandão 2010)

- Freshwater regulation potential: capacity of ecosystems to regulate peak flow and base flow of surface water. Available indicators refer to the way a land-use system affects average water availability, flood and drought risks, based on the partition of precipitation between evapotranspiration, groundwater infiltration and surface runoff (Saad et al. 2013; Bos et al. 2016)

- Water purification potential: mechanical, physical and chemical capacity of ecosystems to absorb, bind or remove pollutants from water. Site-specific soil properties such as texture, porosity and cation exchange capacity are used as the basis for the assessment (Saad et al. 2013)

- Erosion regulation potential: capacity of ecosystems to stabilise soils and to prevent sediment accumulation downstream. The soil performance is determined by the amount of soil loss (Saad et al. 2013; Bos et al. 2016) and how this soil loss reduces the on-site soil reserves and the biotic production (Núñez et al. 2013) 
- Desertification regulation potential: capacity of dry lands to resist irreversible degradation on the human time-frame. A multi-indicator system of four variables, namely climate aridity, soil erosion, aquifer exploitation and fire risk, determines the desertification ecosystem vulnerability (Núñez et al. 2010)

The land-use impact category is likely the LCA category most affected by potential problems of double counting. This is because methods for emissions and methods for land use have been developed under two different, incompatible approaches. Emission models are bottom-up: the starting point is the elementary flowin the LCI and the impact model describes stepwise all the mechanisms that link the cause (the LCI) to the consequence (midpoint or endpoint impact). Land-use models, in contrast, are top-down. This means that they are based on empirical observations of the state of the environment, but there is no evidence of the connection between the consequence and the (supposed) cause. For example, methods to evaluate biodiversity damage are based on databases of the species present under different land-use types. The reduction in species richness from e.g. a forest to an arable intensive agricultural land is driven by many reasons that partially add to each other: cut down of trees and replacement for crops, use of tractor and other agricultural machinery, emission of pesticides and fertilisers, etc. However, how and how much each of the reasons above contributes to the actual biodiversity loss observed in the agricultural land is not known. The development of mechanistic models such as the ones used to characterise emissions, have the potential to resolve the issue of double counting. For further details see Chap. 40 and Hauschild and Huijbregts (2015).

\subsection{Water Use}

\subsubsection{Problem}

Water is a renewable resource which, thanks to the water cycle, does not disappear. It is a resource different from any other for two main reasons: (1) it is essential for human andecosystem life and (2) its functions are directly linked to its geographic and seasonal availability, since transporting it (and to a lesser extent, storing it) is often impractical and costly. There is sufficient water on our planet to meet current needs of ecosystems and humans. About $119,000 \mathrm{~km}^{3}$ are received every year on land in different forms of precipitation, out of which $62 \%$ are sent back directly to the atmosphere via evaporation and plant transpiration. Out of the $38 \%$ remaining, humans use only about 3\%, out of which $2.1 \%$ for agriculture, $0.6 \%$ for industrial uses and $0.3 \%$ for domestic uses. However, despite these small fractions, there are still important issues associated with water availability. Many important rivers are running dry/from overuse (including the Colorado, Yellow and Indus), greatly affecting local aquatic and terrestrial ecosystems. Humans compete for the use of water in some regions, sometimes leading to the exchange of water rights on the 
market or to the exacerbation of tensions between nations. The World Water Council described the problem well by stating: "There is a water crisis today. But the crisis is not about having too little water to satisfy our needs. It is a crisis of managing water so badly that billions of people - and the environment - suffer badly". In addition to the current mismanagement of the water, which is strongly linked to a competing demand for human uses and ecosystems for a limited renewable resource, the human demand is only increasing, namely due to a growing population and changing diets (with increasing meat consumption). Water availability is also changing due to climate change, aggravating droughts and flooding and hence further increasing the gap between the demand and availability in many highly populated regions around the world. Since the problems associated with water are dependent on where and when water is available, as well as in which quality, it is these aspects that also need to be considered when we assess potential impacts of human freshwater use on the environment (including human health) in LCA.

\subsubsection{Environmental Mechanism}

Before diving into the assessment of potential impacts associated with water, some concepts are important to establish first.

- Types of water use: Water can be used in many different manners and the term water use represents a generic term encompassing any type of use. Consumptive and degradative use are the two main types of use and all other types of use (borrowing, turbinated, cooling, etc.) can generally be defined by one or a combination of the following three terms:

- Water withdrawal: "anthropogenic removal of water from any water body or from any drainage basin either permanently or temporarily" (ISO 2014)

- Consumptive use/water consumption: water use where water is evaporated, integrated in a product or released in a different location then the source

- Degradative use/water degradation: Water that is withdrawn and released in the same location, but with a degraded quality. This includes all forms of pollution: organic, inorganic, thermal, etc. (ISO 2014)

- Sources of water: Different sources of water should be distinguished as impacts from using them will often differ. In general, the following main sources are differentiated: surface water, groundwater, rainwater, wastewater and sea water. Some more specific descriptions can include brackish water (saline water with lower salinity than sea water, generally between 1000 and 10,000 mg/l) or fossil water (non-renewable groundwater)

- Water aváilability: when used as an indicator, this describes the "extent to which humans and ecosystems have sufficient water resources for their needs", with a note that "Water quality can also influence availability, e.g. if quality is not 
sufficient to meet users' needs. If water availability only considers water quantity, it is called water scarcity". (ISO 2014). However, this term is also used to refer to the renewable water volume that is available in a specific area during a specific time, most typically annually or monthly over a watershed $\left(\mathrm{m}^{3} /\right.$ year or $\mathrm{m}^{3} / \mathrm{month}$ )

- Water Scarcity: Different definitions exist for water scarcity, but in LCA the following standardised one is retained: "extent to which demand for water compares to the replenishment of water in an area, e.g. a drainage basin, without taking into account the water quality" (ISO 2014)

- Watershed (also called drainage basin): "Area from which direct surface runoff from precipitation drains by gravity into a stream or other water body" (ISO 2014). In general the main watershed is taken as the reference geographical area to define the same location, as countries are often too large to represent local water issues and smaller areas would lack data and relevance

As mentioned above, freshwater is received from precipitation and a fraction of it (about 38\%) is made available as "blue water", or flowing water which can be used by humans and ecosystems via lakes, rivers or groundwater. Some freshwater is also present in deep fossil aquifers, which are not renewable (not recharged by precipitation), and can be used by humans if pumped out. Groundwater aquifers can recharge lakes and rivers, and vice versa, depending on the topology, soil porosity, etc. Surface water is used by humans, aquatic ecosystems and terrestrial ecosystems, whereas groundwater can be used by some terrestrial ecosystems and humans.

Water use impact assessment at midpoint level typically focuses on water deprivation. Although water is renewed, there is a limited amount available in an area at any point in time, and different users must share, or compete for, the resource. Consuming a certain volume of water will lower its availability for users downstream and may also affect groundwater recharge for example. Users depending on this water may be deprived and suffer consequences. The extent to which they will be deprived will depend on the water scarcity in a region (Fig. 10.25). The higher the demand in comparison to the availability, the more likely a user will be deprived. This user can be (1) humans (present and future

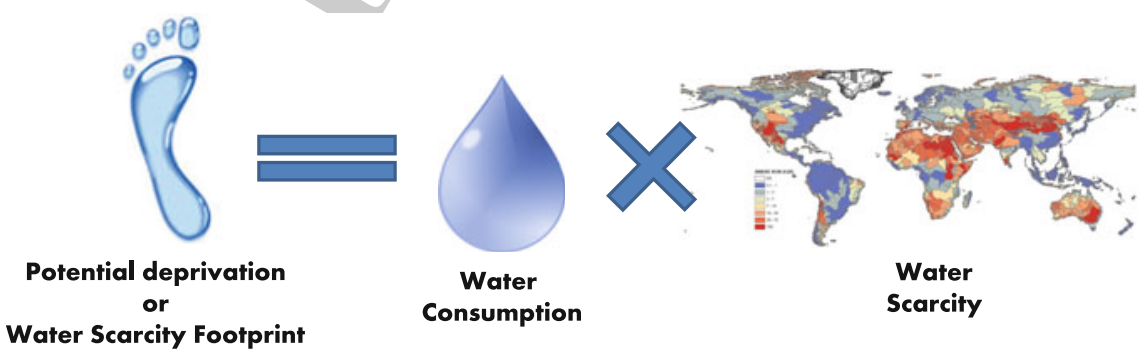

Fig. 10.25 The potential deprivation caused by an additional water consumption in a region is assessed by multiplying this water consumption with a local water scarcity factor. The result is also called a water scarcity footprint 
generations) and (2) ecosystems (Bayart et al. 2010). Quantifying "the potential of a user to be deprived when water is consumed in a region" (Boulay et al. 2017) is the question normally answered at the midpoint level using for example a scarcity indicator (or user-specific deprivation potential if they exist), whereas assessing the potential damages from this deprivation on human health and ecosystem quality is an endpoint assessment.

At the endpoint level, water use impact assessment is focused on the consequences of the water deprivation for humans and ecosystems. The higher the scarcity (and competition between human users), the larger the fraction of an additional water consumption that will deprive another user. Which human user is affected will depend on the share of each water user in a region, as well as their ability to adapt to water deprivation. If the deprived users have access to sufficient socio-economic resources, they may adapt and turn towards a backup technology like desalinisation of seawater or freshwater import to meet their needs. Impacts from human deprivation are then shifted from being solely on human health to all impact categories that are affected by the use of this backup technology. However, if socio-economic means are not sufficient to adapt to lower water and/or food availability, deprivation may occur. Since the potential impacts associated with water deprivation for humans assessed in LCA are on human health, deprivation of water for domestic use, agriculture and aquaculture/fisheries are relevant. Domestic users which already compete for water and have no means to compensate lower water availability via purchasing or technological means will suffer from freshwater deprivation, which is associated to water-related diseases caused by the use of improper water sources and change of behaviour. Agricultural users that are deprived of water for irrigation may produce less, which in turn will lead to lower food availability, either locally or internationally through trade, which may increase health damages associated with malnutrition. Similarly, lower freshwater availability for aquaculture or fisheries could lower fish supply and also contribute to malnutrition impacts, although this was shown to be negligible in comparison to other users' deprivation. This impact pathway, leading to damages on human health, is shown in Fig. 10.26.

Consuming water can also affect water availability for aquatic and terrestrial ecosystems. If the flow of the river is altered, or the volume of the lake is reduced, aquatic ecosystems have less habitat space and may either have to adapt or suffer a change in species density. Since water compartments are strongly interconnected, consuming water in a lake can affect the groundwater availability and vice versa, and each change in availability can lead to a loss of species. Consuming water can also alter the quality by reducing the depth of the water body for example, increasing temperature or concentrating contaminants. Aquatic ecosystems are dependent not only on a minimum volume for their habitat, but also on the flow variations which are naturally influenced by seasons. Human interference with this flow variation can also cause potential species loss. The groundwater table in some regions directly feeds the roots of the vegetation and lowering the aquifer's level can mean that shorter roots species no longer reach their source of water. The relevant mechanisms are summarised in Fig. 10.27. These impact pathways appear 


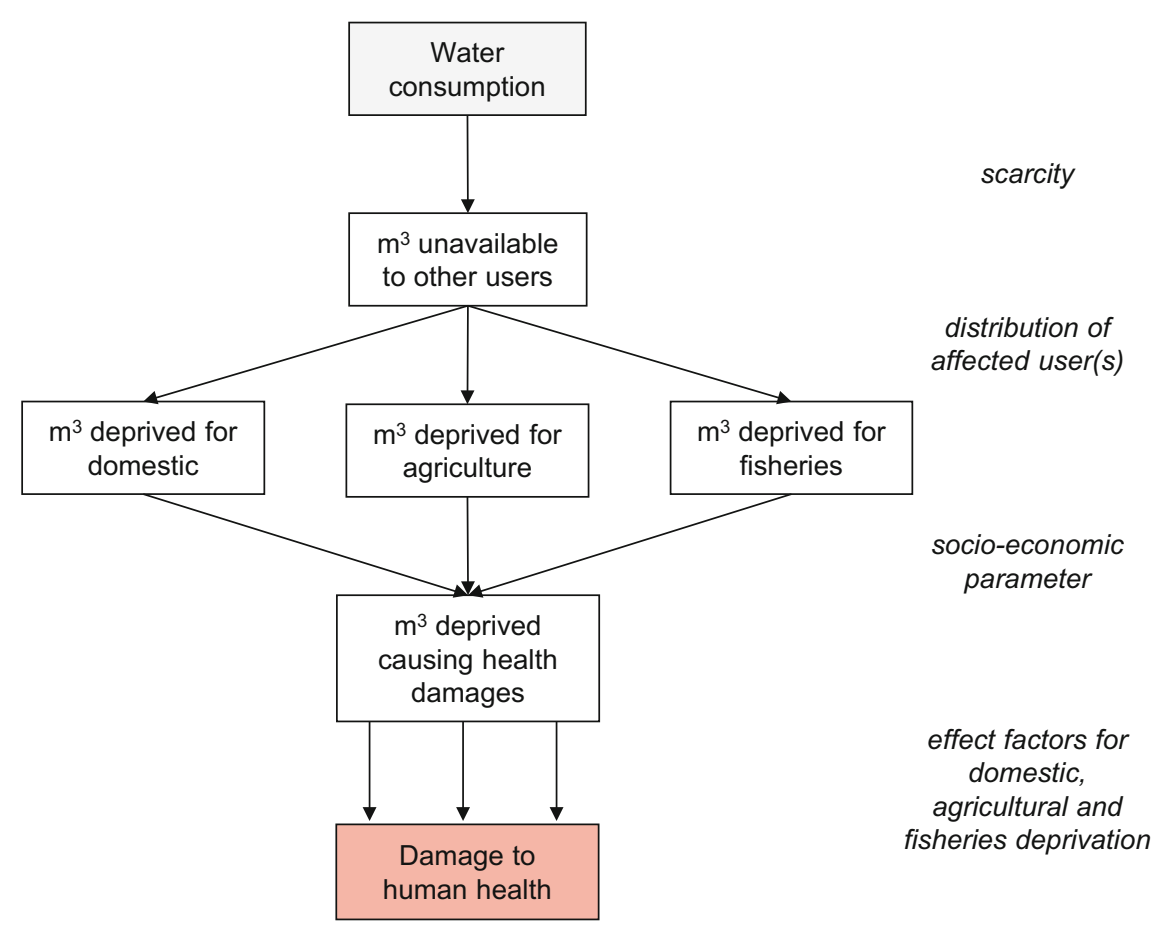

Fig. 10.26 Impact pathway from water consumption to water deprivation for human users leading to potential impacts on human health in Disability Adjusted Life Years (DALY) [adapted from Boulay et al. (2015)]

to be complementary, however more research is needed to determine how they should be used together and to provide one harmonised methodology.

\subsubsection{Existing Characterisation Models}

A stress/scarcity index (here used interchangeably) is the most commonly used midpoint, even if it does not necessarily represent an actual point on the impact pathway of all endpoint categories. A scarcity index is based on the comparison between water used and renewable water available, and represents the level of competition present between the different users (ideally human users and ecosystems). Early indicators (Frischknecht et al. 2008; Pfister et al. 2009) are based on withdrawal-to-availability (WTA) ratios as these were the data available at the time. Since water that is withdrawn but released into the same watershed (within a reasonable time-frame) does not contribute to scarcity, indicators emerged which were based on consumption-to-availability (CTA) ratios instead of withdrawals, when the needed data became available (Boulay et al. 2011; Hoekstra et al. 2012; 


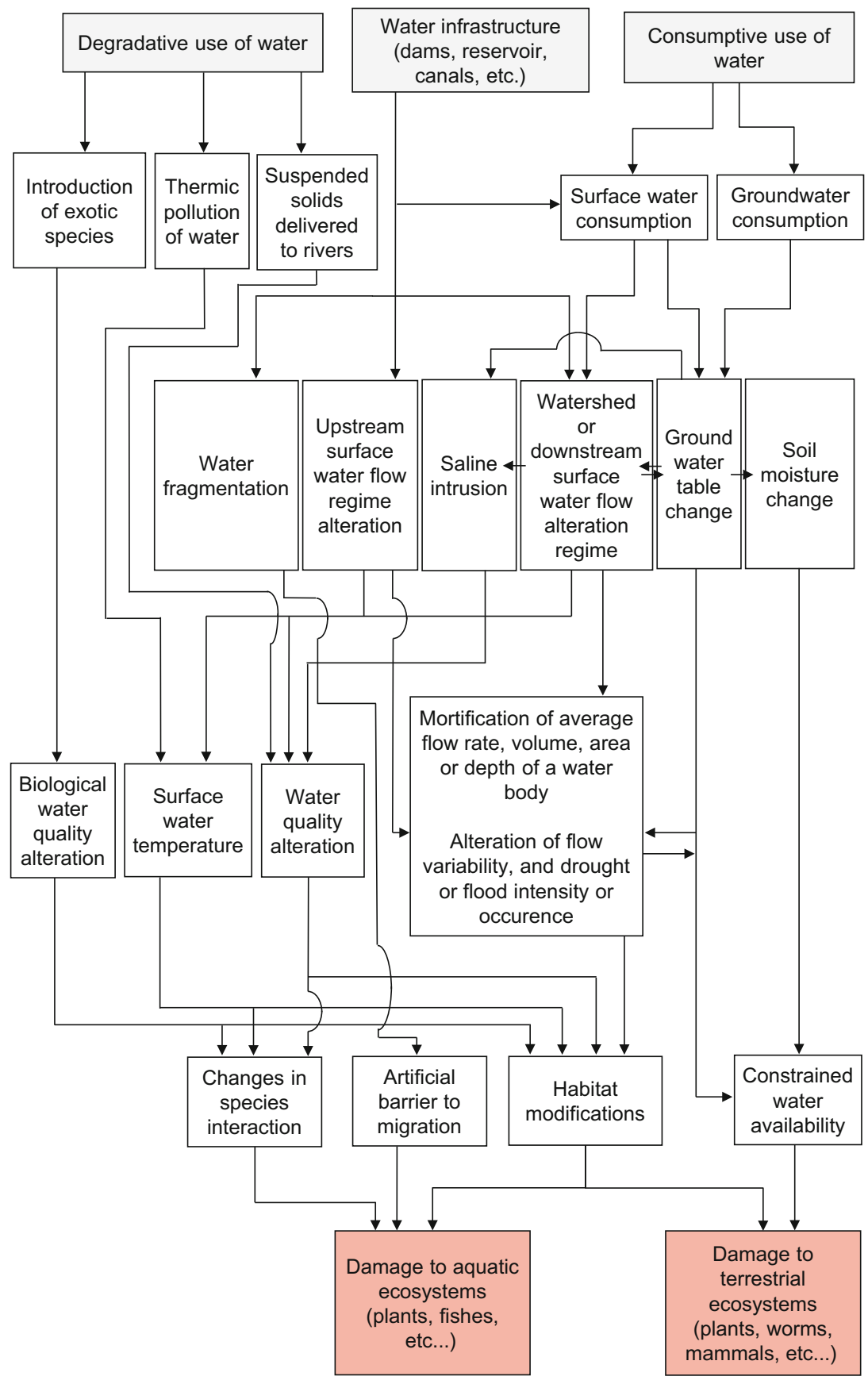

Fig. 10.27 Impact pathway affecting ecosystem quality methods [adapted from Núñez et al. (2016)] 
Berger et al. 2014). Further development led to the inclusion of environmental water requirements as part of the water demand in order to better represent the total water demand from all users, including ecosystems, and resulted in a ratio based on demand-to-availability (DTA) being proposed (Boulay et al. 2014).

One important information was lost in all these indicators: the absolute availability. A ratio of 0.5 may indicate that half of the available water is currently withdrawn, consumed or demanded, but it does not inform on the magnitude of this water volume (i.e. is it 1 or $1000 \mathrm{~m}^{3}$ ?). Regions differ largely in terms of absolute water availability (or aridity) and this information should not be discarded by only looking at the fraction of available water that is being used. In 2016, the WULCA group (see below) proposed the area-specific Available Water Remaining indicator (availability minus demand), AWARE, inverted and normalised with the world average (Boulay et al. 2017). Ranging between 0.1 and 100, this index assesses the potential to deprive another user (human or ecosystem) of water, based on the relative amount, comparing to the world average, of water remaining per area once the demand has been met. The more water remaining compared to the average, the lower the potential to deprive another user, and vice versa.

It should be noted that some midpoints also propose to include quality aspects, allowing the quantification of lower availability being caused by both consumptive and degradative use. This is either done through the use of water quality categories and the assessment of their individual scarcity (Boulay et al. 2011), or through a distance-to-target approach, or dilution volume equivalent, in relation to a reference standard (Ridoutt and Pfister 2010; Bayart et al. 2014).

As mentioned above, human water deprivation can cause health damage by depriving three users: domestic, agriculture or aquaculture/fisheries. Domestic deprivation has been assessed in two methods (Motoshita et al. 2010; Boulay et al. 2011) which quantify the impact pathways described above, either mechanistically or statistically. Both provide characterisation factors in DALY $/ \mathrm{m}^{3}$ consumed and the details of the differences between the methods are described in Boulay et al. (2015).

Agricultural deprivation has been assessed in three methods (Pfister et al. 2009; Boulay et al. 2011; Motoshita et al. 2014). Differences are based on the user competition factor (scarcity) used, the underlying sources of data, the parameter upon which to base the capacity of users to adapt to water deprivation or not, the calculation of the effect factor and, most importantly, the inclusion or not of the trade effect, i.e. the ripple effect of lower food production to lower income and importing countries. Analysis of these methods and modelling choices is provided in Boulay et al. (2015) and at time of writing a consensus was built based on these three models and is described in the Pellston Workshop report from Valencia, 2016.

For the damage that water use may cause on ecosystems, several methods exist that attempt to quantify a part of the complex impact pathways between water consumption and loss of species, i.e. ecosystem quality impacts. An overview of these methods was prepared by Núñez et al. (2016) who analysed in details the existing models, assumptions and consistency. The large majority of them have not yet found their way into LCA practice. None of these endpoint models use water 
scarcity as a modelling parameter, and hence scarcity does not represent a "true midpoint" for ecosystem quality.

The assessment of impacts on the impact category resources, or ecosystem services and resources, is still subject to debate and development. The main question pending being "what exactly are we trying to quantify?". For the case of water, this can be answered in different ways: future generation deprivation, resource-equivalent approach or monetarisation, but these still require further development. The use of non-renewable sources of water fromfossil aquifers would fall in this category.

For further details see Chap. 40 and Hauschild and Huijbregts (2015). Water is a precious resource for humans and ecosystems and our attempts to protect it come in different forms and from different angles. Numerous initiatives exist and indicators of all kinds are emerging regularly and, for the time being, continuously evolving. This should not be perceived as a problem or a sign of lesser value for these indicators; it simply reflects the fact that potential issues associated with water are diverse and so are the approaches to quantify and minimise them. The LCA approach aims to quantify potential impacts associated with human activities (a product, a service or an organisation) on specific areas of protection. Water-related indicators developed within the LCA framework are aligned with this goal, and efforts have been made to build consensus on these methodologies. The WULCA (water use in LCA) expert working group of the UNEP-SETAC Life Cycle Initiative has fostered the development and global harmonisation through international consensus of the water-related impact/assessment methods in LCA. For further information on the existing methods, the reader is encouraged to explore the website: www.wulca-waterlca.org.

\subsection{Abiotic Resource Use}

\subsubsection{Problem}

Natural resources constitute the material foundation of our societies and economies and, paraphrasing the definition of sustainability by the United Nation's Commission on Environment and Development (the Brundtland Commission), they are as such fundamental for our abilities to fulfil our needs as well as for future generations' possibilities to fulfil their own needs. Since we don't know with any certitude what the needs of future generations for specific resources will be, and in order to respect the principle of sustainability, we have to ensure that the future resource availability is as good as possible compared to the current generation's situation, i.e. we have to consider the future availability for all resources that we know and dispose of today.

The definition of natural resources has an anthropocentric starting point. What humans need from nature in order to sustain their livelihood and activities is a 
resource. For the context of LCA, Udo de Haes et al. (1999) thus define natural resources as: “ $\ldots$ those elements that are extracted for human use. They comprise both abiotic resources, such as fossil fuels and mineral ores, and biotic resources, such as wood and fish. They have predominantly a functional value for society."

Although water and land are also resources, their use causes direct impacts on the environment. In this respect they differ from the other resources and they are therefore treated as individual impact categories and described in separate sections. Currently, the resource use impact category covers mostly fossil fuels, minerals and metals so this will also be the focus here.

In terms of future availability of a resource the issue is not the current extraction and use of the resource per se but the depletion or dissipation of the resource. Similar to the use of land, the use of resources can be viewed from an occupation perspective and a transformation perspective. While a resource is used for one purpose it is not available for other purposes, and there is thus a competition situation. When resources are used in a way that caters to their easy reuse at the end of the product life, they are still occupied and not immediately available to other use, but they are in principle available to future use for other purposes. This is the case for many uses of metals today. The occupation perspective is normally not addressed in LCIA of resources today [with the exception of Schneider et al. (2011)]. Rather than resource use the focus of the impact assessment is usually on the resource loss that occurs throughout the life cycle.

Resource loss occurs through transformation of the resource when the use is either consumptive or dispersive. Consumptive resource use converts the resource in a way so that it no longer serves as the resource it was. An example is the use of fossil resources as fuels, converting them in the combustion process into $\mathrm{CO}_{2}$ and water. The transformation occurring in dispersive resource use does not lose the resource but uses it in a way that leads to its dispersal in the technosphere or ecosphere in forms that are less accessible to human use than the original resource was. Dispersive use occurs for most of the metals.

There is still much debate about what the issue of concern of natural resources is and about how this should be addressed in LCIA (Hauschild et al. 2013). This may be explained by the difference in functional values of natural resources on the one hand, and intrinsic or existence values of other impact categories, assessing impacts on human health and ecosystem quality, on the other hand. Steen (2006) summarised different perceptions of the problem with abiotic resources in LCIA as: “... (1) assuming that mining costwill be a limiting factor, (2) assuming that collecting metals or other substances from low-grade sources is mainly an issue of energy, (3) assuming that scarcity is a major threat and (4) assuming that environmental impacts from mining and processing of mineral resources are the main problem."

The extraction of resources and their conversion into materials that are used in product systems are accompanied by energy use and direct emissions that make the raw/material extraction sector an important contributor to environmental impacts and damages in many parts of the world. These impacts are addressed by the other impact categories which are considered in LCA, and hence not treated under the resource depletion impact category. 


\subsubsection{Environmental Mechanism}

With a focus on resource availability for current and future generations, the environmental mechanism may look as shown in Fig. 10.28. It is assumed that resources with easy and/or cheap access and with high concentration or quality are extracted first. Consequently, today's resource extraction will lead future generations to extract lower concentration or lower value resources. This results in additional efforts for the extraction of the same amount of resource which can be translated into higher energy or costs. The endpoint of the impact pathway for resource use is often assessed as the future consequences of resource extraction. Schneider et al. (2014) went further in the pathway with the development of a new model for the assessment of resource provision including economic aspects that influence the security of supply and affect the availability of resources for human use.

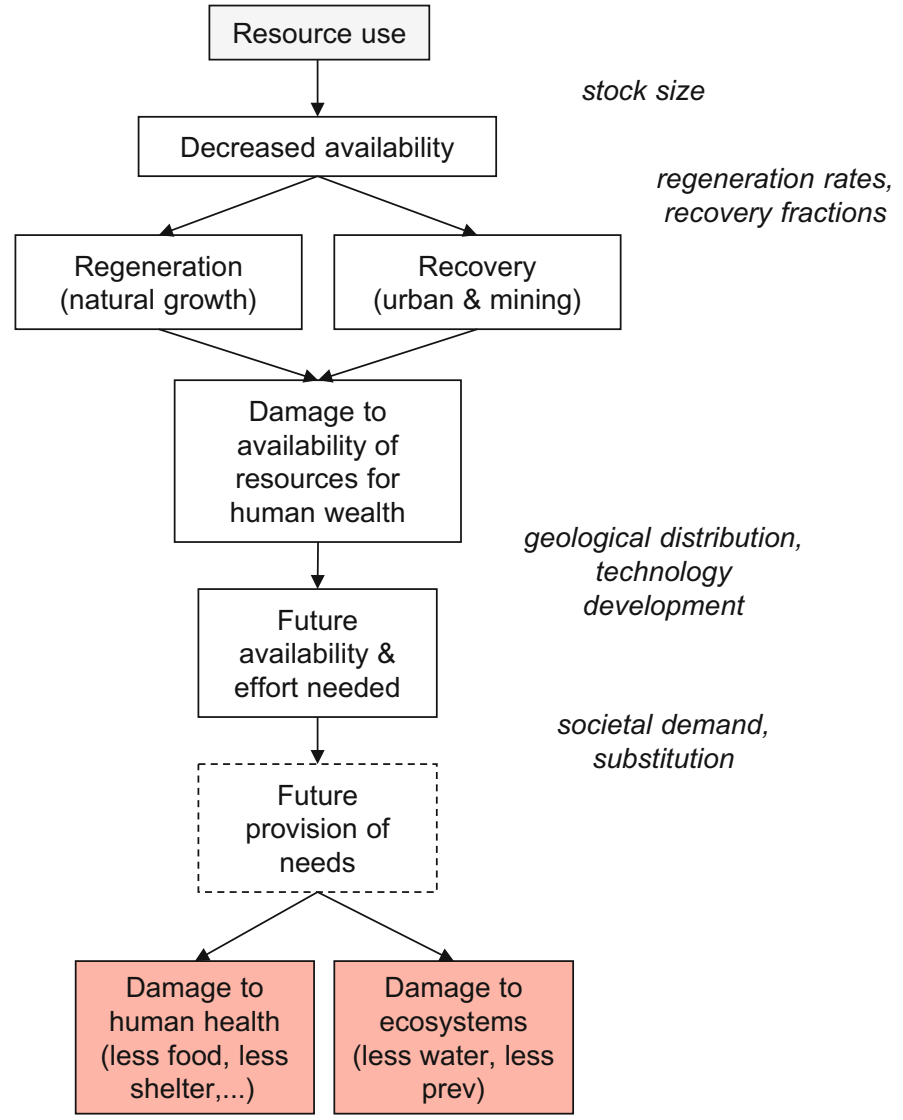

Fig. 10.28 Impact pathway for the resource depletion impact category [adapted from EC-JRC (2010b)] 
Several classification schemesexist for resources (Lindeijer et al. 2002), classifying them according to their origin into Abiotic resources (inorganic materialse.g. water and metals, or organic materials that are non-living at the moment of their extraction - fossil resources) and Biotic resources (living at least until the time of their extraction or harvest from the environment, and hence originating in the biomass). A further classification may be done according to the ability of the resource to be regenerated and the rate by which it may occur. Here resources are classified into:

- Stock resources exist as a finite and fixed amount (reserve) in the ecosphere and are not regenerated (metals in ores) or regenerated so slowly that for practical purposes the regeneration can be ignored (fossil resources)

- Fund resources regenerate but can still be depleted (like the stock resources) if the rate of extraction exceeds the rate of regeneration. Depletion can be temporary if the resource is allowed to recover but it can also be permanent for biotic fund resources where the species underlying the resource becomes extinct. Biotic resources are fund resources but there are also examples of abiotic resources like sand and gravel where the regeneration rate is so high that it is meaningful to classify them as fund resources

- Flow resources are provided as a flow (e.g. solar radiation, wind and to some extent freshwater)and can be harvested as they flow by. Flow resources cannot be globally depleted but there may be local or temporal low availability (notably for freshwater-see Sect. 10.15)

Stock resources are also referred to as non-renewable resources while fund and flow resources jointly are referred to as renewable resources. Resources may also be classified as exhaustible, i.e. they can be completely used up, and inexhaustible, which are unlimited.

\subsubsection{Existing Characterisation Models}

Impacts resulting from resource use are often divided into three categories following the impact pathway (see Fig. 10.28):

1. Methods aggregating natural resource consumption based on an inherent property

2. Methods relating natural resource consumption to resource stocks or availability

3. Methods relating current natural resource consumption to consequences of future extraction of natural resources (e.g. potential increased energy use or costs).

Category 1 methods focus for example on exergy [expressing the maximum amount of useful work the resource can provide in its current form, (Dewulf et al. 2007)], energy (Frischknecht et al. 2015) and solar energy (Rugani et al. 2011). 
While being very reproducible and also easy to determine, the relevance of exergy loss to the scarcity and future availability of the resource is not obvious and therefore these methods are not recommended by the European Commission (EC-JRC 2011). However, the cumulative energy demand (CED) method (Frischknecht et al. 2015) is still used frequently as a resource accounting method in LCA studies and is also part of various comprehensive LCIA methods like CML-IA for fossil fuels (Guinée et al. 2002), ReCiPe (Goedkoop et al. 2012) and the Ecological Scarcity method (Frischknecht and Büsser Knöpfel 2013).

Viewing resource use from a sustainability perspective, the characterisation at midpoint level in the environmental mechanism (Fig. 10.28) should address its impact on the future availability of the resource for human activities. Several category 2 methods do this through incorporating a measure of the scarcity of the resource, expressed by the relationship between what is there and what is extracted, i.e. between the size of the stock or fund and the size of the extraction. However, there are different measures to determine the size of the stock or fund yet to be extracted.

Figure 10.29 shows a terminology for classifying a stock resource into classes according to their economic extractability and whether they are known or unknown. Here we will describe those most used in LCIA. The reserves are the part of the resource which are economically feasible to exploit with current technology. The reserve base is the part of the demonstrated resource that has a reasonable potential to become economically and technically available if the price of the resource increases or if more efficient extraction technology becomes available. Ultimate reserves are the resources that are ultimately available in the earth's crust, which include nonconventional and low-grade materials and common rocks. This reserve

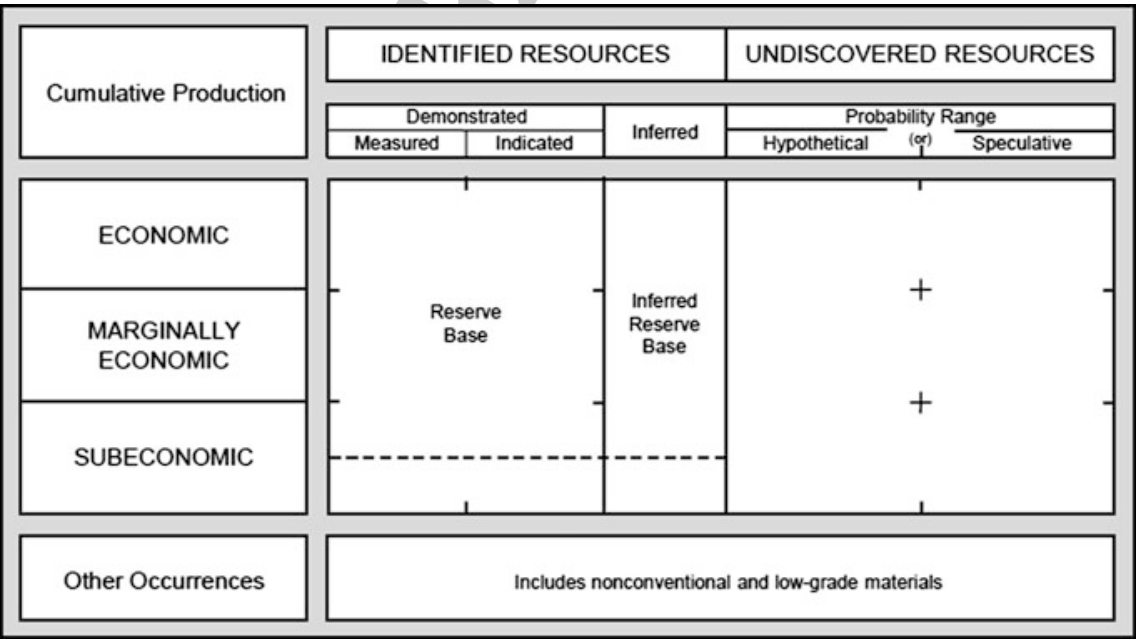

Fig. 10.29 Resource/reserve classification for minerals [taken from U.S. Geological Survey (2015)] 
estimate refers to the quantity of resources that is ultimately available, estimated by multiplying the average natural concentration of the resources in the earth's crust by the mass of the crust. Lately, the extractable geologic resource, also called ultimate recoverable resource and ultimately extractable reserves, has also been adopted by a few LCIA methods. This reserve type is the amount of a given metal in ore in the upper earth's crust that is judged to be extractable over the long term, e.g. $0.01 \%$ (UNEP International Panel on Sustainable Resource Management 2011).

Each reserve estimate has pros and cons. Reserves are known and economically viable to extract, but this amount can fluctuate considerably with changes in prices and discoveries of new deposits. Reserve base has not been reported by the US Geological Survey since 2009 because its size also increases and decreases based on technological advances, economic fluctuations and new discoveries, etc. Consequently, basing the characterisation factoron reserves or reserve base has the problem that it changes with time. Ultimate reserves are calculated on basis of the average concentration of metals in the earth's crust so they are more stable but this is not a good indicator of the quantity of the resource that can realistically be exploited. Finally, the extractable geologic resource seems to be a quite certain reserve estimate but authors are still debating how to quantify it (Schneider et al. 2015).

From the category 2 methods, CML-IA and EDIP are the most widely used. The CML-IA method for characterisation of abiotic stock resources defines an Abiotic Depletion Potential, ADP with a characterisation factor based on the annual extraction rate and the reserve estimates. In Guinée et al. (2002) only the ultimate reserves are included, but Oers et al. (2002) defined additional characterisation factors on the basis of reserves and reserve base estimates. CML-IA using reserve base estimates is the method recommended in the ILCD Handbook for LCIA in the European context (EC-JRC 2011).

An alternative approach inspired by the EDIP method (Hauschild and Wenzel 1998) bases the assessment for the abiotic stock resources on the reserve base and defines the characterisation as the inverse person reserve, i.e. the amount of reserve base per person in the world. For renewable resources, the EDIP inspired characterisation is based on the difference between the extraction rate and the regeneration rate. If the regeneration rate exceeds the extraction rate, it is considered that there is no resource availability issue, and the characterisation factor is given the value 0 .

Further, down the impact pathway, category 3 methods have been developed expressing the future consequences of current resource consumption. Some methods quantify these consequences as additional energy requirements: Eco-Indicator 99, IMPACT 2002+; some methods quantify this effort as additional costs: ReCiPe and Surplus Cost Potential on basis of relationships between extraction and cost increase (Ponsioen et al. 2014; Vieira et al. 2016b), EPS 2000 and the Stepwise method based on willingness to pay; and some methods quantify this effort as additional ore material that has to be dealt with: Ore Requirement Indicator ORI (Swart and Dewulf 2013) and Surplus Ore Potential SOP (Vieira et al. 2016a) used in the LC-IMPACT LCIA method. These methods suffer from a strong dependency on rather uncertain assumptions about the future efficiencies and energy needs of 
mining and extraction technologies, but they seem to better capture the issue of concern which is assuring a supply of resources to future generations.

Schneider et al. (2014) defined a semi-quantitative method expressed as the economic resource scarcity potential (ESP) for evaluating resource use based on life cycle assessment. This method includes elements typically used in the discipline of raw materials criticality, like governance and socio-economic stability, trade barriers, etc., for which each element are scaled to the range $0-1$.

For metal resources, characterisation factors are mostly applied to the metal content in the ore, not the mineral that is extracted. The relevant inventory information is thus the amount of metal used as input, not the amount of mineral. This is also how life cycle inventory (LCI) databases model elementary flows of mineral and metal resources. Schneider et al. (2015) considers not only the geological stock not yet extracted, but also the anthropogenic stock in circulation in products and goods.

The geographic scale at which it is relevant to judge the availability and depletion of a resource depends on the relationship between the price and the density/transportability of the resource. The scale is global for the valuable and dense stock and fund resources that are easy to transport and hence traded on a world market (metals, oil, coal, tropical hardwood), while it is regional for the less valuable and/or less dense stock and fund resources that are used and extracted regionally (natural gas, sand and gravel, limestone) or even locally.

For further details see Chap. 40 and Hauschild and Huijbregts (2015).

\section{References}

Alvarenga, R., Erb, K.-H., Haberl, H., Soares, S., van Zelm, R., Dewulf, J.: Global land use impacts on biomass production - a spatial-differentiated resource-related life cycle impact assessment model. Int. J. Life Cycle Assess. 20, 440-450 (2015)

Apte, J.S., Marshall, J.D., Cohen, A.J., Brauer, M.: Addressing global mortality from ambient PM2.5. Environ. Sci. Technol. 49, 8057-8066 (2015). doi:10.1021/acs.est.5b01236

Bayart, J.-B., Margni, M., Bulle, C., Deschênes, L., Pfister, S., Koehler, A., Vince, F.: Framework for assessment of off-stream freshwater use within LCA. Int. J. Life Cycle Assess. 15, 439-453 (2010)

Bayart, J.-B., Worbe, S., Grimaud, J., Aoustin, E.: The Water Impact Index: a simplified single-indicator approach for water footprinting. Int. J. Life Cycle Assess. 19, 1336-1344 (2014). doi:10.1007/s11367-014-0732-3

Benton, M.J., Twitchet, R.J.: How to kill (almost) all life: the end-Permian extinction event. Trends Ecol. Evol. 18, 358-365 (2003)

Berger, M., van der Ent, R., Eisner, S., Bach, V., Finkbeiner, M.: Water Accounting and Vulnerability Evaluation (WAVE): considering atmospheric evaporation recycling and the risk of freshwater depletion in water footprinting. Environ. Sci. Technol. 48, 4521-4528 (2014). doi:10.1021/es404994t

Bos, U., Horn, R., Beck, T., Lindner, J., Fischer, M.: LANCA ${ }^{\circledR}$ — Characterization Factors for Life Cycle Impact Assessment, Version 2.0. Fraunhofer Verlag, Stuttgart (2016)

Boulay, A.-M., Bulle, C., Bayart, J.-B., Deschenes, L., Margni, M.: Regional characterization of freshwater use in LCA: modeling direct impacts on human health. Environ. Sci. Technol. 45, 8948-8957 (2011) 
Boulay, A.-M., Bare, J., Benini, L., Berger, M., Klemmayer, I., Lathuilliere, M., Loubet, P., Manzardo, A., Margni, M., Ridoutt, B.: Building consensus on a generic water scarcity indicator for LCA-based water footprint: preliminary results from WULCA. LCAFood 2050 (2014)

Boulay, A.-M., Motoshita, M., Pfister, S., Bulle, C., Muñoz, I., Franceschini, H., Margni, M.: Analysis of water use impact assessment methods (part A): evaluation of modeling choices based on a quantitative comparison of scarcity and human health indicators. Int. J. Life Cycle Assess. 20, 139-160 (2015). doi:10.1007/s11367-014-0814-2

Boulay, A.-M., Bare, J., Benini, L., Berger, M., Lathuilliere, M., Manzardo, A., Margni, M., Motoshita, M., Núñez, M., Pastor, A.V., Ridoutt, B.G., Oki, T., Worbe, S., Pfister, S.: The WULCA consensus characterization model for water scarcity footprints: assessing impacts of water consumption based on available water remaining (AWARE) (in review) (2017)

Brandão, M., Milà i Canals, L.: Global characterisation factors to assess land use impacts on biotic production. Int. J. Life Cycle Assess. 18, 1243-1252 (2013). doi:10.1007/s11367-012-0381-3

Brauer, M., Freedman, G., Frostad, J., van Donkelaar, A., Martin, R.V., Dentener, F., van Dingenen, R., Estep, K., Amini, H., Apte, J.S., Balakrishnan, K., Barregard, L., Broday, D., Feigin, V., Ghosh, S., Hopke, P.K., Knibbs, L.D., Kokubo, Y., Liu, Y., Ma, S., Morawska, L., Sangrador, J.L.T., Shaddick, G., Anderson, H.R., Vos, T., Forouzanfar, M.H., Burnett, R.T., Cohen, A.: Ambient air pollution exposure estimation for the global burden of disease 2013 . Environ. Sci. Technol. 50, 79-88 (2016). doi:10.1021/acs.est.5b03709

Chaudhary, A., Verones, F., de Baan, L., Hellweg, S.: Quantifying land use impacts on biodiversity: combining species-area models and vulnerability indicators. Environ. Sci. Technol. 49, 9987-9995 (2015). doi:10.1021/acs.est.5b02507

de Baan, L., Alkemade, R., Koellner, T.: Land use impacts on biodiversity in LCA: a global approach. Int. J. Life Cycle Assess. 18, 1216-1230 (2013a). doi:10.1007/s11367-012-0412-0

de Baan, L., Mutel, C.L., Curran, M., Hellweg, S., Koellner, T.: Land use in life cycle assessment: global characterization factors based on regional and global potential species extinction. Environ. Sci. Technol. 47, 9281-9290 (2013b). doi:10.1021/es400592q

Dewulf, J., De Meester, B., Van der Vorst, G., Van Langenhove, H., Bösch, M., Hellweg, S., Huijbregts, M.A.J.: Cumulative Exergy Extraction from the Natural Environment (CEENE): a comprehensive Life Cycle Impact Assessment method for resource accounting. Environ. Sci. Technol. 41, 8477-8483 (2007)

EC-JRC-European Commission-Joint Research Centre-Institute for Environment and Sustainability: International Reference Life Cycle Data System (ILCD): Handbook-General Guide for Life Cycle Assessment-Detailed Guidance, 1st edn March 2010. EUR 24708 EN. Publications Office of the European Union, Luxembourg (2010a)

EC-JRC-European Commission-Joint Research Centre-Institute for Environment and Sustainability: International Life Cycle Data System (ILCD) Handbook-Framework and Requirements for Life Cycle Impact Assessment Models and Indicators, 1st edn. European Commission, Joint Research Centre, Institute for Environment and Sustainability, Ispra, Italy (2010b)

EC-JRC-European Commission-Joint Research Centre-Institute for Environment and Sustainability: International Reference Life Cycle Data System (ILCD): HandbookRecommendations for Life Cycle Impact Assessment in the European Context-Based on Existing Environmental Impact Assessment Models and Factors, 1st edn 2011, EUR 24571 EN. Publication Office of the European Union, Luxemburg (2011)

EC-JRC: Environmental Footprint-Update of Life Cycle Impact Assessment Methods; DRAFT for TAB (Status: May 2, 2016) Resources, Water, Land. Ispra, Italy (2016)

EEA: The European Environment. State and Outlook 2010. Land Use (2010)

Fantke, P., Jolliet, O., Evans, J.S., Apte, J.S., Cohen, A.J., Hänninen, O.O., Hurley, F., Jantunen, M.J., Jerrett, M., Levy, J.I., Loh, M.M., Marshall, J.D., Miller, B.G., Preiss, P., Spadaro, J.V., Tainio, M., Tuomisto, J.T., Weschler, C.J., McKone, T.E.: Health effects of fine particulate matter in life cycle impact assessment: findings from the Basel Guidance Workshop. Int. J. Life Cycle Assess. 20, 276-288 (2015). doi:10.1007/s11367-014-0822-2 
Foley, J.A., Defries, R., Asner, G.P., Barford, C., Bonan, G., Carpenter, S.R., Chapin, F.S.: Global consequences of land use. Science 309, 570-574 (2005)

Forouzanfar, M.H., Alexander, L., Anderson, H.R., Bachman, V.F., Biryukov, S., Brauer, M., Murray, C.J., et al.: Global, regional, and national comparative risk assessment of 79 behavioural, environmental and occupational, and metabolic risks or clusters of risks in 188 countries, 1990-2013: a systematic analysis for the Global Burden of Disease Study 2013. Lancet 386, 2287-2323 (2015). doi:10.1016/S0140-6736(15)00128-2

Frischknecht, R., Büsser Knöpfel, S.: Swiss eco-factors 2013 according to the ecological scarcity method. In: Methodological Fundamentals and their Application in Switzerland. Environmental Studies No. 1330, Bern, Switzerland (2013)

Frischknecht, R., Steiner, R., Braunschweig, A., Egli, N., Hildesheimer, G.: Swiss Ecological Scarcity Method: The New Version 2006 (2008)

Frischknecht, R., Wyss, F., Büsser Knöpfel, S., Lützkendorf, T., Balouktsi, M.: Cumulative energy demand in LCA: the energy harvested approach. Int. J. Life Cycle Assess. 20, 957-969 (2015). doi:10.1007/s11367-015-0897-4

Goedkoop, M., Heijungs, R., Huijbregts, M.A.J., De Schryver, A., Struijs, J., van Zelm, R., Ministry of Housing SP and E (VROM): ReCiPe 2008-A Life Cycle Impact Assessment Method Which Comprises Harmonised Category Indicators at the Midpoint and the Endpoint Level, 1st edn revised. Ministry of Housing, Spatial Planning and Environment (VROM) (2012)

Gronlund, C., Humbert, S., Shaked, S., O'Neill, M., Jolliet, O.: Characterizing the burden of disease of particulate matter for life cycle impact assessment. Air Qual. Atmos. Health 8, 29-46 (2015). doi:10.1007/s11869-014-0283-6

Guinée, J.B., Gorrée, M., Heijungs, R., Huppes, G., Kleijn, R., van Oers, L., Wegener Sleeswijk, A., Suh, S., Udo de Haes, H.A., de Bruijn, H., van Duin, R., Huijbregts, M.A.J.: Handbook on Life Cycle Assessment: Operational Guide to the ISO Standards. Kluwer Academic Publishers, Dordrecht (2002). ISBN 1-4020-0228-9

Hauschild, M.Z., Huijbregts, M.A.J.: Life cycle impact assessment. In: Klöpffer, W., Curran, M. (eds.) LCA Compend - Complet World Life Cycle Assess, p. 339. Springer, Dordrecht (2015)

Hauschild, M., Wenzel, H.: Environmental Assessment of Products, Volume 2: Scientific Background. Kluwer Academic Publishers, Hingham (1998)

Hauschild, M.Z., Huijbregts, M.A.J., Jolliet, O., MacLeod, M., Margni, M., Van de Meent, D., Rosenbaum, R.K., McKone, T.E.: Building a model based on scientific consensus for life cycle impact assessment of chemicals: the search for harmony and parsimony. Environ. Sci. Technol. 42, 7032-7037 (2008). doi:10.1021/es703145t

Hauschild, M., Goedkoop, M., Guinée, J.B., Heijungs, R., Huijbregts, M., Jolliet, O., Margni, M., Schryver, A., Humbert, S., Laurent, A., Sala, S., Pant, R.: Identifying best existing practice for characterization modeling in life cycle impact assessment. Int. J. Life Cycle Assess. 18, 683697 (2013). doi:10.1007/s11367-012-0489-5

Heijungs, R.: Harmonization of methods for impact assessment. Environ. Sci. Pollut. Res. 2, 217 224 (1995)

Henderson, A., Hauschild, M.Z., Van de Meent, D., Huijbregts, M.A.J., Larsen, H.F., Margni, M., McKone, T.E., Payet, J., Rosenbaum, R.K., Jolliet, O.: USEtox fate and ecotoxicity factors for comparative assessment of toxic emissions in life cycle analysis: sensitivity to key chemical properties. Int. J. Life Cycle Assess. 16, 701-709 (2011). doi:10.1007/s11367-011-0294-6

Hoekstra, A.Y., Mekonnen, M.M., Chapagain, A.K., Mathews, R.E., Richter, B.D.: Global monthly water scarcity: blue water footprints versus blue water availability. PLoS ONE (2012). doi:10.1371/journal.pone.0032688

Hofstetter, P.: Perspectives in Life Cycle Impact Assessment: A Structure Approach to Combine Models of the Technosphere, Ecosphere and Valuesphere. Kluwer Academic Publishers, Dordrecht (1998)

Huijbregts, M.A.J., Hellweg, S., Frischknecht, R., Hungerbühler, K., Hendriks, A.J.: Ecological footprint accounting in the life cycle assessment of products. Ecol. Econ. 64, 798-807 (2008). doi:10.1016/j.ecolecon.2007.04.017 
Huijbregts, M.A.J., Hellweg, S., Hertwich, E.: Do we need a paradigm shift in life cycle impact assessment? Environ. Sci. Technol. 45, 3833-3834 (2011). doi:10.1021/es200918b

Humbert, S., Marshall, J.D., Shaked, S., Spadaro, J.V., Nishioka, Y., Preiss, P., McKone, T.E., Horvath, A., Jolliet, O.: Intake fractions for particulate matter: recommendations for life cycle assessment. Environ. Sci. Technol. 45, 4808-4816 (2011)

IPCC: Climate Change: The IPCC Scientific Assessment. Cambridge University Press, Cambridge (1990)

IPCC: Climate change 2013: the physical science basis. In: Stocker, T.F., Qin, D., Plattner, G.-K., Tignor, M., Allen, S.K., Boschung, J., Nauels, A., Xia, Y. (eds.) Contribution of Working Group I to the Fifth Assessment Report of the Intergovernmental Panel on Climate Change, Cambridge, UK (2013)

IPCC: Climate change 2014: synthesis report. In: Core Writing Team, Pachauri, R.K., Meyer, L.A. (eds.) Contribution of Working Groups I, II and III to the Fifth Assessment Report of the Intergovernmental Panel on Climate Change. IPCC, Geneva (2014a)

IPCC: Climate change 2014: mitigation of climate change. In: Edenhofer, O., Pichs-Madruga, R., Sokona, Y., Farahani, E., Kadner, S., Seyboth, K., Adler, A. (eds.) Contribution of Working Group III to the Fifth Assessment Report of the Intergovernmental Panel on Climate Change, Cambridge, UK (2014b)

ISO: Environmental Management-Life Cycle Assessment-Principles and Framework (ISO 14040). ISO, the International Organization for Standardization, Geneva (2006a)

ISO: Environmental Management-Life Cycle Assessment-Requirements and Guidelines (ISO 14044). ISO, the International Organization for Standardization, Geneva (2006b)

ISO: Environmental Management-Water Footprint-Principles, Requirements and Guidelines (ISO 14046). ISO, the International Organization for Standardization, Geneva (2014)

Koellner, T., Scholz, R.W.: Assessment of land use impacts on the natural environment. Part 1: An analytical framework for pure land occupation and land use change. Int. J. Life Cycle Assess. 12, 16-23 (2007)

Koellner, T., Scholz, R.W.: Assessment of land use impacts on the natural environment. Part 2: Generic characterization factors for local species diversity in Central Europe. Int. J. Life Cycle Assess. 13, 32-48 (2008)

Laurent, A., Hauschild, M.Z.: Impacts of NMVOC emissions on human health in European countries for 2000-2010: use of sector-specific substance profiles. Atmos. Environ. 85, 247 255 (2014). doi:10.1016/j.atmosenv. 2013.11.060

Laurent, A., Olsen, S.I., Hauschild, M.Z.: Limitations of carbon footprint as indicator of environmental sustainability. Environ. Sci. Technol. 46, 4100-4108 (2012). doi:10.1021/ es $204163 \mathrm{f}$

Lenton, T.M., Held, H., Kriegler, E., Hall, J.W., Lucht, W., Rahmstorf, S., Schellnhuber, H.J.: Tipping elements in the Earth's climate system. Proc. Natl. Acad. Sci. 105, 1786-1793 (2008)

Lim, S.S., Vos, T., Flaxman, A.D., Danaei, G., Shibuya, K., Adair-Rohani, H., Memish, Z.A., et al.: A comparative risk assessment of burden of disease and injury attributable to 67 risk factors and risk factor clusters in 21 regions, 1990-2010: a systematic analysis for the Global Burden of Disease Study 2010. Lancet 380, 2224-2260 (2012). doi:10.1016/S0140-6736(12) 61766-8

Lindeijer, E., Müller-Wenk, R., Steen, B.: Impact assessment of resources and land use. In: Udo de Haes, H.A., Finnveden, G., Goedkoop, M., et al. (eds.) Life Cycle Impact Assess. Striving Towar. Best Pract, pp. 11-64. SETAC, Pensacola, USA (2002)

Mackay, D., Seth, R.: The role of mass balance modelling in impact assessment and pollution prevention. In: Sikdar, S.K., Diwekar, U. (eds.) Tools and Methods for Pollution Prevention, pp. 157-179. Kluwer Academic Publishers, Dordrecht (1999)

Mila i Canals, L., de Baan, L.: Land use. In: Hauschild, M.Z., Huijbregts, M.A.J. (eds.) Chapter 11: Life cycle impact assessment. LCA Compend.-Complet. World Life Cycle Assessment, Life Cycle Impact Assess, pp 197-222. Springer, Dordrecht (2015)

Millenium Ecosystem Assessment: Ecosystems and human well-being: biodiversity Synthesis. World Resources Institute, Washington, DC (2005) 
Motoshita, M., Itsubo, N., Inaba, A.: Development of impact factors on damage to health by infectious diseases caused by domestic water scarcity. Int. J. Life Cycle Assess. 16, 65-73 (2010)

Motoshita, M., Ono, Y., Pfister, S., Boulay, A.-M., Berger, M., Nansai, K., Tahara, K., Itsubo, N., Inaba, A.: Consistent characterisation factors at midpoint and endpoint relevant to agricultural water scarcity arising from freshwater consumption. Int. J. Life Cycle Assess. (2014). doi:10. 1007/s11367-014-0811-5

Müller-Wenk, R., Brandão, M.: Climatic impact of land use in LCA — carbon transfers between vegetation/soil and air. Int. J. Life Cycle Assess. 15, 172-182 (2010)

Murray, C.J., Lopez, A.D.: The Global Burden of Disease. Harvard School of Public Health, World Bank, and World Health Organization, Geneva, Switzerland (1996)

Nilsson, J., Grennfelt, P.: Critical loads for sulphur and nitrogen. Report from a Workshop held at Skokloster, Sweden, 19-24 March 1988. Environmental Report 1988:15, Copenhagen, Denmark (1988)

Núñez, M., Civit, B., Muñoz, P., Arena, A.P., Rieradevall, J., Antón, A.: Assessing potential desertification environmental impact in life cycle assessment. Part 1: methodological aspects. Int. J. Life Cycle Assess. 15, 67-78 (2010)

Núñez, M., Antón, A., Muñoz, P., Rieradevall, J.: Inclusion of soil erosion impacts in life cycle assessment on a global scale: application to energy crops in Spain. Int. J. Life Cycle Assess. 18, 755-767 (2013). doi:10.1007/s11367-012-0525-5

Núñez, M., Bouchard, C., Bulle, C., Boulay, A.-M., Margni, M.: Critical analysis of life cycle impact assessment methods addressing consequences of freshwater use on ecosystems and recommendations for future method development. Int. J. Life Cycle Assess. 21, 1799-1815 (2016). doi:10.1007/s11367-016-1127-4

Pennington, D.W., Rydberg, T., Potting, J., Finnveden, G., Lindeijer, E., Jolliet, O., Rebitzer, G.: Life cycle assessment Part 2: Current impact assessment practice. Environ. Int. 30, 721-739 (2004)

Pfister, S., Koehler, A., Hellweg, S.: Assessing the environmental impacts of freshwater consumption in LCA. Environ. Sci. Technol. 43, 4098-4104 (2009)

Ponsioen, T.C., Vieira, M.D.M., Goedkoop, M.J.: Surplus cost as a life cycle impact indicator for fossil resource scarcity. Int. J. Life Cycle Assess. 19, 872-881 (2014). doi:10.1007/s11367013-0676-Z

Rees, W.E.: Ecological footprints and appropriated carrying capacity: what urban economics leaves out. Environ. Urban 4, 121-130 (1992). doi:10.1177/095624789200400212

Ridoutt, B.G., Pfister, S.: A revised approach to water footprinting to make transparent the impacts of consumption and production on global freshwater scarcity. Glob. Environ. Change 20, 113120 (2010)

Ridoutt, B.G., Fantke, P., Pfister, S., Bare, J., Boulay, A.-M., Cherubini, F., Frischknecht, R., Hauschild, M., Hellweg, S., Henderson, A., Jolliet, O., Levasseur, A., Margni, M., McKone, T., Michelsen, O., Milà i Canals, L., Page, G., Pant, R., Raugei, M., Sala, S., Saouter, E., Verones, F., Wiedmann, T.: Making sense of the minefield of footprint indicators. Environ. Sci. Technol. 49, 2601-2603 (2015). doi:10.1021/acs.est.5b00163

Ridoutt, B.G., Pfister, S., Manzardo, A., Bare, J., Boulay, A.-M., Cherubini, F., Fantke, P., Frischknecht, R., Hauschild, M., Henderson, A., Jolliet, O., Levasseur, A., Margni, M., McKone, T., Michelsen, O., i Canals, L., Page, G., Pant, R., Raugei, M., Sala, S., Verones, F.: Area of concern: a new paradigm in life cycle assessment for the development of footprint metrics. Int. J. Life Cycle Assess. 21, 276-280 (2016). doi:10.1007/s11367-015-1011-7

Rosenbaum, R.: Selection of impact categories, category indicators and characterization models in goal and scope definition. In: Curran, M.A. (ed.) Chapter 2: Goal and Scope Definition in Life Cycle Assessment. LCA Compend.-Complet. World Life Cycle Assess (2016)

Rosenbaum, R.K., Bachmann, T.M.K., Gold, L.S., Huijbregts, M.A.J., Jolliet, O., Juraske, R., Koehler, A., Larsen, H.F., MacLeod, M., Margni, M., McKone, T.E., Payet, J., Schuhmacher, M., Van de Meent, D., Hauschild, M.Z.: USEtox - The UNEP/SETAC-consensus model: recommended characterisation factors for human toxicity and freshwater ecotoxicity in Life 
Cycle Impact Assessment. Int. J. Life Cycle Assess. 13, 532-546 (2008). doi:10.1007/s11367008-0038-4

Rugani, B., Huijbregts, M.A.J., Mutel, C., Bastianoni, S., Hellweg, S.: Solar energy demand (SED) of commodity life cycles. Environ. Sci. Technol. 45, 5426-5433 (2011).doi:10.1021/ es103537f

Saad, R., Koellner, T., Margni, M.: Land use impacts on freshwater regulation, erosion regulation, and water purification: a spatial approach for a global scale level. Int. J. Life Cycle Assess. 18, 1253-1264 (2013). doi:10.1007/s11367-013-0577-1

Safire, W.: Footprint. New York Times Sunday Mag MM20 (2008)

Salisbury, F.B., Ross, C.W.: Plant Physiology, 3rd edn. Wadsworth Publishing Company, Belmont (1978)

Schneider, L., Berger, M., Finkbeiner, M.: The anthropogenic stock extended abiotic depletion potential (AADP) as a new parameterisation to model the depletion of abiotic resources. Int. J. Life Cycle Assess. 16, 929-936 (2011). doi:10.1007/s11367-011-0313-7

Schneider, L., Berger, M., Schüler-Hainsch, E., Knöfel, S., Ruhland, K., Mosig, J., Bach, V., Finkbeiner, M.: The economic resource scarcity potential (ESP) for evaluating resource use based on life cycle assessment. Int. J. Life Cycle Assess. 19, 601-610 (2014). doi:10.1007/ s11367-013-0666-1

Schneider, L., Berger, M., Finkbeiner, M.: Abiotic resource depletion in LCA—background and update of the anthropogenic stock extended abiotic depletion potential (AADP) model. Int. J. Life Cycle Assess. 20, 709-721 (2015). doi:10.1007/s11367-015-0864-0

Steen, B.A.: Abiotic resource depletion different perceptions of the problem with mineral deposits. Int. J. Life Cycle Assess. 11, 49-54 (2006). doi:10.1065/lca2006.04.011

Stumm, W., Morgan, J.J.: Aquatic chemistry-introduction emphasizing chemical equilibria in natural waters, 2nd edn. Wiley, New York (1981)

Swart, P., Dewulf, J.: Quantifying the impacts of primary metal resource use in life cycle assessment based on recent mining data. Resour. Conserv. Recycl. 73, 180-187 (2013). doi:10. 1016/j.resconrec.2013.02.007

Thompson, M., Ellis, R.J., Wildavsky, A.: Cultural Theory. Westview Press, Boulder (1990)

Udo de Haes, H.A., Jolliet, O., Finnveden, G., Hauschild, M., Krewitt, W., Mueller-Wenk, R.: Best available practice regarding impact categories and category indicators in life cycle impact assessment. Part 1. Int. J. Life Cycle Assess. 4, 167-174 (1999)

UNEP International Panel on Sustainable Resource Management: Estimating long-run geological stocks of metals (2011)

U.S. Geological Survey: Mineral Commodity Summaries 2015: U.S. Geological Survey (2015)

Van Oers, L., De Koning, A., Guinée, J., Huppes, G.: Abiotic Resource Depletion in LCAImproving Characterization Factors for Abiotic Resource Depletion as Recommended in the New Dutch LCA Handbook. Road and Hydraulic Engineering Institute of the Dutch Ministry of Transport, Public Works and Water Management (V\&W), Delft, The Netherlands (2002)

van Zelm, R., Huijbregts, M.A.J,, den Hollander, H.A., van Jaarsveld, H.A., Sauter, F.J., Struijs, J., van Wijnen, H.J., van de Meent, D.: European characterization factors for human health damage of PM10 and ozone in life cycle impact assessment. Atmos. Environ. 42, 441-453 (2008). doi:10.1016/j.atmosenv.2007.09.072

van Zelm, R., Preiss, P., van Goethem, T., Van Dingenen, R., Huijbregts, M.: Regionalized life cycle impact assessment of air pollution on the global scale: damage to human health and vegetation. Atmos. Environ. 134, 129-137 (2016). doi:10.1016/j.atmosenv.2016.03.044

Vieira, M.D.M., Ponsioen, T.C., Goedkoop, M.J., Huijbregts, M.A.J.: Surplus ore potential as a scarcity indicator for resource extraction. J. Ind. Ecol. (2016a). doi:10.1111/jiec.12444

Vieira, M.D.M., Ponsioen, T.C., Goedkoop, M.J., Huijbregts, M.A.J.: Surplus cost potential as a life cycle impact indicator for metal extraction. Resources 5, 2 (2016b). doi:10.3390/ resources 5010002

WHO: Indoor air quality: organic pollutants. Report on a WHO Meeting, Berlin, 23-27 August 1987. EURO Reports and Studies 111. Geneva, Switzerland (1989) 
WHO: Health risks of particulate matter from long-range transboundary air pollution, Bonn, Germany (2006)

WMO: Scientific Assessment of Ozone Depletion: 2014, World Meteorological Organization, Global Ozone Research and Monitoring Project Report No. 55. Geneva, Switzerland (2014)

\section{Author Biographies}

Ralph K. Rosenbaum LCA expert and environmental modeller focusing on LCIA development since early 2000s. Contributed to several UNEP/SETAC working groups towards global harmonisation of LCA methodology. Interested in LCIA modelling of emissions and water/soil resource use, operationalisation of uncertainty management and spatial differentiation.

Michael Hauschild Involved in development of LCIA methodology since the early 1990s. Has led several SETAC and UNEP/SETAC working groups and participated in the development of the ISO standards and the ILCD methodological guidelines. Main LCA interests are chemical impacts, spatial differentiation and science-based boundaries in LCIA.

Anne-Marie Boulay LCA and water footprint expert focusing on water use impacts assessment in LCA since 2008. Chaired UNEP/SETAC and FAO working groups towards harmonisation of water use impact assessment methodologies, and has been involved in ISO standards development on water footprint, providing training on the topic for UNEP and ISO worldwide.

Peter Fantke Develops methods for LCIA, health impact assessment and chemical alternatives assessment since 2006. Has contributed to UNEP/SETAC LCIA working groups and is USEtox Manager. Interested in quantifying and characterising chemical emissions, uncertainty analysis, consumer exposure, chemical substitution and model parameterisation.

Alexis Laurent Working with LCA since 2010 with a strong LCIA focus, particularly on normalisation aspects. Main LCA interests include development of LCIA methods, LCA applications and footprinting of large-scale systems for policy-making (e.g. nations, sectors), and LCA applied to various technology domains, including energy systems.

Montserrat Núñez Environmental scientist with interest in LCA, agriculture, and modelling of environmental impacts from resource use in agricultural activities. Involved in development of LCIA methods to assess impacts of land use and water use since 2007.

Marisa Vieira Both as researcher and consultant developing and applying LCA, LCM and footprints since 2007. Main interests are the application of life cycle thinking in companies, branch associations and policy and capability development through training on LCA, SimaPro and Environmental Footprint. 